ESAIM: M2AN 50 (2016) 1425-1455

DOI: $10.1051 / \mathrm{m} 2 \mathrm{an} / 2015080$
ESAIM: Mathematical Modelling and Numerical Analysis

www.esaim-m2an.org

\title{
MULTISCALE ANALYSIS OF LINEAR EVOLUTION EQUATIONS WITH APPLICATIONS TO NONLOCAL MODELS FOR HETEROGENEOUS MEDIA $*, * *, * * *$
}

\author{
Qiang Du ${ }^{1}$, Robert Lipton ${ }^{2}$ and Tadele Mengesha ${ }^{3}$
}

\begin{abstract}
The method of two scale convergence is implemented to study the homogenization of time-dependent nonlocal continuum models of heterogeneous media. Two integro-differential models are considered: the nonlocal convection-diffusion equation and the state-based peridynamic model in nonlocal continuum mechanics. The asymptotic analysis delivers both homogenized dynamics as well as strong approximations expressed in terms of a suitable corrector theory. The method provides a natural analog to that for the time-dependent local PDE models with highly oscillatory coefficients with the distinction that the driving operators considered in this work are bounded.
\end{abstract}

Mathematics Subject Classification. 74Q05, 74E05, 74H10, 45F99, 45P05.

Received January 17, 2014. Revised February 20, 2015. Accepted September 14, 2015.

\section{INTRODUCTION}

A popular approach to model and analyze the effect of heterogeneities across macroscopic length scales is given by the theory of homogenization. This approach is now highly developed for continuum models described by partial differential equations. Recently, studies of nonlocal models have attracted much attention. For example, for the mechanics of materials deformation and failure, a nonlocal peridynamic (PD) continuum theory has been introduced by Silling in [31] and subsequently extended in [32,33].

The PD formulation is distinguished in its ability to seamlessly evolve both smooth and discontinuous deformation in a way useful for describing propagation of singularities. Peridynamic modeling in the presence of heterogeneities is challenging; however recent work offers proposals for the modeling and simulation of composite

Keywords and phrases. Multiscale analysis, peridynamics, nonlocal equations, Navier equation, homogenization, heterogeneous materials, two-scale convergence.

* Qiang Du's research is supported in part by NSF grant DMS-1318586, DMS-1312809 and AFOSR MURI center for Material Failure Prediction through peridynamics.

** Robert Lipton's research is supported in part by NSF grant DMS-1211066 and NSF EPSCOR Cooperative Agreement No. EPS-1003897 with additional support from the Louisiana Board of Regents.

*** Tadele Mengesha's research is supported by NSF grant DMS-1506512.

1 Department of Applied Physics and Applied Mathematics, Columbia University, New York, 10027, USA. qd2125@columbia.edu

2 Department of Mathematics, Center for Computation and Technology, Louisiana State University, Baton Rouge, LA, 70803, USA. lipton@math.1su.edu

3 Department of Mathematics, The University of Tennessee, Knoxville, TN 37996, USA. mengesha@utk.edu 
material systems using peridynamics $[6,7,18,19,21]$. These approaches are of the top down type and are based on a volume averaged approach so that forces acting across macroscopic length scales are spatial averages of forces acting across smaller length scales. A more explicit account of heterogeneities has also been considered within the peridynamic model at the computational meshing level, see for example [21]. On the other hand, in the peridynamics framework, bottom up multiscale approaches are given in [2,25]. Here local field interaction between heterogeneities are modeled directly. The work in [2] provides a systematic and rigorous method for developing multiscale approximations to solutions of bond based peridynamic problems in fiber reinforced media. The approach delivers homogenized dynamics and strong approximation using correctors. A similar but mathematically distinct analysis for the stationary case is provided in [25] for the more general time-independent state-based model. In contrast to the latter, the present work is focused on time-dependent model problems.

Besides the nonlocal peridynamic theory, there are also other nonlocal evolution equations of practical interests. The so called nonlocal convection-diffusion equations [13] provides nonlocal extensions of the classical convection-diffusion equations. It can be used to describe general stochastic jump processes. For heterogeneous media, we may encounter cases in which the jump rates are spatially dependent and vary on both fast and slow scales. This leads to another example of homogenized dynamics of nonlocal evolution equations.

In this paper, we build on earlier work on local homogenization $[3,9,14,15,27,29,34]$ and develop a framework for identifying the homogenized evolution and corrector theory for linear evolution equations associated with integral bounded operators defined over a heterogeneous medium. To describe the main results in this paper, let us consider the nonlocal convection-diffusion equation for simplicity. Suppose that $\Omega$ is an open and bounded subset of $\mathbb{R}^{d}$, and $Y$ is the unit cube in $\mathbb{R}^{d}$ for some $d \geq 1$. Given a continuous function $\Theta(\mathbf{x}, \mathbf{y})$ that is periodic in $\mathbf{y}$, and an integrable even function $\rho(\boldsymbol{\xi})$ we consider the nonlocal highly oscillatory operator

$$
\mathcal{L}_{\epsilon} u(\mathbf{x})=\int_{\Omega} \rho\left(\mathbf{x}^{\prime}-\mathbf{x}\right)\left(\Theta\left(\mathbf{x}^{\prime}, \frac{\mathbf{x}^{\prime}}{\epsilon}\right) u\left(\mathbf{x}^{\prime}\right)-\Theta\left(\mathbf{x}, \frac{\mathbf{x}}{\epsilon}\right) u(\mathbf{x})\right) \mathrm{d} \mathbf{x}^{\prime}
$$

and the nonlocal convection-diffusion equation

$$
\left\{\begin{array}{l}
u_{t}^{\epsilon}(\mathbf{x}, t)=\mathcal{L}_{\epsilon} u^{\epsilon}(\mathbf{x}, t), \text { for } \mathbf{x} \in \Omega, t>0 \\
u^{\epsilon}(\mathbf{x}, 0)=u_{0}\left(\mathbf{x}, \frac{\mathbf{x}}{\epsilon}\right), \text { for } \mathbf{x} \in \Omega
\end{array}\right.
$$

for a given sufficiently smooth initial data $u_{0}(\mathbf{x}, \mathbf{y})$. For $1<p<\infty$, the operator $\mathcal{L}_{\epsilon}: L^{p}(\Omega) \rightarrow L^{p}(\Omega)$ is a uniformly bounded (in $\epsilon$ ) linear operator. As a consequence, corresponding to each $\epsilon>0$ and $T>0$ a unique solution $u^{\epsilon} \in C^{1}\left([0, T] ; L^{p}(\Omega)\right)$ exists to (1.1). Moreover, the sequence of solutions $u^{\epsilon}$ is also bounded in $C^{1}\left([0, T] ; L^{p}(\Omega)\right)$. One of the main results of this paper is the qualitative study of the asymptotic behavior of $u^{\epsilon}$ as $\epsilon \rightarrow 0$. It is not surprising that reflecting the presence of high oscillations in the "coefficient" $\Theta(\mathbf{x}, \mathbf{x} / \epsilon)$, the solutions $u^{\epsilon}$ will also have high fluctuations. We will use the notion of two scale convergence to capture the fast and slow scales of fluctuation of $u^{\epsilon}$. To that end, we will prove the following theorem.

Theorem 1.1. The sequence of solutions $u^{\epsilon}$ to (1.1) two scale converges to $u(\mathbf{x}, \mathbf{y}, t)$ in $L^{p}(\Omega \times Y)$, where $u$ solves the two scale evolution equation

$$
\left\{\begin{array}{l}
u_{t}(\mathbf{x}, \mathbf{y}, t)=\mathcal{L}_{0} u(\mathbf{x}, \mathbf{y}, t), \text { for }(\mathbf{x}, \mathbf{y}) \in \Omega \times Y, \quad 0<t<T \\
u(\mathbf{x}, \mathbf{y}, 0)=u_{0}(\mathbf{x}, \mathbf{y}), \text { for }(\mathbf{x}, \mathbf{y}) \in \Omega
\end{array}\right.
$$

Moreover, for each $t \in[0, T], \mathbf{x} \mapsto u\left(\mathbf{x}, \frac{\mathbf{x}}{\epsilon}, t\right)$ gives a strong approximation in the sense that

$$
\lim _{\epsilon \rightarrow 0}\left\|u^{\epsilon}(\cdot, t)-u\left(\mathbf{x}, \frac{\dot{ }}{\epsilon}\right), t\right\|_{L^{p}(\Omega)}=0 .
$$


The two-scale operator $\mathcal{L}_{0}$ presented in the above theorem is given by

$$
\mathcal{L}_{0} \mathbf{u}(\mathbf{x}, \mathbf{y})=\int_{\Omega} \int_{Y} \rho\left(\mathbf{x}^{\prime}-\mathbf{x}\right)\left(\Theta\left(\mathbf{x}^{\prime}, \mathbf{y}^{\prime}\right) u\left(\mathbf{x}^{\prime}, \mathbf{y}^{\prime}\right)-\Theta(\mathbf{x}, \mathbf{y}) u(\mathbf{x}, \mathbf{y})\right) \mathrm{d} \mathbf{x}^{\prime} \mathrm{d} \mathbf{y}^{\prime}
$$

is a bounded linear operator on $L^{p}(\Omega \times Y)$. We notice that from the two scale convergence, $u^{\epsilon}$ actually converges weakly in $L^{p}$ to $u^{H}(\mathbf{x}, t)=\int_{Y} u(\mathbf{x}, \mathbf{y}, t)$ dy making it the effective or homogenized solution. Rewriting $u(\mathbf{x}, \mathbf{y}, t)=$ $u^{H}(\mathbf{x}, t)+w(\mathbf{x}, \mathbf{y}, t)$, the implication of Theorem 1.1 is that $w(\mathbf{x}, \mathbf{x} / \epsilon, t)$ serves as a corrector as the strong approximation result in Theorem 1.1 states that for each $t \in[0, T]$

$$
\left\|u^{\epsilon}(\cdot, t)-u^{H}(\cdot, t)-w(\cdot, \cdot / \epsilon, t)\right\|_{L^{p}} \rightarrow 0, \quad \text { as } \epsilon \rightarrow 0 .
$$

A similar result as Theorem 1.1 will be proven for the heterogeneous peridynamic equation of motion in Section 4 . We note that the results and the approach used have similar structure as in the work in [2]. However this work is distinct from [2] in many technical details. To begin with, the focus in [2] is the implementation of the method of two scale convergence to the study of the homogenization of bond-based peridynamic models. The current work is focused on the more inclusive and technically involved state-based model. The bond-based peridynamic model considered in $[2]$ was also specific and is based on the interaction kernel $\rho(\boldsymbol{\xi})=\frac{1}{|\boldsymbol{\xi}|}$ in $\mathbb{R}^{3}$ (this corresponds to the linearized bond-stretch model given in [31]), whereas in this paper we consider any positive even integral kernel $\rho$, based on the linearized model given in [33]. To handle this major difference, the technical approach implemented is also significantly different. A consequence of this is that, for example, the strong approximation results in [2] is valid for $3 / 2<p<\infty$ and uses the function space $C\left(\Omega ; L_{\mathrm{per}}^{p}(Y)\right)$, while in this work it is valid for all $p \in(1, \infty)$ and uses the function space $L^{p}\left(\Omega ; C_{\text {per }}(Y)\right)$. The implementation of the approach to the convection-diffusion equation is also another distinctive feature of this paper.

In this paper we consider nonlocal multiscale convection diffusion equations with kernels having oscillations over the length $\epsilon$ relative to the horizon size. This choice is made to illustrate how a non-local homogenized operator is recovered in the limit. The analysis of the heterogeneous multiscale equation of motion is carried out in the presence of two length scales of interaction. The small length scale is associated with small inhomogeneities interacting over a microscopic horizon of length $\epsilon$. The larger length scale is fixed and represents nonlocal interaction over a mesoscopic horizon of order unity. Other choices for the scaling of horizon and kernel can be made and this is the subject of future study. See the discussion in Remark 4.1 in Section 4.

The plan of the paper is as follows. The notion of two scale convergence and other main ingredients are reviewed in Section 2. In the same section, we will provide a rundown of the asymptotic analysis program we will be using. In Section 3, the proof of the above theorem is given with proper detail for the non-local linear convection-diffusion equations. Homogenized and corrector evolution equations will be provided for $u^{H}$ and $w$ as coupled systems. The program will also be implemented to the state based peridynamic evolution system in Section 4. In the two model problems, the solution of the original evolution equation is represented by a strong $L^{p}$ approximation of the sum of the homogenized evolution and the corrector that directly encodes the effects of local oscillations in the medium.

\section{TOOLS AND PRELIMINARY RESUlts}

We develop the asymptotic analysis of time-dependent problems of the form

$$
\left\{\begin{array}{l}
\mathbf{u}_{t}^{\epsilon}(\mathbf{x}, t)=\mathcal{P}_{\epsilon} \mathbf{u}^{\epsilon}(\mathbf{x}, t)+\mathbf{b}_{\epsilon}(\mathbf{x}, t), \text { for } \mathbf{x} \in \Omega, t>0 \\
\mathbf{u}^{\epsilon}(\mathbf{x}, 0)=\mathbf{u}_{0}^{\epsilon}(\mathbf{x}), \text { for } \mathbf{x} \in \Omega
\end{array}\right.
$$

where for positive integers $k$ and $d, \Omega \subset \mathbb{R}^{d}$ is an open bounded domain, and $\mathcal{P}_{\epsilon}: L^{p}\left(\Omega ; \mathbb{R}^{k}\right) \rightarrow L^{p}\left(\Omega ; \mathbb{R}^{k}\right)$ is a family of bounded linear operators parametrized by $\epsilon>0$ with the uniform bound

$$
\sup _{\epsilon>0}\left\|\mathcal{P}_{\epsilon}\right\| \leq M<\infty
$$


for some positive constant $M$. Here, the parameter $\epsilon$ characterizes, say in the case of nonlocal diffusion models, the scale of heterogeneities or fine scale oscillations of material properties, and $\|\cdot\|$ denotes the operator norm for operators defined on the space $L^{p}\left(\Omega ; \mathbb{R}^{k}\right)$ with $1<p<\infty$. For notational convenience, we write $\mathbf{u}^{\epsilon}(t)=\mathbf{u}^{\epsilon}(\cdot, t)$, and $\mathbf{b}_{\epsilon}(t)=\mathbf{b}_{\epsilon}(\cdot, t)$ in the sequel.

The above uniform bound on $\mathcal{P}_{\epsilon}$, with respect to $\epsilon$, is a special feature that distinguishes this discussion from the homogenization theory of time dependent PDEs where the associated operators are typically unbounded elliptic partial differential operators from $L^{p}\left(\Omega ; \mathbb{R}^{k}\right)$ to $L^{p}\left(\Omega ; \mathbb{R}^{k}\right)$.

For applications to periydnamics, we are also interested in the equation of motion which involves second order time derivatives such as

$$
\left\{\begin{array}{l}
\mathbf{u}_{t t}^{\epsilon}(t)=\mathcal{P}_{\epsilon} \mathbf{u}^{\epsilon}(t)+\mathbf{b}_{\epsilon}(t) \\
\mathbf{u}^{\epsilon}(0)=\mathbf{u}_{0}^{\epsilon} \text { and } \mathbf{u}_{t}^{\epsilon}(0)=\mathbf{v}_{0}^{\epsilon}
\end{array}\right.
$$

It can be formulated as an abstract evolution equation in $L^{p}\left(\Omega ; \mathbb{R}^{k}\right) \times L^{p}\left(\Omega ; \mathbb{R}^{k}\right)$ given by

$$
\left\{\begin{aligned}
\mathbf{U}_{t}^{\epsilon}(t) & =\mathfrak{P}_{\epsilon} \mathbf{U}^{\epsilon}+\mathfrak{b}_{\epsilon} \\
\mathbf{U}^{\epsilon}(0) & =\mathbf{U}_{0}^{\epsilon}
\end{aligned}\right.
$$

where

$$
\mathbf{U}^{\epsilon}(t)=\left(\begin{array}{c}
\mathbf{u}^{\epsilon}(t) \\
\partial_{t} \mathbf{u}^{\epsilon}(t)
\end{array}\right), \mathbf{U}_{0}^{\epsilon}=\left(\begin{array}{c}
\mathbf{u}_{0}^{\epsilon} \\
\mathbf{v}_{0}^{\epsilon}
\end{array}\right), \mathfrak{b}^{\epsilon}(t)=\left(\begin{array}{c}
0 \\
\mathbf{b}^{\epsilon}(t)
\end{array}\right) \text {, and } \mathfrak{P}_{\epsilon}=\left(\begin{array}{cc}
0 & I \\
\mathcal{P}^{\epsilon} & 0
\end{array}\right)
$$

Here the notation $I$ denotes the identity map in $L^{p}\left(\Omega ; \mathbb{R}^{k}\right)$ and we also have

$$
\sup _{\epsilon>0}\left\|\mathfrak{P}_{\epsilon}\right\| \leq C,
$$

We easily see that the forms of (2.1) and (2.3) are the same except formal notational change. Thus any result presented for (2.1) can be readily modified for (2.3). For completeness, we present the results for (2.1) and (2.2) in parallel without repeating the technical proofs.

\subsection{Existence of a solution to the abstract equation}

The following theorem presents the existence of a unique solution to the abstract time-dependent Problem (2.1) which can be proved by standard semigroup theory $[17,26,28]$. Our main objective is to state the appropriate bounds on the solution which will be used in the multiscale analysis.

Theorem 2.1. Assume that the sequence $\mathbf{u}_{0}^{\epsilon}$ is uniformly bounded in $L^{p}\left(\Omega ; \mathbb{R}^{k}\right)$ with respect to $\epsilon>0$. Suppose that $T>0$ and that $\mathbf{b}^{\epsilon}(\cdot, t)$ is uniformly bounded in $C\left([0, T] ; L^{p}\left(\Omega ; \mathbb{R}^{k}\right)\right)$ with respect to $\epsilon>0$. Then we have the following result.

(1) Equation (2.1) has a unique classical solution $\mathbf{u}^{\epsilon} \in C^{1}\left([0, T] ; L^{p}\left(\Omega ; \mathbb{R}^{k}\right)\right)$ which is given by

$$
\mathbf{u}^{\epsilon}(t)=\mathrm{e}^{t \mathcal{P}_{\epsilon}} \mathbf{u}_{0}^{\epsilon}+\int_{0}^{t} \mathrm{e}^{(t-s) \mathcal{P}_{\epsilon}} \mathbf{b}_{\epsilon}(s) \mathrm{d} s .
$$

(2) The sequences $\left(\mathbf{u}^{\epsilon}\right)_{\epsilon>0}$ is a uniformly bounded sequence in $C^{1}\left([0, T] ; L^{p}\left(\Omega ; \mathbb{R}^{k}\right)\right)$ with respect to $\epsilon>0$. i.e.

$$
\sup _{\epsilon>0} \sup _{t \in[0, T]}\left\{\left\|\mathbf{u}^{\epsilon}(t)\right\|_{L^{p}}+\left\|\partial_{t} \mathbf{u}^{\epsilon}(t)\right\|_{L^{p}}\right\}<\infty .
$$

The proof of the theorem follows from standard semigroup theory, see for example [28]. The uniformly bounded sequence of linear operators $\mathcal{P}_{\epsilon}$ is a generator of the uniformly continuous semigroup $\left\{\mathrm{e}^{t \mathcal{P}_{\epsilon}}\right\}_{t \geq 0}$, where for each $t$

$$
\mathrm{e}^{t \mathcal{P}_{\epsilon}}=\sum_{n=0}^{\infty} \frac{t^{n}}{n !}\left(\mathcal{P}_{\epsilon}\right)^{n} .
$$


As a consequence the inhomogeneous abstract time-dependent problem (2.1) has a unique classical solution $\mathbf{u}^{\epsilon} \in C^{1}\left([0, T] ; L^{p}\left(\Omega ; \mathbb{R}^{k}\right)\right)$ given by the "variations of parameters" formula (2.4). Moreover, from (2.4) there exists $C>0$ independent of $\epsilon$ such that

$$
\sup _{t \in[0, T]}\left\|\mathbf{u}^{\epsilon}(t)\right\|_{L^{p}\left(\Omega ; \mathbb{R}^{k}\right)} \leq C
$$

that follows from the assumption of the uniform boundedness of $\mathbf{u}_{0}^{\epsilon}, \mathbf{b}_{\epsilon}$ and the fact that $\left\|\mathrm{e}^{t \mathcal{P}_{\epsilon}}\right\| \leq \mathrm{e}^{t M}$, where $M:=\sup _{\epsilon>0}\left\|\mathcal{P}_{\epsilon}\right\|$.

By the equivalence between (2.2) and (2.3), we get as a direct consequence to the above the following result.

Theorem 2.2. Assume that the sequences $\mathbf{u}_{0}^{\epsilon}, \mathbf{v}_{0}^{\epsilon}$ are uniformly bounded in $L^{p}\left(\Omega ; \mathbb{R}^{k}\right)$ with respect to $\epsilon>0$. Suppose that $T>0$ and that $\mathbf{b}^{\epsilon}(\cdot, t)$ is uniformly bounded in $C\left([0, T] ; L^{p}\left(\Omega ; \mathbb{R}^{k}\right)\right)$ with respect to $\epsilon>0$. Then we have the following result.

(1) The sequence $\mathfrak{P}_{\epsilon}$ is a sequence of uniformly bounded linear operators on $L^{p}\left(\Omega ; \mathbb{R}^{k}\right) \times L^{p}\left(\Omega ; \mathbb{R}^{k}\right)$.

(2) Equation (2.2) has a unique classical solution $\mathbf{u}^{\epsilon} \in C^{2}\left([0, T] ; L^{p}\left(\Omega ; \mathbb{R}^{k}\right)\right)$ which is given by

$$
\mathbf{u}^{\epsilon}(t)=\mathrm{C}\left(t \mathcal{P}_{\epsilon}\right) \mathbf{u}_{0}^{\epsilon}+\mathrm{S}\left(t \mathcal{P}_{\epsilon}\right) \mathbf{v}_{0}^{\epsilon}+\int_{0}^{t} \mathbf{S}\left((t-\tau) \mathcal{P}_{\epsilon}\right) \mathbf{b}^{\epsilon}(\tau) \mathrm{d} \tau
$$

(3) The sequences $\left(\mathbf{u}^{\epsilon}\right)_{\epsilon>0}$, is uniformly bounded, with respect to $\epsilon>0$, in $C^{2}\left([0, T] ; L^{p}\left(\Omega ; \mathbb{R}^{k}\right)\right)$. i.e.

$$
\sup _{\epsilon>0} \sup _{t \in[0, T]}\left\{\left\|\mathbf{u}^{\epsilon}(t)\right\|_{L^{p}}+\left\|\partial_{t} \mathbf{u}^{\epsilon}(t)\right\|_{L^{p}}+\left\|\partial_{t}^{2} \mathbf{u}^{\epsilon}(t)\right\|_{L^{p}}\right\}<\infty .
$$

In the above theorem the operators cosh and sinh are defined as

$$
\begin{array}{r}
\mathrm{C}\left(t \mathcal{P}_{\epsilon}\right):=\sum_{n=0}^{\infty} \frac{t^{2 n}}{(2 n) !}\left(\mathcal{P}_{\epsilon}\right)^{n} \\
\mathrm{~S}\left(t \mathcal{P}_{\epsilon}\right):=\sum_{n=0}^{\infty} \frac{t^{2 n+1}}{(2 n+1) !}\left(\mathcal{P}_{\epsilon}\right)^{n}
\end{array}
$$

Using the equation (2.4) on the abstract time-dependent problem (2.3) and taking out the first component of $\mathbf{U}^{\epsilon}$, we obtain an explicit formula for the solution $\mathbf{u}^{\epsilon}$ of (2.2) given in (2.5).

\subsection{Two scale convergence}

In this paper we apply the method of two scale convergence to study the homogenization of equations of type (2.1) and (2.2). We begin with the definition of two scale convergence and state some results. The content of the discussion in this subsection is standard. We are presenting it here to introduce the necessary definitions and to clarify their relations especially for functions that are defined in space and time, as most often such properties are only used for functions of spatial variables in the literature.

\subsubsection{Definitions of two scale convergence}

Let $p$ and $p^{\prime}$ be two real numbers such that $1<p, p^{\prime}<\infty$ and $\frac{1}{p}+\frac{1}{p^{\prime}}=1$. We rely on the definition and properties of vector-valued function spaces such as $L^{1}\left(\Omega ; C_{\text {per }}(Y)\right)$ found in [23]. We want to point out quickly that if $f \in L^{1}\left(\Omega ; C_{\text {per }}(Y)\right)$, then $f$ can be identified with $f(\mathbf{x}, \mathbf{y})$ in $\Omega \times \mathbb{R}^{d}, f$ is of Caratheodory type, $f \in L^{1}(\Omega \times Y)$, and that for all $\epsilon>, f\left(x, \frac{x}{\epsilon}\right)$ is measurable. See ([23], Thm. 4) for other function spaces. 
Definition 2.3 (Two-scale convergence $[3,27]$ ). A sequence $\left(v^{\epsilon}\right)$ of functions in $L^{p}(\Omega)$, is said to two-scale converge to a limit $v \in L^{p}(\Omega \times Y)$ if, as $\epsilon \rightarrow 0$

$$
\int_{\Omega} v^{\epsilon}(\mathbf{x}) \psi\left(\mathbf{x}, \frac{\mathbf{x}}{\epsilon}\right) \mathrm{d} \mathbf{x} \rightarrow \int_{\Omega \times Y} v(\mathbf{x}, \mathbf{y}) \psi(\mathbf{x}, \mathbf{y}) \mathrm{d} \mathbf{x} \mathrm{d} \mathbf{y}
$$

for all $\psi \in L^{p^{\prime}}\left(\Omega ; C_{\text {per }}(Y)\right)$. We often use $v^{\epsilon} \stackrel{2}{\rightarrow} v$ to denote that $\left(v^{\epsilon}\right)$ two-scale converges to $v$.

We note that if $\left(v^{\epsilon}\right)$ is bounded in $L^{p}(\Omega)$, the space $L^{p^{\prime}}\left(\Omega ; C_{\text {per }}(Y)\right)$ can be replaced by $C_{c}^{\infty}\left(\Omega ; C_{\text {per }}^{\infty}(Y)\right)$ in Definition (2.3) (see [23]). A motivation for Definition 2.3 is given by the following compactness result of Nguetseng (see [27] and Allaire [3]).

Proposition 2.4. Let $\left(v^{\epsilon}\right)$ be a bounded sequence in $L^{p}(\Omega)$. Then there exists a subsequence and a function $v \in L^{p}(\Omega \times Y)$ such that the subsequence two-scale converges to $v$.

For time-dependent sequence of functions $v^{\epsilon}(\mathbf{x}, t)$ one slightly modifies the above two-scale convergence to allow for homogenization with a parameter, see $[9,14,15,29,30]$. In this case, the variable $t$ will serve as a parameter, and the modification involves devising a convergence mechanism that does not seek oscillatory behavior in $v^{\epsilon}(\mathbf{x}, t)$ in the $t$ variable. To do that in the definition of the two scale limit we restrict the class of test functions to $\psi \in L^{p^{\prime}}\left(\Omega \times(0, T) ; C_{\text {per }}(Y)\right)$ instead of $\psi \in L^{p^{\prime}}\left(\Omega \times(0, T) ; C_{\text {per }}(Y \times[0,1])\right)$. The following definition makes this precise.

Definition 2.5. A sequence $\left(v^{\epsilon}\right)$ of functions in $L^{p}(\Omega \times(0, T))$, is said to two-scale converge to a limit $v \in$ $L^{p}(\Omega \times(0, T) \times Y)$ if, as $\epsilon \rightarrow 0$

$$
\int_{\Omega \times(0, T)} v^{\epsilon}(\mathbf{x}, t) \psi\left(\mathbf{x}, t, \frac{\mathbf{x}}{\epsilon}\right) \mathrm{d} \mathbf{x} \mathrm{d} t \rightarrow \int_{\Omega \times(0, T) \times Y} v(\mathbf{x}, \mathbf{y}, t) \psi(\mathbf{x}, t, \mathbf{y}) \mathrm{d} \mathbf{x} \mathrm{d} t \mathrm{~d} \mathbf{y}
$$

for all $\psi \in L^{p^{\prime}}\left(\Omega \times(0, T) ; C_{\mathrm{per}}(Y)\right)$.

A two-scale compactness result similar to (and as a consequence of) Proposition 2.4 holds for time dependent bounded sequence of functions as well and is stated in the following proposition.

Proposition 2.6. Let $\left(v^{\epsilon}\right)$ be a bounded sequence in $L^{p}(\Omega \times(0, T))$. Then there exists a subsequence and a function $v \in L^{p}(\Omega \times(0, T) \times Y)$ such that the subsequence two-scale converges to $v$.

Proof. Applying Proposition 2.4 over the domain $\Omega \times(0, T)$ and $Y \times[0,1]$ being the corresponding unit cube, there exists a subsequence (not labeled) and $\tilde{v}(\mathbf{x}, t, \mathbf{y}, \tau) \in L^{p}(\Omega \times(0, T) \times Y \times[0,1])$ such that

$$
\int_{\Omega} v^{\epsilon}(\mathbf{x}, t) \psi\left(\mathbf{x}, t, \frac{\mathbf{x}}{\epsilon}, \frac{t}{\epsilon}\right) \mathrm{d} \mathbf{x} \mathrm{d} t \rightarrow \int_{\Omega \times[0, T] \times Y \times[0,1]} \tilde{v}(\mathbf{x}, t, \mathbf{y}, \tau) \psi(\mathbf{x}, t, \mathbf{y}, \tau) \mathrm{d} \mathbf{x} \mathrm{d} t \mathrm{~d} \mathbf{y} \mathrm{d} \tau
$$

for all $\psi \in L^{p^{\prime}}\left(\Omega \times(0, T) ; C_{\text {per }}(Y \times[0,1])\right)$. Observe that the space inclusion $L^{p^{\prime}}\left(\Omega \times(0, T) ; C_{\text {per }}(Y)\right) \subset$ $L^{p^{\prime}}\left(\Omega \times(0, T) ; C_{\text {per }}(Y \times[0,1])\right)$ holds and that in Definition 2.5 we test the sequence $v_{\epsilon}(\mathbf{x}, t)$ against $\psi \in$ $L^{p^{\prime}}\left(\Omega \times(0, T) ; C_{\mathrm{per}}(Y)\right)$. It then follows from the preceding convergence that as $\epsilon \rightarrow 0$,

$$
\int_{\Omega} v^{\epsilon}(\mathbf{x}, t) \psi\left(\mathbf{x}, t, \frac{\mathbf{x}}{\epsilon}\right) \mathrm{d} \mathbf{x} \mathrm{d} t \rightarrow \int_{\Omega \times[0, T] \times Y \times[0,1]} \tilde{v}(\mathbf{x}, t, \mathbf{y}, \tau) \psi(\mathbf{x}, t, \mathbf{y}) \mathrm{d} \mathbf{x} \mathrm{d} t \mathrm{~d} \mathbf{y} \mathrm{d} \tau
$$

for all $\psi \in L^{p^{\prime}}\left(\Omega \times(0, T) ; C_{\mathrm{per}}(Y)\right)$. Applying Fubini's theorem and iterating the integral, the limit integral can be written as

$$
\int_{\Omega \times[0, T] \times Y \times[0,1]} \tilde{v}(\mathbf{x}, t, \mathbf{y}, \tau) \psi(\mathbf{x}, t, \mathbf{y}) \mathrm{d} \mathbf{x} \mathrm{d} t \mathrm{~d} \mathbf{y} \mathrm{d} \tau=\int_{\Omega \times[0, T] \times Y} v(\mathbf{x}, t, \mathbf{y}) \psi(\mathbf{x}, t, \mathbf{y}) \mathrm{d} \mathbf{x} \mathrm{d} t \mathrm{~d} \mathbf{y}
$$


where $v(\mathbf{x}, t, \mathbf{y})=\int_{0}^{1} \tilde{v}(\mathbf{x}, t, \mathbf{y}, \tau) \mathrm{d} \tau$. To complete the proof we apply Fubini once again to observe that $v \in$ $L^{p}(\Omega \times(0, T) \times Y)$.

For a function $\mathbf{u}(\mathbf{x}, \mathbf{y}) \in L^{p}\left(\Omega \times Y ; \mathbb{R}^{k}\right)$, we may define the average function $\langle\mathbf{u}\rangle(\mathbf{x})$ for almost all $\mathbf{x} \in \Omega$

$$
\langle\mathbf{u}\rangle(\mathbf{x}):=\int_{Y} \mathbf{u}(\mathbf{x}, \mathbf{y}) \mathrm{d} \mathbf{y} .
$$

Note that $\langle\mathbf{u}\rangle \in L^{p}\left(\Omega ; \mathbb{R}^{k}\right)$ and that applying Fubini's theorem and Minkowski's inequality

$$
\|\langle\mathbf{u}\rangle\|_{L^{p}(\Omega)} \leq\|\mathbf{u}\|_{L^{p}(\Omega \times Y)}
$$

Based on the above definitions and together with their natural extensions to vector fields, we record two well known results on two-scale convergence that can be found in [23].

\section{Lemma 2.7.}

1. Let $\left(\mathbf{v}^{\epsilon}\right)$ be a bounded sequence in $L^{p}\left(\Omega \times(0, T) ; \mathbb{R}^{k}\right)$ that two-scale converges to $\mathbf{v} \in L^{p}\left(\Omega \times Y \times(0, T) ; \mathbb{R}^{k}\right)$, then $\mathbf{v}^{\epsilon} \rightarrow\langle\mathbf{v}\rangle(\mathbf{x}, t)$ weakly in $L^{p}\left(\Omega \times(0, T) ; \mathbb{R}^{k}\right)$ as $\epsilon \rightarrow 0$.

2. Suppose $\boldsymbol{\psi}$ is in $L^{p}\left(\Omega ; C_{\mathrm{per}}\left(Y ; \mathbb{R}^{k}\right)\right)$, or $L_{\mathrm{per}}^{p}\left(Y ; C\left(\bar{\Omega} ; \mathbb{R}^{k}\right)\right)$. Then $\boldsymbol{\psi}\left(\mathbf{x}, \frac{\mathbf{x}}{\epsilon}\right)$ two-scale converges to $\boldsymbol{\psi}(\mathbf{x}, \mathbf{y})$ and the sequence of norms also converge:

$$
\lim _{\epsilon \rightarrow 0} \int_{\Omega}\left|\psi\left(\mathbf{x}, \frac{\mathbf{x}}{\epsilon}\right)\right|^{p} \mathrm{~d} \mathbf{x}=\int_{\Omega} \int_{Y}|\boldsymbol{\psi}(\mathbf{x}, \mathbf{y})|^{p} \mathrm{~d} \mathbf{y} \mathrm{d} \mathbf{x} .
$$

Remark 2.8. Given a function $\mathbf{u}(\mathbf{x}, \mathbf{y}, t) \in C\left([0, T] ; L^{p}\left(\Omega ; C_{\text {per }}\left(Y ; \mathbb{R}^{k}\right)\right)\right)$, for each $t \in[0, T], \mathbf{u}(\cdot, \cdot, t) \in$ $L^{p}\left(\Omega ; C_{\mathrm{per}}\left(Y ; \mathbb{R}^{k}\right)\right)$ and as a consequence, as $\epsilon \rightarrow 0$,

$$
\mathbf{u}\left(\mathbf{x}, \frac{\mathbf{x}}{\epsilon}, t\right) \stackrel{2}{\rightarrow} \mathbf{u}(\mathbf{x}, \mathbf{y}, t) \text { in } L^{p}\left(\Omega \times Y ; \mathbb{R}^{k}\right)
$$

with the sequence of norms converging as well; that is for all $t \in[0, T]$,

$$
\lim _{\epsilon \rightarrow 0} \int_{\Omega}\left|\mathbf{u}\left(\mathbf{x}, \frac{\mathbf{x}}{\epsilon}, t\right)\right|^{p} \mathrm{~d} \mathbf{x}=\int_{\Omega} \int_{Y}|\mathbf{u}(\mathbf{x}, \mathbf{y}, t)|^{p} \mathrm{~d} \mathbf{x} \mathrm{d} \mathbf{y} .
$$

Two scale convergence combined with norm convergence is usually referred to as strong two scale convergence, see $[3,14]$.

\subsubsection{Two scale convergence and convolution}

For the problems we will be studying in the next section, the underlying operators are given by finite sums of convolution-type operators. It is thus important to understand the relationship between two scale convergence and the kind of convolution-type operators of interests here. We should mention that the two scale limit of a convolution of an integrable function with a two scale convergent sequence is given in ([14], Prop. 2.13). We will not be using ([14], Prop. 2.13) however, as our operators involve convolution of an integrable function with sequences of functions of both spatial and time variables with the convolution only in the spatial variables. In this direction a result that we will need later is contained in the following theorem. Before stating the theorem, let us give the following elementary result. Given a function $f$ defined on $\Omega$, we denote its extension by zero outside $\Omega$ by $\bar{f}$.

Lemma 2.9. Suppose that $\mathbf{v}(\mathbf{x}, t) \in L^{p}\left(\Omega \times[0, T] ; \mathbb{R}^{k}\right)$ and $g \in L^{1}\left(\mathbb{R}^{k}\right)$. Then the convolution of $g$ and $\mathbf{v}$ in the $\mathbf{x}$-variable defined as

$$
\left(g *_{\mathbf{x}} \mathbf{v}\right)(\mathbf{x}, t)=\int_{\mathbb{R}^{k}} g\left(\mathbf{x}-\mathbf{x}^{\prime}\right) \overline{\mathbf{v}}\left(\mathbf{x}^{\prime}, t\right) \mathrm{d} \mathbf{x}^{\prime}
$$

is in $L^{p}\left(\Omega \times[0, T] ; \mathbb{R}^{k}\right)$ with the estimate

$$
\left\|g *_{\mathbf{X}} \mathbf{v}\right\|_{L^{p}(\Omega \times[0, T])} \leq\|\mathbf{v}\|_{L^{p}(\Omega \times[0, T])}
$$


Proof. Although not difficult, let us show that the function $(\mathbf{x}, t) \mapsto\left(g *_{\mathbf{x}} \mathbf{v}\right)(\mathbf{x}, t)$ is a measurable function in $\mathbb{R}^{k} \times \mathbb{R}$. Clearly the function $\left(\mathbf{x}^{\prime}, \mathbf{x}, t\right) \mapsto g\left(\mathbf{x}-\mathbf{x}^{\prime}\right) \overline{\mathbf{v}}\left(\mathbf{x}^{\prime}, t\right)$ is a measurable function in $\mathbb{R}^{k} \times \mathbb{R}^{k} \times \mathbb{R}$. Iterating the integral, and applying Young's inequality for each $t$, we obtain that

$$
\begin{aligned}
\int_{-\infty}^{\infty} \int_{\mathbb{R}^{k}} \int_{\mathbb{R}^{k}}\left|g\left(\mathbf{x}-\mathbf{x}^{\prime}\right)\right||\overline{\mathbf{v}}|\left(\mathbf{x}^{\prime}, t\right) \mathrm{d} \mathbf{x}^{\prime} \mathrm{d} \mathbf{x} \mathrm{d} t & \leq\|g\|_{L^{1}} \int_{-\infty}^{\infty} \int_{\mathbb{R}^{k}}|\overline{\mathbf{v}}|(\mathbf{x}, t) \mathrm{d} \mathbf{x} \mathrm{d} t \\
& \leq \int_{0}^{T} \int_{\Omega}|\mathbf{v}|(\mathbf{x}, t) \mathrm{d} \mathbf{x} \mathrm{d} t<\infty
\end{aligned}
$$

where the finiteness of the last integral follows from the assumption. And therefore, by Fubini's theorem, $(\mathbf{x}, t) \mapsto\left(g *_{\mathbf{x}} \mathbf{v}\right)(\mathbf{x}, t)$ is a measurable function. The estimate

$$
\left\|g *_{\mathbf{x}} \mathbf{v}\right\|_{L^{p}(\Omega \times[0, T])} \leq\|g\|_{L^{1}}\|\mathbf{v}\|_{L^{p}(\Omega \times[0, T])}
$$

follows from the standard Young's inequality.

Theorem 2.10. Suppose that $\mathbf{u}^{\epsilon}(\mathbf{x}, t)$ is a bounded sequence in $C\left([0, T] ; L^{p}\left(\Omega ; \mathbb{R}^{k}\right)\right)$, and two scale converges to $\mathbf{u}(\mathbf{x}, \mathbf{y}, t)$ in $L^{p}\left(\Omega \times Y \times[0, T] ; \mathbb{R}^{k}\right)$. Then given $g(\xi) \in L^{1}\left(\mathbb{R}^{k}\right)$, there exists a subsequence $\mathbf{u}^{\epsilon_{n}}$ such that the following holds.

(1) For any $\phi \in C[0, T]$, as $n \rightarrow \infty$ the sequence of functions

$$
\mathbf{U}_{\phi}^{\epsilon_{n}}(\mathbf{x}, t)=\int_{-\infty}^{\infty} \int_{\mathbb{R}^{k}} g\left(\mathbf{x}-\mathbf{x}^{\prime}\right) \chi_{[0, T]}\left(t^{\prime}-t\right) \bar{\phi}\left(t^{\prime}\right) \overline{\mathbf{u}}^{\epsilon_{n}}\left(\mathbf{x}^{\prime}, t^{\prime}\right) \mathrm{d} \mathbf{x}^{\prime} \mathrm{d} t^{\prime}
$$

strongly converges in $L^{p}\left(\Omega \times[0, T] ; \mathbb{R}^{k}\right)$ to

$$
\mathbf{U}_{\phi}(\mathbf{x}, t)=\int_{-\infty}^{\infty} \int_{\mathbb{R}^{k}} g\left(\mathbf{x}-\mathbf{x}^{\prime}\right) \chi_{[0, T]}\left(t^{\prime}-t\right) \bar{\phi}\left(t^{\prime}\right)\langle\overline{\mathbf{u}}\rangle\left(\mathbf{x}^{\prime}, t^{\prime}\right) \mathrm{d} \mathbf{x}^{\prime} \mathrm{d} t^{\prime},
$$

where $\chi_{[0, T]}(s)$ is the characteristic function on $[0, T]$.

(2) As $n \rightarrow \infty$, the sequence of convolutions (in $\mathbf{x}$ )

$$
g *_{\mathbf{x}} \overline{\mathbf{u}}^{\epsilon_{n}}(\mathbf{x}, t) \stackrel{2}{\rightarrow} g *_{\mathbf{x}}\langle\overline{\mathbf{u}}\rangle(\mathbf{x}, t) \text { in } L^{p}\left(\Omega \times Y \times[0, T] ; \mathbb{R}^{k}\right)
$$

where the convolution $*_{\mathbf{x}}$ is as defined in Lemma 2.9 .

Proof. 1). We begin noting that since $\mathbf{u}^{\epsilon} \stackrel{2}{\rightarrow} \mathbf{u}$, in $L^{p}\left(\Omega \times[0, T] \times Y ; \mathbb{R}^{k}\right)$, by Lemma 2.7 , we have $\mathbf{u}^{\epsilon} \rightarrow\langle\mathbf{u}\rangle$ weakly in $L^{p}\left(\Omega \times[0, T] ; \mathbb{R}^{k}\right)$, and therefore for any $\phi \in C[0, T], \phi(t) \mathbf{u}^{\epsilon}(\mathbf{x}, t) \rightarrow \phi(t)\langle\mathbf{u}\rangle(\mathbf{x}, t)$ weakly in $L^{p}(\Omega \times[0, T])$ as well. Defining $W(\mathbf{x}, s)=g(\xi) \chi_{[0, T]}(s)$ in $L^{1}\left(\mathbb{R}^{d+1}\right)$, we observe that for any $\phi \in C[0, T],(\mathbf{x}, t) \in \mathbb{R}^{d+1}$,

$$
\mathbf{U}_{\phi}^{\epsilon}(\mathbf{x}, t)=W *\left(\bar{\phi} \overline{\mathbf{u}}^{\epsilon}\right)(\mathbf{x}, t) .
$$

Now using the compactness of the convolution operator (see [8]) and the weak convergence of $\bar{\phi} \overline{\mathbf{u}}^{\epsilon}$, we can extract a subsequence (that may depend on $\phi$ ) so that as $\epsilon \rightarrow 0$,

$$
W *\left(\bar{\phi} \overline{\mathbf{u}}^{\epsilon}\right)(\mathbf{x}, t) \rightarrow W *(\bar{\phi}\langle\overline{\mathbf{u}}\rangle)(\mathbf{x}, t)
$$

strongly in $L^{p}\left(\Omega \times[0, T] ; \mathbb{R}^{k}\right)$. We use now the separability of $C[0, T]$, to remove the dependence of the subsequence on $\phi$. Although the argument is standard, we would like to include it for completeness. Let $\left\{\phi_{k}\right\}_{k=1}^{\infty}$ be a countable dense subset of $C[0, T]$. For each $k$, there exists a subsequence $\epsilon_{n}^{k}$ such that as $n \rightarrow \infty$

$$
W *\left(\bar{\phi}_{k} \overline{\mathbf{u}}^{\epsilon_{n}^{k}}\right)(\mathbf{x}, t) \rightarrow W *\left(\bar{\phi}_{k}\langle\overline{\mathbf{u}}\rangle\right)(\mathbf{x}, t)
$$


strongly in $L^{p}(\Omega \times[0, T])$. Now using a standard diagonalization procedure, we can extract the subsequence $\epsilon_{n}^{n}=\epsilon_{n}$ such that for any $k$, as $n \rightarrow \infty$

$$
W *\left(\bar{\phi}_{k} \overline{\mathbf{u}}^{\epsilon_{n}}\right)(\mathbf{x}, t) \rightarrow W *\left(\bar{\phi}_{k}\langle\overline{\mathbf{u}}\rangle\right)(\mathbf{x}, t)
$$

strongly in $L^{p}(\Omega \times[0, T])$. Now let $\phi \in C[0, T]$, and $\phi_{k^{\prime}} \subset\left\{\phi_{k}\right\}$ such that $\left\|\phi-\phi_{k^{\prime}}\right\|_{L^{\infty}} \rightarrow 0$, as $k^{\prime} \rightarrow \infty$. Then using triangular inequality, for each $n$ and $k^{\prime}$,

$$
\begin{aligned}
& \left\|W *\left(\bar{\phi} \overline{\mathbf{u}}^{\epsilon_{n}}\right)-W *(\bar{\phi}\langle\overline{\mathbf{u}}\rangle)\right\|_{L^{p}(\Omega \times[0, T])} \leq\left\|W *\left(\left(\bar{\phi}-\bar{\phi}_{k^{\prime}}\right) \overline{\mathbf{u}}^{\epsilon_{n}}\right)\right\|_{L^{p}(\Omega \times[0, T])} \\
& +\left\|W *\left(\bar{\phi}_{k^{\prime}} \overline{\mathbf{u}}^{\epsilon_{n}}\right)-W *\left(\bar{\phi}_{k^{\prime}}(\overline{\mathbf{u}}\rangle\right)\right\|_{L^{p}(\Omega \times[0, T])}+\left\|W *\left(\left(\bar{\phi}-\bar{\phi}_{k^{\prime}}\right)\langle\overline{\mathbf{u}}\rangle\right)\right\|_{L^{p}(\Omega \times[0, T])} .
\end{aligned}
$$

Fixing $k^{\prime}$ and letting $n \rightarrow \infty$, we observe that

$$
\begin{aligned}
& \lim _{n \rightarrow \infty}\left\|W *\left(\bar{\phi} \overline{\mathbf{u}}^{\epsilon_{n}}\right)-W *(\bar{\phi}\langle\overline{\mathbf{u}}\rangle)\right\|_{L^{p}(\Omega \times[0, T])} \leq \lim _{n \rightarrow \infty}\left\|W *\left(\left(\bar{\phi}-\bar{\phi}_{k^{\prime}}\right) \overline{\mathbf{u}}^{\epsilon_{n}}\right)\right\|_{L^{p}(\Omega \times[0, T])} \\
& +\lim _{n \rightarrow \infty}\left\|W *\left(\left(\bar{\phi}-\bar{\phi}_{k^{\prime}}\right)\langle\overline{\mathbf{u}}\rangle\right)\right\|_{L^{p}(\Omega \times[0, T]) .}
\end{aligned}
$$

Let us estimate the right hand side. For each $n$ and $k^{\prime}$, using Young's inequality

$$
\begin{aligned}
\left\|W *\left(\left(\bar{\phi}-\bar{\phi}_{k^{\prime}}\right) \overline{\mathbf{u}}^{\epsilon_{n}}\right)\right\|_{L^{p}(\Omega \times[0, T])} & \left.\leq\|W\|_{L^{1}\left(\mathbb{R}^{d+1}\right)} \|\left(\bar{\phi}-\bar{\phi}_{k^{\prime}}\right) \overline{\mathbf{u}}^{\epsilon_{n}}\right) \|_{L^{p}(\Omega \times[0, T])} \\
& \leq\|W\|_{L^{1}\left(\mathbb{R}^{d+1}\right)}\left\|\phi-\phi_{k^{\prime}}\right\|_{L^{\infty}[0, T]}\left\|\overline{\mathbf{u}}^{\epsilon_{n}}\right\|_{L^{p}(\Omega \times[0, T])} \\
& \leq C\left\|\phi-\phi_{k^{\prime}}\right\|_{L^{\infty}[0, T]}
\end{aligned}
$$

where $C$ is independent of $n$ as $\left\|\overline{\mathbf{u}}^{\epsilon_{n}}\right\|_{L^{p}(\Omega \times[0, T])} \leq\left\|\mathbf{u}^{\epsilon}\right\|_{C\left([0, T] ; L^{p}\left(\Omega ; \mathbb{R}^{k}\right)\right)} \leq C^{\prime}<\infty$, by assumption. Therefore the right hand side in (2.6) will approach to 0 as $k^{\prime} \rightarrow 0$. That concludes the proof of part 1$)$.

Let us now prove part 2). We begin by noting that applying Lemma $2.9, g *_{\mathbf{x}} \overline{\mathbf{u}}_{\epsilon}(\mathbf{x}, t)$ is a bounded sequence in $L^{p}\left(\Omega \times[0, T] ; \mathbb{R}^{k}\right)$ and so up to a subsequence two scale converges in $L^{p}\left(\Omega \times Y \times[0, T] ; \mathbb{R}^{k}\right)$. Let us identify the two scale limit. Let $\zeta(t) \in C[0, T]$ and $\psi(\mathbf{x}, \mathbf{y}) \in L^{p^{\prime}}\left(\Omega ; C_{\mathrm{per}}\left(Y ; \mathbb{R}^{k}\right)\right)$. Now using the strong convergence of $\mathbf{U}_{\zeta}^{\epsilon_{n}}(\mathbf{x}, t)$ in $L^{p}(\Omega \times[0, T])$, we see that as $n \rightarrow \infty$

$$
\lim _{n \rightarrow \infty} \int_{0}^{T} \int_{\Omega} \psi\left(\mathbf{x}, \frac{\mathbf{x}}{\epsilon_{n}}\right) \cdot \mathbf{U}_{\zeta}^{\epsilon_{n}}(\mathbf{x}, t) \mathrm{d} \mathbf{x} \mathrm{d} t=\int_{0}^{T} \int_{\Omega} \int_{Y} \psi(\mathbf{x}, \mathbf{y}) \cdot \mathbf{U}_{\zeta}(\mathbf{x}, t) \mathrm{d} \mathbf{y} \mathrm{d} \mathbf{x} \mathrm{d} t .
$$

Let us rewrite each of these integrals separately. We begin iterating the integral in the left hand side, which is allowed using Fubini's theorem as the integral is finite in the product space. It follows then that

$$
\begin{aligned}
& \int_{0}^{T} \int_{\Omega} \psi\left(\mathbf{x}, \frac{\mathbf{x}}{\epsilon_{n}}\right) \cdot \mathbf{U}_{\zeta}^{\epsilon_{n}}(\mathbf{x}, t) \mathrm{d} \mathbf{x} \mathrm{d} t \\
& =\int_{0}^{T} \int_{\Omega} \psi\left(\mathbf{x}, \frac{\mathbf{x}}{\epsilon_{n}}\right) \cdot \int_{-\infty}^{\infty} \int_{\mathbb{R}^{d}} g\left(\mathbf{x}^{\prime}-\mathbf{x}\right) \chi_{[0, T]}\left(t^{\prime}-t\right) \bar{\zeta}\left(t^{\prime}\right) \overline{\mathbf{u}}^{\epsilon_{n}}\left(\mathbf{x}^{\prime}, t^{\prime}\right) \mathrm{d} \mathbf{x}^{\prime} \mathrm{d} t^{\prime} \mathrm{d} \mathbf{x} \mathrm{d} t \\
& =\int_{-\infty}^{\infty} \int_{\Omega} \psi\left(\mathbf{x}, \frac{\mathbf{x}}{\epsilon_{n}}\right) \cdot \int_{\mathbb{R}^{d}} g\left(\mathbf{x}^{\prime}-\mathbf{x}\right)\left(\int_{0}^{T} \chi_{[0, T]}\left(t^{\prime}-t\right) \mathrm{d} t\right) \bar{\zeta}\left(t^{\prime}\right) \overline{\mathbf{u}}^{\epsilon_{n}}\left(\mathbf{x}^{\prime}, t^{\prime}\right) \mathrm{d} \mathbf{x}^{\prime} \mathrm{d} t^{\prime} \mathrm{d} \mathbf{x} \mathrm{d} t \\
& =T \int_{0}^{\infty} \int_{\Omega} \bar{\zeta}\left(t^{\prime}\right) \psi\left(\mathbf{x}, \frac{\mathbf{x}}{\epsilon_{n}}\right) \cdot \int_{\mathbb{R}^{d}} g\left(\mathbf{x}^{\prime}-\mathbf{x}\right) \overline{\mathbf{u}}^{\epsilon_{n}}\left(\mathbf{x}^{\prime}, t^{\prime}\right) \mathrm{d} \mathbf{x}^{\prime} \mathrm{d} \mathbf{x} \mathrm{d} t^{\prime} \\
& =T \int_{0}^{T} \int_{\Omega} \zeta\left(t^{\prime}\right) \psi\left(\mathbf{x}, \frac{\mathbf{x}}{\epsilon_{n}}\right) \cdot\left(g *_{\mathbf{x}} \overline{\mathbf{u}}^{\epsilon_{n}}\right)\left(\mathbf{x}, t^{\prime}\right) \mathrm{d} \mathbf{x} \mathrm{d} t^{\prime}
\end{aligned}
$$

where we used the fact that for all $t^{\prime}, \int_{0}^{T} \chi_{[0, T]}\left(t^{\prime}-t\right) \mathrm{d} t=\int_{0}^{T} \chi_{\left[t^{\prime}-T, t^{\prime}\right]}(t) \mathrm{d} t=T$ Similarly,

$$
\int_{0}^{T} \int_{\Omega} \int_{Y} \psi(\mathbf{x}, \mathbf{y}) \cdot \mathbf{U}_{\zeta}(\mathbf{x}, t) \mathrm{d} \mathbf{y} \mathrm{d} \mathbf{x} \mathrm{d} t=T \int_{0}^{T} \int_{\Omega} \int_{Y} \zeta\left(t^{\prime}\right) \psi(\mathbf{x}, \mathbf{y}) \cdot\left(g *_{\mathbf{x}}\langle\overline{\mathbf{u}}\rangle\right)\left(\mathbf{x}, t^{\prime}\right) \mathrm{d} \mathbf{y} \mathrm{d} \mathbf{x} \mathrm{d} t^{\prime}
$$


Recaping, we have just demonstrated that

$$
\begin{aligned}
\lim _{n \rightarrow \infty} T \int_{0}^{T} \int_{\Omega} \zeta(t) \psi & \left(\mathbf{x}, \frac{\mathbf{x}}{\epsilon_{n}}\right) \cdot\left(g *_{\mathbf{x}} \overline{\mathbf{u}}^{\epsilon_{n}}\right)(\mathbf{x}, t) \mathrm{d} \mathbf{x} \mathrm{d} t \\
= & T \int_{0}^{T} \int_{\Omega} \int_{Y} \zeta(t) \psi(\mathbf{x}, \mathbf{y}) \cdot\left(g *_{\mathbf{x}}\langle\overline{\mathbf{u}}\rangle\right)(\mathbf{x}, t) \mathrm{d} \mathbf{y} \mathrm{d} \mathbf{x} \mathrm{d} t .
\end{aligned}
$$

From the arbitrariness of $\zeta$ and $\psi$ we conclude that the two scale limit of the sequence $g *_{\mathbf{x}} \overline{\mathbf{u}}^{\epsilon_{n}}$ is $g *_{\mathbf{x}}\langle\overline{\mathbf{u}}\rangle$.

Remark 2.11. An important consequence of the above theorem is that if $\mathbb{G}(\mathbf{x})$ is an integrable $\mathbb{R}^{k \times k}$ matrix function and $\mathbf{u}^{\epsilon}$ is a bounded sequence in $C\left([0, T] ; L^{p}\left(\Omega ; \mathbb{R}^{k}\right)\right)$ that two scale converges to $\mathbf{u}(\mathbf{x}, \mathbf{y}, t)$, then as a finite sum of a sequence of convolutions, we can extract a subsequence $\epsilon_{n}$ such that, as $n \rightarrow \infty$, the sequence of functions

$$
\left(\mathbb{G} *_{\mathbf{x}} \overline{\mathbf{u}}^{\epsilon_{n}}\right)(\mathbf{x}, t)=\int_{\Omega} \mathbb{G}\left(\mathbf{x}^{\prime}-\mathbf{x}\right) \mathbf{u}^{\epsilon_{n}}\left(\mathbf{x}^{\prime}, t\right) \mathrm{d} \mathbf{x}^{\prime} \stackrel{2}{\longrightarrow} \mathbb{G} *_{\mathbf{x}}\langle\overline{\mathbf{u}}\rangle(\mathbf{x}, t)=\int_{\Omega} \mathbb{G}\left(\mathbf{x}^{\prime}-\mathbf{x}\right)\langle\mathbf{u}\rangle\left(\mathbf{x}^{\prime}, t\right) \mathrm{d} \mathbf{x}^{\prime}
$$

in $L^{p}\left(\Omega \times[0, T] \times Y ; \mathbb{R}^{k}\right)$.

\subsection{Two scale convergence and time derivatives}

As we have seen at the beginning of the section, we will be dealing with both sequences of functions and their time derivatives. Recall that from our existence result, if $\mathbf{u}_{0}^{\epsilon}(\mathbf{x})$ is bounded in $L^{p}(\Omega)$ and $\mathbf{b}^{\epsilon}(\mathbf{x}, t)$ is bounded in $C\left([0, T] ; L^{p}\left(\Omega ; \mathbb{R}^{k}\right)\right)$, then a unique solution $\mathbf{u}^{\epsilon}(\mathbf{x}, t)$ to the evolution equation (2.1) exists and the sequence of solutions is uniformly bounded in $C^{1}\left([0, T] ; L^{p}\left(\Omega ; \mathbb{R}^{k}\right)\right)$. Similarly, in the event that $\mathbf{U}_{0}^{\epsilon}(\mathbf{x})$ is bounded in $L^{p}\left(\Omega ; \mathbb{R}^{k}\right) \times L^{p}\left(\Omega ; \mathbb{R}^{k}\right)$, then the sequence of solutions $\mathbf{u}^{\epsilon}(\mathbf{x}, t)$ to $(2.2)$ is uniformly bounded in $C^{2}\left([0, T] ; L^{p}\left(\Omega ; \mathbb{R}^{k}\right)\right)$. The following lemma relates the two scale limit of the sequence of functions with the two scale limit of the sequence of time derivatives.

Lemma 2.12 (two-scale convergence and time derivative). Given a bounded sequence $\mathbf{u}^{\epsilon} \in$ $C^{1}\left([0, T] ; L^{p}\left(\Omega ; \mathbb{R}^{k}\right)\right)$, i.e. $\sup _{t \in[0, T]}\left\{\left\|\mathbf{u}^{\epsilon}(t)\right\|_{L^{p}}+\left\|\partial_{t} \mathbf{u}^{\epsilon}(t)\right\|_{L^{p}}\right\}$ is bounded. Suppose also that for almost all $\mathbf{x} \in \Omega, \mathbf{u}^{\epsilon}(\mathbf{x}, 0)=\mathbf{u}_{0}^{\epsilon}(\mathbf{x}), \mathbf{u}_{0}^{\epsilon}(\mathbf{x}) \stackrel{2}{\rightarrow} \mathbf{u}_{0}(\mathbf{x}, \mathbf{y})$ in $L^{p}\left(\Omega \times Y ; \mathbb{R}^{k}\right)$ and $\partial_{t} \mathbf{u}^{\epsilon} \stackrel{2}{\rightarrow} \mathbf{u}^{*}(\mathbf{x}, \mathbf{y}, t)$ in $L^{p}\left(\Omega \times Y \times(0, T) ; \mathbb{R}^{k}\right)$. Define the function

$$
\mathbf{u}(\mathbf{x}, \mathbf{y}, t)=\int_{0}^{t} \mathbf{u}^{*}(\mathbf{x}, \mathbf{y}, \tau) \mathrm{d} \tau+\mathbf{u}_{0}(\mathbf{x}, \mathbf{y})
$$

for almost all $(\mathbf{x}, \mathbf{y}) \in \Omega \times Y$ and all $t \in[0, T]$. Then $\mathbf{u}^{\epsilon} \stackrel{2}{\rightarrow} \mathbf{u}(\mathbf{x}, \mathbf{y}, t)$ in $L^{p}\left(\Omega \times Y \times(0, T) ; \mathbb{R}^{k}\right)$ and $\partial_{t} \mathbf{u}(\mathbf{x}, \mathbf{y}, t)=$ $\mathbf{u}^{*}(\mathbf{x}, \mathbf{y}, t)$. As a consequence $\partial_{t} \mathbf{u}^{\epsilon} \stackrel{2}{\rightarrow} \partial_{t} \mathbf{u}$ in $L^{p}\left(\Omega \times Y \times(0, T) ; \mathbb{R}^{k}\right)$, and $\mathbf{u}^{\epsilon}(\mathbf{x}, 0) \stackrel{2}{\rightarrow} \mathbf{u}(\mathbf{x}, \mathbf{y}, 0)$ in $L^{p}\left(\Omega \times Y ; \mathbb{R}^{k}\right)$.

Proof. Noting that for almost all $t \in[0, T], \mathbf{u}^{*}(\cdot, \cdot, t) \in L^{p}\left(\Omega \times Y ; \mathbb{R}^{k}\right)$, it is not difficult to see that $\mathbf{u}$ is in $C\left([0, T] ; L^{p}\left(\Omega \times Y ; \mathbb{R}^{k}\right)\right), \partial_{t} \mathbf{u}(\mathbf{x}, \mathbf{y}, t)=\mathbf{u}^{*}(\mathbf{x}, \mathbf{y}, t) \in L^{p}\left(\Omega \times Y \times[0, T] ; \mathbb{R}^{k}\right)$, and that for almost all $(\mathbf{x}, \mathbf{y}) \in \Omega \times Y$, $\mathbf{u}(\mathbf{x}, \mathbf{y}, \cdot) \in C[0, T]$. By definition, for all almost $(\mathbf{x}, \mathbf{y}) \in \Omega \times Y$, pointwise evaluation of $\mathbf{u}(\mathbf{x}, \mathbf{y}, \cdot)$ makes sense and $\mathbf{u}(\mathbf{x}, \mathbf{y}, 0)=\mathbf{u}_{0}(\mathbf{x}, \mathbf{y})$. Let us show that $\mathbf{u}^{\epsilon}$ two scale converges to $\mathbf{u}(\mathbf{x}, \mathbf{y}, t)$. Let $\boldsymbol{\psi} \in L^{p^{\prime}}\left(\Omega \times(0, T) ; C_{\mathrm{per}}\left(Y ; \mathbb{R}^{k}\right)\right)$. We will show that as $\epsilon \rightarrow 0$,

$$
\int_{\Omega \times[0, T]} \mathbf{u}^{\epsilon}(\mathbf{x}, t) \cdot \boldsymbol{\psi}\left(\mathbf{x}, \frac{\mathbf{x}}{\epsilon}, t\right) \mathrm{d} \mathbf{x} \mathrm{d} t \rightarrow \int_{\Omega \times(0, T) \times Y} \mathbf{u}(\mathbf{x}, \mathbf{y}, t) \cdot \boldsymbol{\psi}(\mathbf{x}, \mathbf{y}, t) \mathrm{d} \mathbf{x} \mathrm{d} t \mathrm{~d} \mathbf{y} .
$$

Define

$$
\phi(\mathbf{x}, \mathbf{y}, t)=\int_{0}^{t} \boldsymbol{\psi}(\mathbf{x}, \mathbf{y}, \tau) \mathrm{d} \tau-\int_{0}^{T} \psi(\mathbf{x}, \mathbf{y}, \tau) \mathrm{d} \tau .
$$


Then $\phi \in L^{p^{\prime}}\left(\Omega \times(0, T) ; C_{\mathrm{per}}\left(Y ; \mathbb{R}^{k}\right)\right), \partial_{t} \phi=\boldsymbol{\psi}$, and $\boldsymbol{\phi}(x, y, T)=0$. Integrating by parts we obtain that

$$
\begin{aligned}
\int_{\Omega} \int_{0}^{T} \partial_{t} \mathbf{u}^{\epsilon}(\mathbf{x}, t) \phi\left(\mathbf{x}, \frac{\mathbf{x}}{\epsilon}, t\right) \mathrm{d} t \mathrm{~d} \mathbf{x}= & \int_{\Omega} \mathbf{u}^{\epsilon}(x, 0) \int_{0}^{T} \boldsymbol{\psi}\left(\mathbf{x}, \frac{\mathbf{x}}{\epsilon}, \tau\right) \mathrm{d} \tau \mathrm{d} \mathbf{x} \\
& -\int_{\Omega} \int_{0}^{T} \mathbf{u}^{\epsilon}(\mathbf{x}, t) \boldsymbol{\psi}\left(\mathbf{x}, \frac{\mathbf{x}}{\epsilon}, t\right) \mathrm{d} \mathbf{x} \mathrm{d} t .
\end{aligned}
$$

Taking the limit on both sides and using the facts that $\partial_{t} \mathbf{u}^{\epsilon} \stackrel{2}{\rightarrow} \mathbf{u}^{*}, \mathbf{u}_{0}^{\epsilon} \stackrel{2}{\rightarrow} \mathbf{u}_{0}(\mathbf{x}, \mathbf{y})$, and the function $(\mathbf{x}, \mathbf{y}) \mapsto$ $\int_{0}^{T} \psi(\mathbf{x}, \mathbf{y}, \tau) \mathrm{d} \tau$ is an admissible test function, we have

$$
\begin{aligned}
\lim _{\epsilon \rightarrow 0} & \int_{\Omega} \int_{0}^{T} \mathbf{u}^{\epsilon}(\mathbf{x}, t) \cdot \psi\left(\mathbf{x}, \frac{\mathbf{x}}{\epsilon}, t\right) \mathrm{d} \mathbf{x} \mathrm{d} t \\
& =\int_{\Omega \times Y} \int_{0}^{T} \mathbf{u}_{0}(\mathbf{x}, \mathbf{y}) \cdot \boldsymbol{\psi}(\mathbf{x}, \mathbf{y}, t) \mathrm{d} \mathbf{x} \mathrm{d} \mathbf{y} \mathrm{d} t-\int_{\Omega \times Y} \int_{0}^{T} \mathbf{u}^{*}(\mathbf{x}, \mathbf{y}, t) \cdot \phi(\mathbf{x}, \mathbf{y}, t) \mathrm{d} t \mathrm{~d} \mathbf{x} \mathrm{d} \mathbf{y}
\end{aligned}
$$

Integrating by parts the second integral in the right hand side shows that

$$
-\int_{\Omega \times Y} \int_{0}^{T} \mathbf{u}^{*}(\mathbf{x}, \mathbf{y}, t) \cdot \phi(\mathbf{x}, \mathbf{y}, t) \mathrm{d} t \mathrm{~d} \mathbf{x} \mathrm{d} \mathbf{y}=\int_{\Omega \times Y} \int_{0}^{T}\left(\int_{0}^{t} \mathbf{u}^{*}(\mathbf{x}, \mathbf{y}, \tau) \mathrm{d} \tau\right) \cdot \boldsymbol{\psi}(\mathbf{x}, \mathbf{y}, t) \mathrm{d} t \mathrm{~d} \mathbf{x} \mathrm{d} \mathbf{y} .
$$

Combining the above and using the definition of $\mathbf{u}$ we have

$$
\begin{aligned}
\lim _{\epsilon \rightarrow 0} \int_{\Omega} \int_{0}^{T} & \mathbf{u}^{\epsilon}(\mathbf{x}, t) \cdot \boldsymbol{\psi}\left(\mathbf{x}, \frac{\mathbf{x}}{\epsilon}, t\right) \mathrm{d} \mathbf{x} \mathrm{d} t \\
& =\int_{\Omega \times Y \times(0, T)}\left(\mathbf{u}_{0}(\mathbf{x}, \mathbf{y})+\int_{0}^{t} \mathbf{u}^{*}(\mathbf{x}, \mathbf{y}, \tau) \mathrm{d} \tau\right) \cdot \boldsymbol{\psi}(\mathbf{x}, \mathbf{y}, t) \mathrm{d} \mathbf{x} \mathrm{d} \mathbf{y} \mathrm{d} t \\
& =\int_{\Omega \times Y \times(0, T)} \mathbf{u}(\mathbf{x}, \mathbf{y}, t) \cdot \boldsymbol{\psi}(\mathbf{x}, \mathbf{y}, t) \mathrm{d} \mathbf{x} \mathrm{d} \mathbf{y} \mathrm{d} t
\end{aligned}
$$

proving that $\mathbf{u}^{\epsilon} \stackrel{2}{\rightarrow} \mathbf{u}$ in $L^{p}\left(\Omega \times[0, T] \times Y ; \mathbb{R}^{k}\right)$.

Remark 2.13. Given a sequence $\mathbf{u}^{\epsilon}(t)$ that is bounded in $C^{1}\left([0, T] ; L^{p}\left(\Omega ; \mathbb{R}^{k}\right)\right)$ and an $L^{p}$ - bounded sequence of initial values $\mathbf{u}^{\epsilon}(0)$, the lemma provides a means of constructing a two scale limit $\mathbf{u}(\mathbf{x}, \mathbf{y}, t) \in C\left([0, T] ; L^{p}(\Omega \times\right.$ $\left.Y ; \mathbb{R}^{k}\right)$ ) for a subsequence $\mathbf{u}^{\epsilon_{k}}$ with the additional property that the sequence of initial values $\mathbf{u}^{\epsilon_{k}}(0) \stackrel{2}{\rightarrow} \mathbf{u}(\mathbf{x}, \mathbf{y}, 0)$, in $L^{p}\left(\Omega \times Y ; \mathbb{R}^{k}\right)$ and the sequence of time derivatives $\partial_{t} \mathbf{u}^{\epsilon_{k}} \stackrel{2}{\rightarrow} \partial_{t} \mathbf{u}$ in $L^{p}\left(\Omega \times[0, T] \times Y ; \mathbb{R}^{d}\right)$. Indeed, starting with the $L^{p}$-bounded sequences, $\partial_{t} \mathbf{u}^{\epsilon}$ and $\mathbf{u}^{\epsilon}(0)$, we can extract a subsequence $\epsilon_{k}$ such that, as $k \rightarrow \infty, \partial \mathbf{u}^{\epsilon_{k}}$ and $\mathbf{u}^{\epsilon_{k}}$ two scale converges to $\mathbf{u}^{*}(\mathbf{x}, \mathbf{y}, t)$ and $\mathbf{u}_{0}(\mathbf{x}, \mathbf{y})$ in $L^{p}$ respectively. We now use Lemma 2.12 to construct $\mathbf{u}(\mathbf{x}, \mathbf{y}, t)$.

The following is a consequence of Lemma 2.12 as well as its counterpart for (2.2). We notice that the results follow exactly the same argument as in the proof of Lemma 2.12. For an alternate proof of the following lemma see $[2]$.

Lemma 2.14. Let $\mathbf{u}^{\epsilon} \in C^{2}\left([0, T] ; L^{p}\left(\Omega ; \mathbb{R}^{k}\right)\right)$ be a bounded sequence; i.e.

$$
\sup _{\epsilon>0} \sup _{t \in[0, T]}\left\{\left\|\mathbf{u}^{\epsilon}(t)\right\|_{L^{p}}+\left\|\partial_{t} \mathbf{u}^{\epsilon}(t)\right\|_{L^{p}}+\left\|\partial_{t}^{2} \mathbf{u}^{\epsilon}(t)\right\|_{L^{p}}\right\}<\infty .
$$

Suppose that for almost all $\mathbf{x} \in \Omega, \mathbf{u}^{\epsilon}(\mathbf{x}, 0)=\mathbf{u}_{0}^{\epsilon}(\mathbf{x}), \partial_{t} \mathbf{u}^{\epsilon}(\mathbf{x}, 0)=\mathbf{v}_{0}^{\epsilon}(\mathbf{x}), \mathbf{u}_{0}^{\epsilon} \stackrel{2}{\rightarrow} \mathbf{u}_{0}(\mathbf{x}, \mathbf{y}), \mathbf{v}_{0}^{\epsilon} \stackrel{2}{\rightarrow} \mathbf{v}_{0}(\mathbf{x}, \mathbf{y})$ in $L^{p}\left(\Omega \times Y ; \mathbb{R}^{k}\right)$ and $\partial_{t}^{2} \mathbf{u}^{\epsilon} \stackrel{2}{\longrightarrow} \mathbf{u}^{* *}(\mathbf{x}, \mathbf{y}, t) \in L^{p}\left(\Omega \times Y \times(0, T) ; \mathbb{R}^{k}\right)$. Define the function

$$
\mathbf{u}(\mathbf{x}, \mathbf{y}, t)=\int_{0}^{t} \int_{0}^{\tau} \mathbf{u}^{* *}(\mathbf{x}, \mathbf{y}, l) \mathrm{d} l \mathrm{~d} \tau+t \mathbf{v}_{0}(\mathbf{x}, \mathbf{y})+\mathbf{u}_{0}(\mathbf{x}, \mathbf{y})
$$


for almost all $(\mathbf{x}, \mathbf{y}) \in \Omega \times Y$ and all $t \in[0, T]$. Then $\mathbf{u}^{\epsilon} \stackrel{2}{\rightarrow} \mathbf{u}(\mathbf{x}, \mathbf{y}, t), \partial_{t} \mathbf{u}^{\epsilon} \stackrel{2}{\rightarrow} \partial_{t} \mathbf{u}(\mathbf{x}, \mathbf{y}, t)$, and $\partial_{t}^{2} \mathbf{u}(\mathbf{x}, \mathbf{y}, t)=$ $\mathbf{u}^{* *}(\mathbf{x}, \mathbf{y}, t)$. As a consequence $\partial_{t}^{2} \mathbf{u}^{\epsilon} \stackrel{2}{\longrightarrow} \partial_{t}^{2} \mathbf{u}(\mathbf{x}, \mathbf{y}, t) \in L^{p}\left(\Omega \times Y \times(0, T) ; \mathbb{R}^{k}\right), \partial_{t} \mathbf{u}^{\epsilon}(\mathbf{x}, 0) \stackrel{2}{\longrightarrow} \partial_{t} \mathbf{u}(\mathbf{x}, \mathbf{y}, 0)$ and $\mathbf{u}^{\epsilon}(\mathbf{x}, 0) \stackrel{2}{\rightarrow} \mathbf{u}(\mathbf{x}, \mathbf{y}, 0)$ in $L^{p}\left(\Omega \times Y ; \mathbb{R}^{k}\right)$.

\subsection{Standard asymptotic analysis}

The asymptotic analysis program we are going to implement in the next sections to model equations of type (2.1) and (2.2) involves two steps: identify the dynamics associated with the two scale limit of solutions, and prove a strong approximation to the solution using a properly scaled solution to the two scale limit equation. Let us discuss the two steps separately.

\subsubsection{Two scale limit equation}

We have seen that the sequence of solutions $\mathbf{u}^{\epsilon}$ to (2.1) associated with uniformly bounded linear operators $\mathcal{P}_{\epsilon}$ is bounded in $C^{1}\left([0, T] ; L^{p}\left(\Omega ; \mathbb{R}^{k}\right)\right)$ provided that the initial data $\mathbf{u}_{0}^{\epsilon}$ is bounded in $L^{p}\left(\Omega ; \mathbb{R}^{k}\right)$ and the body force $\mathbf{b}_{\epsilon}(\mathbf{x}, t)$ is bounded in $C\left([0, T] ; L^{p}\left(\Omega ; \mathbb{R}^{k}\right)\right)$. The sequence of solutions $\mathbf{u}^{\epsilon}$, thus, has a subsequence that two scale converges to say, $\mathbf{u}(\mathbf{x}, \mathbf{y}, t)$ in $L^{p}\left(\Omega \times[0, T] \times Y ; \mathbb{R}^{k}\right)$. One then inquires if there is a dynamics associated with $\mathbf{u}$. In fact, for the model problems that we will be dealing in this paper, we will prove the existence of a "limiting" bounded linear operator $\mathcal{P}_{0}$ in $L^{p}\left(\Omega \times Y ; \mathbb{R}^{k}\right)$ such that $\mathbf{u}$ actually satisfies the system

$$
\partial_{t} \mathbf{u}(\mathbf{x}, \mathbf{y}, t)=\mathcal{P}_{0} \mathbf{u}(\mathbf{x}, \mathbf{y}, t)+\mathbf{b}(\mathbf{x}, \mathbf{y}, t)
$$

supplemented with initial conditions

$$
\mathbf{u}(\mathbf{x}, \mathbf{y}, 0)=\mathbf{u}_{0}(\mathbf{x}, \mathbf{y}),
$$

where $\mathbf{u}_{0}(\mathbf{x}, \mathbf{y})$, and $\mathbf{b}(\mathbf{x}, \mathbf{y}, t)$ are two scale limits of $\mathbf{u}_{0}^{\epsilon}$ and $\mathbf{b}_{\epsilon}$ respectively. Applying the general existence of a solution, if $\mathbf{b}(\mathbf{x}, \mathbf{y}, t) \in C\left([0, T] ; L^{p}(\Omega \times Y)\right)$, then $\mathbf{u}$ is uniquely defined. This in turn implies not only a subsequence but also the whole sequence $\mathbf{u}^{\epsilon}$ two scale converges to $\mathbf{u}$.

\subsubsection{Strong approximation by two scale functions}

The two scale convergence of the solutions $\mathbf{u}^{\epsilon}$ of the evolution equation implies the weak convergence $\mathbf{u}_{\epsilon} \rightarrow\langle\mathbf{u}\rangle$, weakly in $L^{p}(\Omega \times[0, T])$, and that for any subdomain $V \subset \Omega$, and any interval $\left(t_{0}, t_{1}\right)$,

$$
\lim _{\epsilon \rightarrow 0} \int_{V \times\left(t_{0}, t_{1}\right)} \mathbf{u}^{\epsilon}(\mathbf{x}, t) \mathrm{d} \mathbf{x} \mathrm{d} t=\lim _{\epsilon \rightarrow 0} \int_{V \times\left(t_{0}, t_{1}\right)}\langle\mathbf{u}\rangle(\mathbf{x}, t) \mathrm{d} \mathbf{x} \mathrm{d} t .
$$

Defining $\mathbf{u}^{H}(\mathbf{x})=\langle\mathbf{u}\rangle(\mathbf{x})$, we may write $\mathbf{u}(\mathbf{x}, \mathbf{y}, t)=\mathbf{u}^{H}(\mathbf{x}, t)+\mathbf{w}(\mathbf{x}, \mathbf{y}, t)$. Then $\mathbf{w} \in L^{p}\left(\Omega \times Y \times[0, T] ; \mathbb{R}^{d}\right)$ and $\langle\mathbf{w}\rangle(\mathbf{x}, t)=0$ for all $(\mathbf{x}, t) \in \Omega \times[0, T]$. Plugging this decomposition in the two scale limit equation (2.7), one may obtain dynamics of "the homogenized limit" $\mathbf{u}^{H}(\mathbf{x}, t)$ and "the corrector" $\mathbf{w}(\mathbf{x}, \mathbf{y}, t)$. The goal in this second step is to prove that for each $t \in[0, T]$

$$
\left\|\mathbf{u}^{\epsilon}(\cdot, t)-\mathbf{u}\left(\cdot, \frac{\cdot}{\epsilon}, t\right)\right\|_{L^{p}} \rightarrow 0
$$

as $\epsilon \rightarrow 0$, in the event that $\mathbf{u}\left(\mathbf{x}, \frac{\mathbf{x}}{\epsilon}, t\right)$ is a measurable function. Note here that $\mathbf{u}\left(\mathbf{x}, \frac{\mathbf{x}}{\epsilon}, t\right)=\mathbf{u}^{H}(\mathbf{x}, t)+\mathbf{w}\left(\mathbf{x}, \frac{\mathbf{x}}{\epsilon}, t\right)$ and we see that $\mathbf{w}\left(\mathbf{x}, \frac{\mathbf{x}}{\epsilon}, t\right)$ plays the role of corrector. We also observe that for almost all $t \in[0, T], \mathbf{u}(\cdot, \cdot, t)$ is merely in $L^{p}\left(\Omega \times Y ; \mathbb{R}^{k}\right)$ and thus the function $\mathbf{u}\left(\mathbf{x}, \frac{\mathbf{x}}{\epsilon}, t\right)$ may not be measurable unless $\mathbf{u}(\mathbf{x}, \mathbf{y}, t)$ belongs to a smoother function space. As a solution to a system of model equations, we know in fact that $\mathbf{u}$ does belong to a smoother function space when additional regularity on the data $\mathbf{b}, \mathbf{u}_{0}$ and $\mathbf{v}_{0}$ is imposed. 


\section{HomogenizATION OF NONLOCAL CONVECTION-DIFFUSION}

We consider a special variant of the homogeneous linear nonlocal convection-diffusion model studied in [13]

$$
\partial_{t} u^{\epsilon}(\mathbf{x}, t)=\mathcal{L}_{\epsilon} u^{\epsilon}(\mathbf{x}, t) \text { for } \mathbf{x} \in \Omega, t>0,
$$

for a real-valued function $u^{\epsilon}=u^{\epsilon}(\mathbf{x}, t)$ satisfying the initial condition given by

$$
u^{\epsilon}(\mathbf{x}, 0)=u_{0}^{\epsilon}(\mathbf{x}), \quad \forall \mathbf{x} \in \Omega .
$$

The nonlocal convection-diffusion operator $\mathcal{L}_{\epsilon}$ is defined by

$$
\mathcal{L}_{\epsilon}(v)(\mathbf{x})=\int_{\Omega}\left(\gamma_{\epsilon}\left(\mathbf{x}^{\prime}, \mathbf{x}\right) v\left(\mathbf{x}^{\prime}\right)-\gamma_{\epsilon}\left(\mathbf{x}, \mathbf{x}^{\prime}\right) v(\mathbf{x})\right) \mathrm{d} \mathbf{x}^{\prime}
$$

for a non-negative interaction kernel $\gamma_{\epsilon}$.

When $\Omega$ is a bounded domain, the equation (3.1) imposes natural modifications to the nonlocal interactions near the boundary so that no additional boundary or volume conditions are required [11]. We refer to [13] for more detailed discussions. Here for mathematical convenience we specialize the kernel $\gamma_{\epsilon}$ to get a nonlocal operator $\mathcal{L}_{\epsilon}$ of the form

$$
\mathcal{L}_{\epsilon}(u)(\mathbf{x})=\int_{\Omega} \rho\left(\mathbf{x}^{\prime}-\mathbf{x}\right)\left(\Theta_{\epsilon}\left(\mathbf{x}^{\prime}\right) u\left(\mathbf{x}^{\prime}\right)-\Theta_{\epsilon}(\mathbf{x}) u(\mathbf{x})\right) \mathrm{d} \mathbf{x}^{\prime}
$$

where in this case $\gamma_{\epsilon}\left(\mathbf{x}^{\prime}, \mathbf{x}\right)=\rho\left(\mathbf{x}^{\prime}-\mathbf{x}\right) \Theta_{\epsilon}\left(\mathbf{x}^{\prime}\right)$ and $\rho=\rho(\boldsymbol{\xi})$ is a locally integrable, even and nonnegative density and $\Theta_{\epsilon}(\mathbf{x})=\Theta\left(\mathbf{x}, \frac{\mathbf{x}}{\epsilon}\right)$ measures heterogeneity. The function $\Theta=\Theta(\mathbf{x}, \mathbf{y})$ is assumed to belong to $L^{\infty}\left(\Omega ; C_{\mathrm{per}}(Y)\right)$ and is uniformly bounded from above and below by some positive constants. Note that we have made $\rho$ to be independent of $\epsilon$ which simplifies the analysis, and at the same time, also allows us to highlight the difference from other kernels used in the peridynamic operators considered in the next section.

\subsection{Two scale limit equation}

It is not difficult to show that the nonlocal convection-diffusion operator $\mathcal{L}_{\epsilon}$ given in (3.3) is a bounded linear operator on $L^{p}(\Omega)$ [13]. Moreover, the operator norm is uniformly bounded from above in $\epsilon$. One may easily obtain the well-posedness of the equation from Theorem 2.1 (see also [13]). Let us introduce an operator $\mathcal{L}_{0}$ given by

$$
\left(\mathcal{L}_{0} \mathbf{u}\right)(\mathbf{x}, \mathbf{y})=\int_{\Omega} \rho\left(\mathbf{x}^{\prime}-\mathbf{x}\right)\left(\langle\Theta u\rangle\left(\mathbf{x}^{\prime}\right)-\Theta(\mathbf{x}, \mathbf{y}) u(\mathbf{x}, \mathbf{y})\right) \mathrm{d} \mathbf{x}^{\prime} .
$$

It is not difficult to prove that $\mathcal{L}_{0}: L^{p}(\Omega \times Y) \rightarrow L^{p}(\Omega \times Y)$ is a bounded linear operator.

Theorem 3.1. Suppose that $u_{\epsilon}(\mathbf{x}, t)$ is the sequence of solutions to the nonlocal convection-diffusion initial value problem (3.1)-(3.2), with the sequence of initial values $u_{0}^{\epsilon} \stackrel{2}{\rightarrow} u_{0}(\mathbf{x}, \mathbf{y})$ in $L^{p}(\Omega \times Y)$. Suppose also that $\mathcal{L}_{0}$ is the operator defined by (3.4). Then there exists a unique $u=u(\mathbf{x}, \mathbf{y}, t) \in C^{1}\left([0, T] ; L^{p}(\Omega \times Y)\right)$ such that $u_{\epsilon} \stackrel{2}{\rightarrow} u$ in $L^{p}$ and $u$ solves the equation

$$
\partial_{t} u(\mathbf{x}, \mathbf{y}, t)=\left(\mathcal{L}_{0} u\right)(\mathbf{x}, \mathbf{y}, t),(\mathbf{x}, \mathbf{y}) \in \Omega \times Y, t>0
$$

with the initial condition

$$
u(\mathbf{x}, \mathbf{y}, 0)=u_{0}(\mathbf{x}, \mathbf{y}) .
$$


Proof. Step 1. By the existence result Theorem 2.1 and the remark following Lemma 2.12, there exists and subsequence $\epsilon_{n}$ and $u \in C\left([0, T] ; L^{p}(\Omega \times Y)\right)$ with the property that $\partial_{t} u \in L^{p}(\Omega \times Y \times[0, T])$ and $u(\mathbf{x}, \mathbf{y}, 0)=$ $u_{0}(\mathbf{x}, \mathbf{y})$ and as $n \rightarrow \infty$

$$
u^{\epsilon_{n}} \stackrel{2}{\rightarrow} u \quad \text { and } \quad \partial_{t} u^{\epsilon_{n}} \stackrel{2}{\rightarrow} \partial_{t} u
$$

in $L^{p}(\Omega \times Y \times[0, T])$. We further claim that there exists a sub subsequence $\epsilon_{n_{j}}$ such that as $j \rightarrow \infty$

$$
\mathcal{L}_{\epsilon_{n_{j}}} u^{\epsilon_{n_{j}}} \stackrel{2}{\rightarrow} \mathcal{L}_{0} u
$$

in $L^{p}(\Omega \times Y \times[0, T])$. Indeed, we first write $\mathcal{L}_{\epsilon}$ as a sum of two operators as

$$
\mathcal{L}_{\epsilon_{n}} u^{\epsilon_{n}}(\mathbf{x}, t)=\rho *_{\mathbf{x}}\left(\bar{\Theta}_{\epsilon_{n}} \bar{u}^{\epsilon_{n}}\right)(\mathbf{x}, t)-\Theta_{\epsilon_{n}}(\mathbf{x}) u^{\epsilon_{n}}(\mathbf{x}, t) \int_{\Omega} \rho\left(\mathbf{x}-\mathbf{x}^{\prime}\right) \mathrm{d} \mathbf{x}^{\prime}
$$

We then note the fact that $\Theta(\mathbf{x}, \mathbf{y}) \in L^{\infty}\left(\Omega ; C_{\text {per }}(Y)\right), \Theta_{\epsilon} \stackrel{2}{\rightarrow} \Theta(\mathbf{x}, \mathbf{y})$ in $L^{p}(\Omega \times Y)$, as a result, when $n \rightarrow \infty$ the product two scale converges

$$
\Theta_{\epsilon_{n}} u^{\epsilon_{n}} \stackrel{2}{\longrightarrow} \Theta(\mathbf{x}, \mathbf{y}) u(\mathbf{x}, \mathbf{y}, t)
$$

in $L^{p}(\Omega \times[0, T] \times Y)$. Now applying part 2) of Theorem 2.10 we can extract a subsequence $n_{j}$ such that as $j \rightarrow \infty$

$$
\rho *_{\mathbf{x}}\left(\bar{\Theta}_{\epsilon_{n_{j}}} \bar{u}^{\epsilon_{n_{j}}}\right)(\mathbf{x}, t) \stackrel{2}{\rightarrow} \rho *_{\mathbf{x}}\langle\bar{\Theta} \bar{u}\rangle(\mathbf{x}, t)
$$

in $L^{p}(\Omega \times Y \times[0, T])$. For this subsequence, the two scale limit of the second part of $\mathcal{L}_{\epsilon_{n}} \mathbf{u}^{\epsilon_{n}}$ can be found as $j \rightarrow \infty$

$$
\Theta_{\epsilon_{n_{j}}}(\mathbf{x}) u^{\epsilon_{n_{j}}}(\mathbf{x}, t) \int_{\Omega} \rho\left(\mathbf{x}-\mathbf{x}^{\prime}\right) \mathrm{d} \mathbf{x}^{\prime} \stackrel{2}{\longrightarrow} \Theta(\mathbf{x}, \mathbf{y}) u(\mathbf{x}, \mathbf{y}, t) \int_{\Omega} \rho\left(\mathbf{x}-\mathbf{x}^{\prime}\right) \mathrm{d} \mathbf{x}^{\prime}
$$

in $L^{p}(\Omega \times Y \times[0, T])$. Combining the parts, and letting $j \rightarrow \infty$ on both sides of (3.1) we compute the two scale limits and obtain equation (3.5), $u$ satisfying the initial value $u(\mathbf{x}, \mathbf{y}, 0)=u_{0}(\mathbf{x}, \mathbf{y}) \in L^{p}(\Omega \times Y)$. Now using the fact that $\mathcal{L}_{0}$ is a linear bounded operator on $L^{p}(\Omega \times Y)$, we conclude, from the existence theorem, that $u$ is unique. Moreover, using the same argument as above, any two scale converging subsequence of $u^{\epsilon}$ will satisfy equation (3.5) together with the initial value $u_{0}(\mathbf{x}, \mathbf{y})$; and by uniqueness of the solution the two scale limit must be $u$. And therefore, the entire sequence $u^{\epsilon}$ two scale converges to the solution to equation (3.5).

Remark 3.2. We make two remarks. The first one is that for each $t$, the solution $u(\mathbf{x}, \mathbf{y}, t)$ to (3.5) will belong to the same space as the initial condition $u_{0}$ provided that the operator $\mathcal{L}_{0}$ is a bounded operator on that function space. An important space that we use in the sequel is $L^{p}\left(\Omega ; C_{\text {per }}(Y)\right)$ for any $1<p<\infty$. From the simple structure of the limiting operator $\mathcal{L}_{0}$, it is not difficult to show that $\mathcal{L}_{0}$ is indeed a linear bounded operator on $L^{p}\left(\Omega ; C_{\mathrm{per}}(Y)\right)$. The implication of having $u \in C^{1}\left([0, T] ; L^{p}\left(\Omega ; C_{\mathrm{per}}(Y)\right)\right)$, is that for each $t$, the

map $\mathbf{x} \mapsto u\left(\mathbf{x}, \frac{\mathbf{x}}{\epsilon}, t\right)$ is a measurable function. We will use this property to obtain a strong approximation result.

Second, given the fact that $\Theta(\mathbf{x}, \mathbf{y})$ is periodic in the $\mathbf{y}$-variable, we note that the periodic extension of $u(\mathbf{x}, \mathbf{y}, t)$ (we will keep the notation the same) in the $\mathbf{y}$-variable will also solve (3.5) in $\Omega \times \mathbb{R}^{d}$, provided the initial data $u_{0}(\mathbf{x}, \mathbf{y})$ is also periodic in the $\mathbf{y}$-variable. This is the case if $u_{0} \in L^{p}\left(\Omega ; C_{\text {per }}(Y)\right)$, for example.

\subsection{Homogenized nonlocal convection-diffusion model}

From the two scale convergence of $\mathbf{u}^{\epsilon}$ to $\mathbf{u}$, we obtain that as $\epsilon \rightarrow 0$,

$$
u^{\epsilon} \rightarrow u^{H}(\mathbf{x}, t)=\int_{Y} u(\mathbf{x}, \mathbf{y}, t) \mathrm{d} \mathbf{y}
$$


weakly in $L^{p}(\Omega \times[0, T])$. In this subsection we obtain a dynamics for $u^{H}$. Let us begin by writing the operator $\mathcal{L}_{0}$ given in $(3.4)$ as

$$
\left(\mathcal{L}_{0} u\right)(\mathbf{x}, \mathbf{y})=\mathcal{L}_{\text {ave }}^{1} u^{H}(\mathbf{x})+\left(\mathcal{L}_{\text {osc }}^{1} u^{H}\right)(\mathbf{x}, \mathbf{y})+\left(\mathcal{M}\left(u-u^{H}\right)\right)(\mathbf{x}, \mathbf{y})
$$

with the operators on the right hand side defined by

$$
\begin{aligned}
\left(\mathcal{L}_{\text {ave }}^{1} u^{H}\right)(\mathbf{x}) & =\int_{\Omega} \rho_{0}\left(\mathbf{x}^{\prime}-\mathbf{x}\right)\langle\Theta\rangle\left(\mathbf{x}^{\prime}\right)\left(u^{H}\left(\mathbf{x}^{\prime}\right)-u^{H}(\mathbf{x})\right) \mathrm{d} \mathbf{x}^{\prime} \\
\left(\mathcal{L}_{\text {osc }}^{1} u^{H}\right)(\mathbf{x}, \mathbf{y}) & =\left(\int_{\Omega} \rho_{0}\left(\mathbf{x}^{\prime}-\mathbf{x}\right)\left(\langle\Theta\rangle\left(\mathbf{x}^{\prime}\right)-\Theta(\mathbf{x}, \mathbf{y})\right) \mathrm{d} \mathbf{x}^{\prime}\right) u^{H}(\mathbf{x}) \\
(\mathcal{M} w)(\mathbf{x}, \mathbf{y}) & =\int_{\Omega} \rho_{0}\left(\mathbf{x}^{\prime}-\mathbf{x}\right)\left(\langle\Theta w\rangle\left(\mathbf{x}^{\prime}\right)-\Theta(\mathbf{x}, \mathbf{y}) w(\mathbf{x}, \mathbf{y})\right) \mathrm{d} \mathbf{x}^{\prime}
\end{aligned}
$$

We note that

$$
\left\langle\mathcal{L}_{\mathrm{osc}}^{1} u^{H}\right\rangle(\mathbf{x})=\left(\int_{\Omega} \rho\left(\mathbf{x}^{\prime}-\mathbf{x}\right)\left(\langle\Theta\rangle\left(\mathbf{x}^{\prime}\right)-\langle\Theta\rangle(\mathbf{x})\right) \mathrm{d} \mathbf{x}^{\prime}\right) u^{H}(\mathbf{x})
$$

and

$$
\langle\mathcal{M} w\rangle(\mathbf{x})=\int_{\Omega} \rho_{0}\left(\mathbf{x}^{\prime}-\mathbf{x}\right)\left(\langle\Theta w\rangle\left(\mathbf{x}^{\prime}\right)-\langle\Theta w\rangle(\mathbf{x})\right) \mathrm{d} \mathbf{x}^{\prime}
$$

Now write $u(\mathbf{x}, \mathbf{y}, t)=u^{H}(\mathbf{x}, t)+w(\mathbf{x}, \mathbf{y}, t)$. Then $w \in L^{p}(\Omega \times Y \times[0, T])$ and $\langle w\rangle(\mathbf{x}, t)=0$ for all $(\mathbf{x}, t) \in$ $\Omega \times[0, T]$. We thus get

$$
u_{t}^{H}(\mathbf{x}, t)=\mathcal{L}_{\text {ave }}^{1} u^{H}(\mathbf{x}, t)+\left\langle\mathcal{L}_{\text {osc }}^{1} u^{H}\right\rangle(\mathbf{x}, t)+\langle\mathcal{M} w\rangle(\mathbf{x}, t) .
$$

and

$$
w_{t}(\mathbf{x}, \mathbf{y}, t)=\left(\mathcal{M}^{(0)} w\right)(\mathbf{x}, \mathbf{y}, t)+\mathcal{L}_{\text {osc }}^{1(0)} u^{H}(\mathbf{x}, \mathbf{y}, t),
$$

where $\left(\mathcal{M}^{(0)} w\right)(\mathbf{x}, \mathbf{y}, t):=(\mathcal{M} w)(\mathbf{x}, \mathbf{y}, t)-\langle\mathcal{M} w\rangle(\mathbf{x}, t)$ and

$$
\begin{aligned}
\mathcal{L}_{\mathrm{osc}}^{1(0)} u^{H}(\mathbf{x}, \mathbf{y}, t) & =\mathcal{L}_{\mathrm{osc}}^{1} u^{H}(\mathbf{x}, \mathbf{y}, t)-\left\langle\mathcal{L}_{\mathrm{osc}}^{1} u^{H}\right\rangle(\mathbf{x}, t) \\
& =(\langle\Theta\rangle(\mathbf{x})-\Theta(\mathbf{x}, \mathbf{y})) \int_{\Omega} \rho\left(\mathbf{x}^{\prime}-\mathbf{x}\right) \mathrm{d} \mathbf{x}^{\prime} u^{H}(\mathbf{x}, t)
\end{aligned}
$$

The initial data are given by

$$
u^{H}(\mathbf{x}, 0)=\left\langle u_{0}\right\rangle(\mathbf{x}), \quad w(\mathbf{x}, \mathbf{y}, 0)=w_{0}(\mathbf{x}, \mathbf{y})=u_{0}(\mathbf{x}, \mathbf{y})-\left\langle u_{0}\right\rangle(\mathbf{x}) .
$$

One may solve the initial value problem for $w$ first as a function of $u^{H}$ and may utilize the formula

$$
w(\mathbf{x}, \mathbf{y}, t)=\mathrm{e}^{t \mathcal{M}^{(0)}} w_{0}(\mathbf{x}, \mathbf{y})+\int_{0}^{t} \mathrm{e}^{(t-s) \mathcal{M}^{(0)}} \mathcal{L}_{\text {osc }}^{1(0)} u^{H}(\mathbf{x}, \mathbf{y}, s) \mathrm{d} s,
$$

to get

$$
\begin{aligned}
u_{t}^{H}(\mathbf{x}, t)=\mathcal{L}_{\text {ave }}^{1} u^{H}(\mathbf{x}, t)+\left\langle\mathcal{L}_{\text {osc }}^{1} u^{H}\right\rangle(\mathbf{x}, t) \\
\quad+\int_{0}^{t}\left\langle\mathcal{M} \mathrm{e}^{(t-s) \mathcal{M}^{(0)}} \mathcal{L}_{\text {osc }}^{1(0)} u^{H}\right\rangle(\mathbf{x}, s) \mathrm{d} s+\left\langle\mathcal{M} \mathrm{e}^{t \mathcal{M}^{(0)}} w_{0}\right\rangle(\mathbf{x}, t) .
\end{aligned}
$$

The above equation actually demonstrates a nonlocal version of a well known fact from evolution PDEs that whenever there is an oscillatory coefficient (in our case $\langle\Theta\rangle(\mathbf{x})-\Theta(\mathbf{x}, \mathbf{y})$ is not identically zero), the homogenized dynamics exhibits a history dependence, see [34]. 


\subsection{Strong approximation to nonlocal convection-diffusion}

In this subsection, we prove that for each $t \in[0, T]$, the re-scaled function $u\left(\mathbf{x}, \frac{\mathbf{x}}{\epsilon}, t\right)$ of the solution to the two scale limit problem, can be used to approximate the original $u^{\epsilon}(\mathbf{x}, t)$. The main theorem we will be proving is the following.

Theorem 3.3. Let $\left(u^{\epsilon}\right)$ be the sequence of solutions of (3.1)-(3.2) with initial data $u_{0}^{\epsilon}=\mathbf{u}_{0}\left(\mathbf{x}, \frac{\mathbf{x}}{\epsilon}\right)$ where $u_{0} \in$ $L^{p}\left(\Omega ; C_{\mathrm{per}}(Y)\right)$. Then the solution $u$ of the two scale limit equation $(3.5)-(3.6)$ is in $C^{1}\left((0, T) ; L^{p}\left(\Omega ; C_{\mathrm{per}}(Y)\right)\right)$. Moreover, for each $t \in[0, T], u\left(\mathbf{x}, \frac{\mathbf{x}}{\epsilon}, t\right)$ give the strong approximation to $u^{\epsilon}$ as

$$
\lim _{\epsilon \rightarrow 0}\left\|u^{\epsilon}(\cdot, t)-u\left(\cdot, \frac{\dot{\epsilon}}{\epsilon}, t\right)\right\|_{L^{p}(\Omega)}=0 .
$$

Proof. The fact that $u \in C^{1}\left((0, T) ; L^{p}\left(\Omega ; C_{\mathrm{per}}(Y)\right)\right)$, when $u_{0} \in L^{p}\left(\Omega ; C_{\mathrm{per}}(Y)\right)$ follows from standard Semigroup theory as $\mathcal{L}_{0}$ is a bounded linear operator on $L^{p}\left(\Omega ; C_{\text {per }}(Y)\right)$ as discussed in Remark 3.2. What remains is to show the $L^{p}$-strong approximation.

Step 1. Let us identify an evolution equation for the error $\mathrm{e}^{\epsilon}(\mathbf{x}, t)=u^{\epsilon}(\mathbf{x}, t)-u\left(\mathbf{x}, \frac{\mathbf{x}}{\epsilon}, t\right)$. We begin by first writing the dynamics for $u\left(\mathbf{x}, \frac{\mathbf{x}}{\epsilon}, t\right)$, by plugging $\mathbf{y}=\frac{\mathbf{x}}{\epsilon}$ in equation (3.5) to obtain that

$$
\partial_{t} u\left(\mathbf{x}, \frac{\mathbf{x}}{\epsilon}, t\right)=\left(\mathcal{L}_{0} \mathbf{u}\right)\left(\mathbf{x}, \frac{\mathbf{x}}{\epsilon}, t\right),
$$

together with the initial condition $u\left(\mathbf{x}, \frac{\mathbf{x}}{\epsilon}, 0\right)=u_{0}\left(\mathbf{x}, \frac{\mathbf{x}}{\epsilon}\right)$. Subtract this equation from the original abstract equation (3.1)-(3.2). Denoting the difference $e^{\epsilon}(\mathbf{x}, t):=u^{\epsilon}(\mathbf{x}, t)-u\left(\mathbf{x}, \frac{\mathbf{x}}{\epsilon}, t\right)$, it follows that

$$
\left\{\begin{array}{l}
\partial_{t} e^{\epsilon}=\mathcal{L}_{\epsilon} e^{\epsilon}(\mathbf{x}, t)+D^{\epsilon}(\mathbf{x}, t) \quad \mathbf{x} \in \Omega, t>0 \\
e_{\epsilon}(\mathbf{x}, 0)=0, \mathbf{x} \in \Omega
\end{array}\right.
$$

where $D^{\epsilon}(\mathbf{x}, t)=\mathcal{L}^{\epsilon}\left(u\left(\mathbf{x}, \frac{\mathbf{x}}{\epsilon}, t\right)\right)-\left(\mathcal{L}_{0} u\right)\left(\mathbf{x}, \frac{\mathbf{x}}{\epsilon}, t\right)$.

Step 2. To make the evolution equation (3.8) wellposed in $L^{p}(\Omega)$, and to be able to estimate the solution $\mathrm{e}^{\epsilon}$, let us show that for all $\epsilon>0, D^{\epsilon}(\mathbf{x}, t) \in C\left([0, T], L^{p}(\Omega)\right)$ and

$$
\lim _{\epsilon \rightarrow 0}\left\|D^{\epsilon}(\cdot, t)\right\|_{L^{p}(\Omega)}=0, \text { and } \sup _{\epsilon>0} \sup _{t \in[0, T]}\left\|D^{\epsilon}(\cdot, t)\right\|_{L^{p}(\Omega)}<\infty .
$$

To that end, let us first note that simplifying $D^{\epsilon}$ we have that

$$
D^{\epsilon}(\mathbf{x}, t)=\int_{\Omega} \rho\left(\mathbf{x}^{\prime}-\mathbf{x}\right)\left(\Theta\left(\mathbf{x}^{\prime}, \frac{\mathbf{x}^{\prime}}{\epsilon}\right) u\left(\mathbf{x}^{\prime}, \frac{\mathbf{x}^{\prime}}{\epsilon}, t\right)-\langle\Theta u\rangle\left(\mathbf{x}^{\prime}, t\right)\right) \mathrm{d} \mathbf{x}^{\prime} .
$$

It is then clear that there exits a positive constant $\mathrm{C}$ such that for each $t$ and $\epsilon, D^{\epsilon}(\mathbf{x}, t) \in L^{p}(\Omega)$ (after some simplification)

$$
\left\|D^{\epsilon}(\cdot, t)\right\|_{L^{p}(\Omega)} \leq C\|u(\cdot, \cdot, t)\|_{L^{p}(\Omega \times Y)} .
$$

We then have that for each $\epsilon>0$,

$$
\sup _{t \in[0, T]}\left\|D^{\epsilon}(\cdot, t)\right\|_{L^{p}(\Omega)} \leq C \sup _{t \in[0, T]}\|u(\cdot, \cdot, t)\|_{L^{p}(\Omega \times Y)} \leq C\|u\|_{L^{p}\left(\Omega ; C_{\mathrm{per}}(Y)\right)} .
$$

The last inequality shows that the estimate is uniform in $\epsilon$. Similarly, it is not difficult to show that for any $t_{1}, t_{2} \in[0, T]$ and any $\epsilon$

$$
\left\|D^{\epsilon}\left(\cdot, t_{1}\right)-D^{\epsilon}\left(\cdot, t_{2}\right)\right\|_{L^{p}(\Omega)} \leq C\left\|u\left(\cdot, \cdot, t_{1}\right)-u\left(\cdot, \cdot, t_{2}\right)\right\|_{L^{p}(\Omega \times Y)}
$$


from which we use the fact that $u \in C\left([0, T] ; L^{p}\left(\Omega ; C_{\mathrm{per}}(Y)\right)\right)$ to conclude that $D^{\epsilon}(\mathbf{x}, t) \in C\left([0, T] ; L^{p}(\Omega)\right)$. Lastly, the strong $L^{p}$-convergence of $D^{\epsilon}(\cdot, t)$ to 0 follows from the fact that the convolution operator is a compact operator. Indeed, for each $t$, as $\epsilon \rightarrow 0$

$$
\Theta\left(\mathbf{x}, \frac{\mathbf{x}}{\epsilon}\right) u\left(\mathbf{x}, \frac{\mathbf{x}}{\epsilon}, t\right) \rightarrow\langle\Theta u\rangle(\mathbf{x}, t)
$$

weakly in $L^{p}(\Omega)$. Therefore from (3.9) and the above observation we conclude that as $\epsilon \rightarrow 0$

$$
D^{\epsilon}(\mathbf{x}, t)=\rho *_{\mathbf{x}}\left(\bar{\Theta}_{\epsilon} \bar{u}(\cdot, \bar{\epsilon}, t)-\overline{\langle\Theta u\rangle}(\cdot, t)\right)(\mathbf{x}) \rightarrow 0,
$$

strongly in $L^{p}(\Omega)$.

Step 3. We are now ready to estimate $\left\|\mathrm{e}^{\epsilon}(\cdot, t)\right\|_{L^{p}(\Omega)}$. The unique solution $e^{\epsilon} \in C^{1}\left([0, T] ; L^{p}(\Omega)\right)$ of $(3.8)$ is given by the formula,

$$
e^{\epsilon}(\mathbf{x}, t)=\int_{0}^{t} \sum_{n=0}^{\infty} \frac{(t-\tau)^{n}}{n !}\left(\mathcal{L}_{\epsilon}\right)^{n} D^{\epsilon}(\mathbf{x}, \tau) \mathrm{d} \tau .
$$

For fixed $t \in[0, T]$, we now estimate the $L^{p}$ norm of $\mathbf{e}^{\epsilon}$ to obtain that

$$
\begin{aligned}
\left\|e^{\epsilon}(\cdot, t)\right\|_{L^{p}(\Omega)} & \leq \int_{0}^{t} \sum_{n=0}^{\infty} \frac{(t-\tau)^{n}}{(n) !}\left\|\mathcal{L}_{\epsilon}\right\|^{n}\left\|D^{\epsilon}(\cdot, \tau)\right\|_{L^{p}(\Omega)} \\
& \leq \int_{0}^{t} \sum_{n=0}^{\infty} \frac{(t-\tau)^{n}}{(n) !} M^{n}\left\|D^{\epsilon}(\cdot, \tau)\right\|_{L^{p}(\Omega)} \mathrm{d} \tau \\
& \leq \int_{0}^{t} \exp (M(t-\tau))\left\|D^{\epsilon}(\cdot, \tau)\right\|_{L^{p}(\Omega)} \mathrm{d} \tau
\end{aligned}
$$

where we have used the uniform estimate assumption on the operators $\mathcal{L}_{\epsilon}$; that is there exists $M>0$, so that

$$
\sup _{\epsilon>0}\left\|\mathcal{L}_{\epsilon}\right\| \leq M .
$$

Using the facts we proved in Step 2 on $D^{\epsilon}$ and the uniform convergence theorem, we see that $\left\|e^{\epsilon}(\cdot, t)\right\|_{L^{p}\left(\Omega ; \mathbb{R}^{d}\right)} \rightarrow$ 0 , as $\epsilon \rightarrow 0$. That completes the theorem.

\section{Multiscale approximation and homogenization of Linear PERIDynamics}

We consider the state-based linear peridynamic model given in [32]. For convenience, we use the same notation as that given in [24] which is consistent with the original definition given in [32]. A bond based peridynamic model for fiber reinforced materials is considered in [2]. The elastic displacement inside the heterogeneous body $\Omega$ with density $\mathfrak{p}_{\epsilon}$ is denoted by $\mathbf{u}^{\epsilon}(x, t)$ and satisfies the peridynamic equation of motion given by

$$
\mathfrak{p}_{\epsilon}(\mathbf{x}) \partial_{t t} \mathbf{u}^{\epsilon}(\mathbf{x}, t)=\mathcal{L}_{\epsilon} \mathbf{u}^{\epsilon}(\mathbf{x}, t)+\mathbf{b}^{\epsilon}(\mathbf{x}, t), \text { for } \mathbf{x} \in \Omega, t>0,
$$

The initial conditions are given by

$$
\mathbf{u}^{\epsilon}(\mathbf{x}, 0)=\mathbf{u}_{0}^{\epsilon}(\mathbf{x}), \quad \partial_{t} \mathbf{u}^{\epsilon}(\mathbf{x}, 0)=\mathbf{v}_{0}^{\epsilon}(\mathbf{x}), \quad \forall \mathbf{x} \in \Omega .
$$

Here $\mathbf{b}^{\epsilon}(x, t)$ is a body force and by the notation of [24], the operator $\mathcal{L}_{\epsilon}$ is defined as,

$$
\begin{aligned}
\mathcal{L}_{\epsilon}(\mathbf{u})(\mathbf{x})= & \int_{\Omega}\left(\alpha_{\epsilon}(\mathbf{x})+\alpha_{\epsilon}\left(\mathbf{x}^{\prime}\right)\right) \frac{\rho_{\epsilon}\left(\mathbf{x}, \mathbf{x}^{\prime}-\mathbf{x}\right)}{\left|\mathbf{x}^{\prime}-\mathbf{x}\right|^{2}}\left(\mathbf{x}^{\prime}-\mathbf{x}\right)\left(\mathbf{x}^{\prime}-\mathbf{x}\right) \cdot\left(\mathbf{u}\left(\mathbf{x}^{\prime}\right)-\mathbf{u}(\mathbf{x})\right) \mathrm{d} \mathbf{x}^{\prime} \\
& +\int_{\Omega} \tau_{\epsilon}\left(\mathbf{x}^{\prime}\right) \rho_{\epsilon}\left(\mathbf{x}, \mathbf{x}-\mathbf{x}^{\prime}\right)\left(\mathbf{x}^{\prime}-\mathbf{x}\right)\left(\int_{\Omega} \rho_{\epsilon}\left(\mathbf{x}^{\prime}, \mathbf{x}^{\prime}-\mathbf{z}\right)\left(\mathbf{z}-\mathbf{x}^{\prime}\right) \cdot\left(\mathbf{u}(\mathbf{z})-\mathbf{u}\left(\mathbf{x}^{\prime}\right)\right) \mathrm{d} \mathbf{z}\right) \mathrm{d} \mathbf{x}^{\prime} \\
& +\int_{\Omega} \tau_{\epsilon}(\mathbf{x}) \rho_{\epsilon}\left(\mathbf{x}, \mathbf{x}-\mathbf{x}^{\prime}\right)\left(\mathbf{x}^{\prime}-\mathbf{x}\right)\left(\int_{\Omega} \rho_{\epsilon}(\mathbf{x}, \mathbf{x}-\mathbf{z})(\mathbf{z}-\mathbf{x}) \cdot(\mathbf{u}(\mathbf{z})-\mathbf{u}(\mathbf{x})) \mathrm{d} \mathbf{z}\right) \mathrm{d} \mathbf{x}^{\prime}
\end{aligned}
$$


where

$$
\rho_{\epsilon}(\mathbf{x}, \boldsymbol{\xi})=\rho_{0}(\boldsymbol{\xi})+\beta\left(\frac{\mathbf{x}}{\epsilon}, \frac{\mathbf{x}+\boldsymbol{\xi}}{\epsilon}\right) \rho_{2}^{\epsilon}(\boldsymbol{\xi}),
$$

$\beta(\cdot, \cdot)$ is a continuous periodic symmetric function, $\beta\left(\mathbf{y}, \mathbf{y}^{\prime}\right)=\beta\left(\mathbf{y}^{\prime}, \mathbf{y}\right)$, that is bounded from above and below by positive constants, $\rho_{0}(\boldsymbol{\xi})$ is an integrable, positive and even function supported on $B(0, \gamma)$, the ball centered at 0 of radius $\gamma, \rho_{2}^{\epsilon}(\boldsymbol{\xi})=\frac{1}{\epsilon^{d}} \rho_{2}\left(\frac{\boldsymbol{\xi}}{\epsilon}\right)$, and $\rho_{2}(\xi)$ is an integrable, positive and even function with support contained in $B(0, \delta)$. In addition, $\alpha_{\epsilon}(\mathbf{x})$ and $k_{\epsilon}(\mathbf{x})$ are the shear and bulk moduli of the material occupying the position $\frac{\mathbf{x}}{\epsilon}$,

$$
\mathfrak{p}_{\epsilon}(\mathbf{x})=\mathfrak{p}\left(\frac{\mathbf{x}}{\epsilon}\right), \alpha_{\epsilon}(\mathbf{x})=\alpha\left(\frac{\mathbf{x}}{\epsilon}\right), k_{\epsilon}(\mathbf{x})=k\left(\frac{\mathbf{x}}{\epsilon}\right)
$$

and

$$
m_{\epsilon}(\mathbf{x})=\int_{\Omega} \rho_{\epsilon}\left(\mathbf{x}, \mathbf{x}^{\prime}-\mathbf{x}\right)\left|\mathbf{x}^{\prime}-\mathbf{x}\right|^{2} \mathrm{~d} \mathbf{x}^{\prime}
$$

where $\mathfrak{p}, \alpha, k \in C_{\text {per }}(Y)$ are uniformly bounded from above and below by some positive constants, and the scalar function

$$
\tau_{\epsilon}(\mathbf{x})=\frac{d^{2} k_{\epsilon}(\mathbf{x})}{m_{\epsilon}(\mathbf{x})^{2}}-\frac{\alpha_{\epsilon}(\mathbf{x})}{m_{\epsilon}(\mathbf{x})}
$$

Observe that for any $\mathbf{x}^{\prime}, \mathbf{x} \in \Omega$

$$
\rho_{\epsilon}\left(\mathbf{x}, \mathbf{x}^{\prime}-\mathbf{x}\right)=\rho_{\epsilon}\left(\mathbf{x}, \mathbf{x}-\mathbf{x}^{\prime}\right)=\rho_{\epsilon}\left(\mathbf{x}^{\prime}, \mathbf{x}^{\prime}-\mathbf{x}\right) .
$$

\section{Remark 4.1.}

a) We would like to make the observation that $\mathcal{L}_{\epsilon} \mathbf{u}^{\epsilon}$ represents the linearized total internal force applied at material point $\mathbf{x}$ and in our formulation is made up of the force due to long-range interactions (represented by the kernel $\left.\rho_{0}\right)$ and the short range one represented by $\rho_{2}$. The function $\beta(\mathbf{x}, \mathbf{y})$ tracks the properties of two interacting material points. A simple change of variables shows that with the scaling given above, the short range forces act within $\epsilon \delta$ neighborhood of $\mathbf{x}$. The $\epsilon^{-d}$ scaling is a technical artifact that is chosen for $\rho_{2}^{\epsilon}(\boldsymbol{\xi})$ so that the asymptotic dynamics is of peridynamic form. Other choices can be made and this is the subject of future study. For the derivation of the linearized peridynamics equation of motion (4.1) and related discussion on peridynamics see references $[12,22,24,32,33]$, see also $[10,16]$ for recent reviews on the mathematics of peridynamics.

b) We also note that since our interest is at the nonlocal level, we will take $\gamma$ and $\delta$ as given parameters with fixed values. Certainly, estimates for the operator and solutions will depend on $\gamma$ and $\delta$ and we will neither track nor quantify that dependance. In passing, we mention that for fixed $\epsilon$ and when $\rho_{2}=0$, with proper scaling of $\rho_{0}$ with respect to the horizon $\gamma$, it is shown in $[24,32]$ that for small $\gamma$

$$
\mathcal{L}_{\epsilon} \mathbf{u} \approx \operatorname{div}\left(\mu_{\epsilon}(\mathbf{x}) \nabla \mathbf{u}\right)+\nabla\left[\left(\mu_{\epsilon}(\mathbf{x})+\lambda_{\epsilon}(\mathbf{x})\right) \operatorname{div} \mathbf{u}\right]
$$

for a function $\mathbf{u}$ that is either smooth or have properly defined local and nonlocal derivatives. The asymptotic relationship associate with different scalings of the heterogeneity length scale $\epsilon$ and the nonlocal horizon $\gamma$ is a subject of future study.

Remark 4.2. From the conditions imposed above, the weighted volume

$$
m_{0}(\mathbf{x})=\int_{\Omega} \rho_{0}\left(\mathbf{x}^{\prime}-\mathbf{x}\right)\left|\mathbf{x}^{\prime}-\mathbf{x}\right|^{2} \mathrm{~d} \mathbf{x}^{\prime}
$$


is a positive continuous function on $\bar{\Omega}$, (and so $\min _{\mathbf{x} \in \Omega} m_{0}(\mathbf{x})>0$ ). Moreover, $\lim _{\epsilon \rightarrow 0}\left\|m_{\epsilon}-m_{0}\right\|_{L^{\infty}}=0$ and there exists $\epsilon_{0}>0$ and a constant $C>0$, such that

$$
\sup _{\epsilon \in\left(0, \epsilon_{0}\right)} \sup _{\mathbf{x} \in \Omega} \tau_{\epsilon}(\mathbf{x}) \leq C .
$$

\subsection{Properties of PD operators}

To apply the preliminary results we obtained in Section 2 to the peridynamic model, we need to demonstrate that the peridynamic operator satisfies the linearity and boundedness assumptions. Comparing operators, we note that $\mathcal{P}_{\epsilon}$ becomes $\mathfrak{p}_{\epsilon}^{-1} \mathcal{L}_{\epsilon}$. Because of the nondegeneracy assumption on the density $\mathfrak{p}_{\epsilon}$, essentially all properties of $\mathcal{L}_{\epsilon}$ can easily be shown to hold for $\mathcal{P}_{\epsilon}$.

We present the following result on the uniform boundedness of the nonlocal peridynamic operators which extends the uniform boundedness of $\mathcal{L}_{\epsilon}: L^{2}\left(\Omega ; \mathbb{R}^{d}\right) \rightarrow L^{2}\left(\Omega ; \mathbb{R}^{d}\right)$ demonstrated in [24] to any $L^{p}$ space.

Theorem 4.3. The peridynamic operator $\mathcal{L}_{\epsilon}: L^{p}\left(\Omega ; \mathbb{R}^{d}\right) \rightarrow L^{p}\left(\Omega ; \mathbb{R}^{d}\right)$ is a bounded linear operator. Moreover, the operator norm is uniformly bounded from above in $\epsilon$.

Proof. We write $\mathcal{L}^{\epsilon}=L_{b}^{\epsilon}+L_{s 1}^{\epsilon}+L_{s 2}^{\epsilon}$. We will show that each of these maps are bounded linear operators on $L^{p}\left(\Omega ; \mathbb{R}^{d}\right)$. In the above decomposition,

$$
\begin{gathered}
L_{b}^{\epsilon} \mathbf{u}(\mathbf{x})=\int_{\Omega}\left(\alpha_{\epsilon}(\mathbf{x})+\alpha_{\epsilon}\left(\mathbf{x}^{\prime}\right)\right) \frac{\rho_{\epsilon}\left(\mathbf{x}, \mathbf{x}^{\prime}-\mathbf{x}\right)}{\left|\mathbf{x}^{\prime}-\mathbf{x}\right|^{2}}\left(\mathbf{x}^{\prime}-\mathbf{x}\right)\left(\mathbf{x}^{\prime}-\mathbf{x}\right) \cdot\left(\mathbf{u}\left(\mathbf{x}^{\prime}\right)-\mathbf{u}(\mathbf{x})\right) \mathrm{d} \mathbf{x}^{\prime} \\
L_{s 1}^{\epsilon} \mathbf{u}(\mathbf{x})=\int_{\Omega} \tau_{\epsilon}\left(\mathbf{x}^{\prime}\right) \rho_{\epsilon}\left(\mathbf{x}, \mathbf{x}-\mathbf{x}^{\prime}\right)\left(\mathbf{x}^{\prime}-\mathbf{x}\right)\left(\int_{\Omega} \rho_{\epsilon}\left(\mathbf{x}^{\prime}, \mathbf{x}^{\prime}-\mathbf{z}\right)\left(\mathbf{z}-\mathbf{x}^{\prime}\right) \cdot\left(\mathbf{u}(\mathbf{z})-\mathbf{u}\left(\mathbf{x}^{\prime}\right)\right) \mathrm{d} \mathbf{z}\right) \mathrm{d} \mathbf{x}^{\prime}
\end{gathered}
$$

and

$$
L_{s 2}^{\epsilon} \mathbf{u}(\mathbf{x})=\int_{\Omega} \tau_{\epsilon}(\mathbf{x}) \rho_{\epsilon}\left(\mathbf{x}, \mathbf{x}-\mathbf{x}^{\prime}\right)\left(\mathbf{x}^{\prime}-\mathbf{x}\right)\left(\int_{\Omega} \rho_{\epsilon}(\mathbf{x}, \mathbf{x}-\mathbf{z})(\mathbf{z}-\mathbf{x}) \cdot(\mathbf{u}(\mathbf{z})-\mathbf{u}(\mathbf{x})) \mathrm{d} \mathbf{z}\right) \mathrm{d} \mathbf{x}^{\prime}
$$

It is obvious that they are all linear operators. Let us show that they map $L^{p}(\Omega)$ into $L^{p}(\Omega)$. Let us begin estimating. First

$$
\left|L_{b}^{\epsilon} \mathbf{u}(\mathbf{x})\right| \leq g^{\epsilon} *|\overline{\mathbf{u}}|(\mathbf{x})+f^{\epsilon}(\mathbf{x})|\mathbf{u}|(\mathbf{x})
$$

where $g^{\epsilon}(\boldsymbol{\xi})=2 A \rho_{0}(\boldsymbol{\xi})+B \rho_{2}^{\epsilon}(\boldsymbol{\xi})$, and $f^{\epsilon}(\mathbf{x})=2 A m_{\epsilon}(\mathbf{x})$ with $A=\max _{x \in \Omega} \alpha(\mathbf{x}), B=\max _{\mathbf{y}, \mathbf{y}^{\prime} \in Y} \beta\left(\mathbf{y}, \mathbf{y}^{\prime}\right)$. Then we can estimate the $L^{p}$ norm of the first term in the right hand side as

$$
\left\|g^{\epsilon} *|\overline{\mathbf{u}}|\right\|_{L^{p}(\Omega)} \leq\left\|g^{\epsilon}\right\|_{L^{1}\left(\mathbb{R}^{d}\right)}\|\mathbf{u}\|_{L^{p}(\Omega)} .
$$

It is not difficult to show that

$$
\sup _{\epsilon>0}\left\|g^{\epsilon}\right\|_{L^{1}\left(\mathbb{R}^{d}\right)}<\infty \text { and } \sup _{\epsilon>0} \sup _{\mathbf{x} \in \Omega} f^{\epsilon}(\mathbf{x})<\infty .
$$

Combining the above estimates, there exists a positive constant $C$ independent of $\epsilon$ such that

$$
\left\|L_{b}^{\epsilon} \mathbf{u}\right\|_{L^{p}(\Omega)} \leq C\|\mathbf{u}\|_{L^{p}(\Omega)}
$$

To show the boundedness of other operators, define the sequence of operators $\mathbf{u} \mapsto \Gamma_{\epsilon}(\mathbf{u})$ given by

$$
\Gamma^{\epsilon}(\mathbf{u})\left(\mathbf{x}^{\prime}\right)=\int_{\Omega} \rho_{\epsilon}\left(\mathbf{x}^{\prime}, \mathbf{x}^{\prime}-\mathbf{z}\right)\left(\mathbf{z}-\mathbf{x}^{\prime}\right) \cdot\left(\mathbf{u}(\mathbf{z})-\mathbf{u}\left(\mathbf{x}^{\prime}\right)\right) \mathrm{d} \mathbf{z} .
$$


Following the above procedure, we have the estimate

$$
\left\|\Gamma^{\epsilon}(\mathbf{u})\right\|_{L^{p}(\Omega)} \leq C\|\mathbf{u}\|_{L^{p}(\Omega)},
$$

where $\mathrm{C}$ is independent of $\epsilon$. Now we may write

$$
L_{s 1}^{\epsilon} \mathbf{u}(\mathbf{x})=\int_{\Omega} \tau_{\epsilon}\left(\mathbf{x}^{\prime}\right) \rho_{\epsilon}\left(\mathbf{x}, \mathbf{x}-\mathbf{x}^{\prime}\right)\left(\mathbf{x}^{\prime}-\mathbf{x}\right) \Gamma^{\epsilon}(\mathbf{u})\left(\mathbf{x}^{\prime}\right) \mathrm{d} \mathbf{x}^{\prime},
$$

and

$$
L_{s 2}^{\epsilon} \mathbf{u}(\mathbf{x})=\left(\tau_{\epsilon}(\mathbf{x}) \int_{\Omega} \rho_{\epsilon}\left(\mathbf{x}, \mathbf{x}-\mathbf{x}^{\prime}\right)\left(\mathbf{x}^{\prime}-\mathbf{x}\right) \mathrm{d} \mathbf{x}^{\prime}\right) \Gamma^{\epsilon}(\mathbf{u})(\mathbf{x})
$$

Thus we can estimate

$$
\left\|L_{s i}^{\epsilon} \mathbf{u}\right\|_{L^{p}} \leq C\left\|\Gamma^{\epsilon}(\mathbf{u})\right\|_{L^{p}}, \text { for } i=1,2
$$

after noting that

$$
\sup _{\epsilon>0} \sup _{\mathbf{x} \in \Omega} \tau_{\epsilon}(\mathbf{x})<\infty
$$

Together, we get the boundedness of $\mathcal{L}^{\epsilon}$.

We will also need to study the operator $\mathcal{L}_{\epsilon}$ defined over the space $L^{p}\left(\Omega \times[0, T] ; \mathbb{R}^{d}\right)$. It is clear that using Lemma 2.9 , for $\mathbf{v} \in L^{p}\left(\Omega \times[0, T] ; \mathbb{R}^{d}\right)$, the function $\mathcal{L}_{\epsilon} \mathbf{v}(\mathbf{x}, t)$ is also in $L^{p}\left(\Omega \times[0, T] ; \mathbb{R}^{d}\right)$. Moreover, repeating the exact argument as above, one can show that $\mathcal{L}_{\epsilon}$ is a uniformly bounded linear operator on $L^{p}\left(\Omega \times[0, T] ; \mathbb{R}^{d}\right)$.

\subsection{Two scale limit equation}

As part of obtaining the two scale limit equation, let us discuss the two scale convergence of $\mathcal{L}_{\epsilon} \mathbf{u}^{\epsilon}$, for a given two scale convergent sequence $\mathbf{u}^{\epsilon}$. The theorem we plan to prove is the following.

Theorem 4.4. Assume that $\mathcal{L}_{\epsilon}$ is the peridynamic operator given above. Suppose also that $\mathbf{u}^{\epsilon} \in$ $C\left([0, T] ; L^{p}\left(\Omega ; \mathbb{R}^{d}\right)\right.$ is a bounded sequence that two scale converges to $\mathbf{u} \in L^{p}\left(\Omega \times Y \times[0, T] ; \mathbb{R}^{d}\right)$. Then there exist bounded linear $\mathcal{L}_{0}$ on $L^{p}\left(\Omega \times Y \times[0, T] ; \mathbb{R}^{d}\right)$ and a subsequence $\epsilon_{n}$ such that as $n \rightarrow \infty$

$$
\mathcal{L}_{\epsilon_{n}} \mathbf{u}^{\epsilon_{n}} \stackrel{2}{\rightarrow} \mathcal{L}_{0} \mathbf{u}
$$

in $L^{p}\left(\Omega \times Y \times[0, T] ; \mathbb{R}^{d}\right)$.

For simplification, let us introduce a few notations corresponding to the kernel $\rho_{0}\left(\mathbf{x}^{\prime}-\mathbf{x}\right)$, we let

$$
\begin{gathered}
\lambda_{0}\left(\mathbf{x}^{\prime}-\mathbf{x}\right)=\rho_{0}\left(\mathbf{x}^{\prime}-\mathbf{x}\right)\left(\mathbf{x}^{\prime}-\mathbf{x}\right), \quad \Lambda(\mathbf{x})=\int_{\Omega} \boldsymbol{\lambda}_{0}\left(\mathbf{x}^{\prime}-\mathbf{x}\right) \mathrm{d} \mathbf{x}^{\prime} \\
m_{0}(\mathbf{x})=\int_{\Omega} \rho_{0}\left(\mathbf{x}^{\prime}-\mathbf{x}\right)\left|\mathbf{x}^{\prime}-\mathbf{x}\right|^{2} \mathrm{~d} \mathbf{x}^{\prime}
\end{gathered}
$$

The following is an elementary result that will be used to find the two scale limit of $\mathcal{L}_{\epsilon} \mathbf{u}^{\epsilon}$.

Lemma 4.5. Suppose that the bounded sequence $\mathbf{u}^{\epsilon}(\mathbf{x}, t)$ two scale converges to $\mathbf{u}(\mathbf{x}, \mathbf{y}, t)$ in $L^{p}(\Omega \times Y \times$ $\left.[0, T] ; \mathbb{R}^{d}\right)$. Suppose also that $\alpha, k$ are in $C_{\mathrm{per}}(Y)$. Then we have the following. As $\epsilon \rightarrow 0$,

(1) the sequence $\alpha_{\epsilon} \mathbf{u}^{\epsilon} \stackrel{2}{\rightarrow} \alpha(\mathbf{y}) \mathbf{u}(\mathbf{x}, \mathbf{y}, t)$ in $L^{p}\left(\Omega \times Y \times[0, T] ; \mathbb{R}^{k}\right)$; 
(2) the sequence $\tau_{\epsilon} \stackrel{2}{\rightarrow} \tau(\mathbf{x}, \mathbf{y})$ where $\tau(\mathbf{x}, \mathbf{y}):=\frac{d^{2} k(\mathbf{y})}{m_{0}(\mathbf{x})^{2}}-\frac{\alpha(\mathbf{y})}{m_{0}(\mathbf{x})}$ in $L^{p}(\Omega \times Y)$. Moreover, $\tau_{\epsilon} \rightarrow \bar{\tau}(\mathbf{x})$ weakly in $L^{p}(\Omega)$ with

$$
\bar{\tau}(\mathbf{x}):=\frac{d^{2}\langle k\rangle}{m_{0}(\mathbf{x})^{2}}-\frac{\langle\alpha\rangle}{m_{0}(\mathbf{x})}=\langle\tau((\mathbf{x}, \mathbf{y})\rangle
$$

and

$$
\tau_{\epsilon} \mathbf{u}^{\epsilon} \stackrel{2}{\rightarrow} \tau(\mathbf{x}, \mathbf{y}) \mathbf{u}(\mathbf{x}, \mathbf{y}, t) \quad \text { in } L^{p}\left(\Omega \times[0, T] \times Y ; \mathbb{R}^{k}\right) .
$$

Proof. We only proof part (2) of the lemma. Let us define

$$
\tau_{\epsilon}^{0}(\mathbf{x})=\frac{d^{2} k_{\epsilon}(\mathbf{x})}{m_{0}(\mathbf{x})^{2}}-\frac{\alpha_{\epsilon}(\mathbf{x})}{m_{0}(\mathbf{x})}
$$

Then noting that for all $\epsilon>0$, for all $\mathbf{x} \in \Omega, m_{\epsilon}(\mathbf{x}) \geq m_{0}(\mathbf{x}) \geq \min _{\mathbf{x} \in \Omega} m_{0}(\mathbf{x})>0$, and $\alpha$ and $k$ are bounded functions, it follows that there exists a constant $C>0$ such that for all $\epsilon>0$

$$
\left\|\tau_{\epsilon}-\tau_{\epsilon}^{0}\right\|_{L^{\infty}(\Omega)} \leq C\left(\left\|m_{\epsilon}^{2}-m_{0}^{2}\right\|_{L^{\infty}}+\left\|m_{\epsilon}-m_{0}\right\|_{L^{\infty}}\right)
$$

and therefore $\left\|\tau_{\epsilon}-\tau_{\epsilon}^{0}\right\|_{L^{\infty}(\Omega)} \rightarrow 0$, as $\epsilon \rightarrow 0$. As a consequence $\tau_{\epsilon}$ and $\tau_{\epsilon}^{0}$ will have the same two scale limit. We now use the fact that $\alpha_{\epsilon} \stackrel{2}{\rightarrow} \alpha(\mathbf{y})$ and $k_{\epsilon} \stackrel{2}{\rightarrow} k(\mathbf{y})$ to conclude that $\tau_{\epsilon}^{0} \stackrel{2}{\rightarrow} \tau(\mathbf{x}, \mathbf{y})$ as claimed.

Next, similar to the steps taken in [24], we introduce an auxiliary operator $\mathcal{L}_{b s}^{\epsilon}$ given by

$$
\begin{aligned}
\left(\mathcal{L}_{b s}^{\epsilon} \mathbf{v}\right)(\mathbf{x}, t):= & \int_{\Omega}\left(\alpha_{\epsilon}(\mathbf{x})+\alpha_{\epsilon}\left(\mathbf{x}^{\prime}\right)\right) \frac{\rho_{\epsilon}\left(\mathbf{x}, \mathbf{x}^{\prime}-\mathbf{x}\right)}{\left|\mathbf{x}^{\prime}-\mathbf{x}\right|^{2}}\left(\mathbf{x}^{\prime}-\mathbf{x}\right) \otimes\left(\mathbf{x}^{\prime}-\mathbf{x}\right)\left(\mathbf{v}\left(\mathbf{x}^{\prime}, t\right)-\mathbf{v}(\mathbf{x}, t)\right) \mathrm{d} \mathbf{x}^{\prime} \\
& +\int_{\Omega} \tau_{\epsilon}\left(\mathbf{x}^{\prime}\right) \boldsymbol{\lambda}_{0}\left(\mathbf{x}^{\prime}-\mathbf{x}\right)\left(\int_{\Omega} \boldsymbol{\lambda}_{0}\left(\mathbf{z}-\mathbf{x}^{\prime}\right) \cdot\left(\mathbf{v}(\mathbf{z}, t)-\mathbf{v}\left(\mathbf{x}^{\prime}, t\right)\right) \mathrm{d} \mathbf{z}\right) \mathrm{d} \mathbf{x}^{\prime} \\
& +\tau_{\epsilon}(\mathbf{x}) \Lambda(\mathbf{x}) \int_{\Omega} \boldsymbol{\lambda}_{0}(\mathbf{z}-\mathbf{x}) \cdot(\mathbf{v}(\mathbf{z}, t)-\mathbf{v}(\mathbf{x}, t)) \mathrm{d} \mathbf{z}
\end{aligned}
$$

which is, as we prove below, is the first order approximation of $\mathcal{L}_{\epsilon}$. The uniform boundedness of $\mathcal{L}_{b s}^{\epsilon}$ and the estimate on $\mathcal{L}_{\epsilon}-\mathcal{L}_{b s}^{\epsilon}$ given below again extend related results in the $L^{2}$ case proved in [24].

Theorem 4.6. $\mathcal{L}_{b s}^{\epsilon}$ is a bounded linear operator on $L^{p}\left(\Omega \times[0, T], \mathbb{R}^{d}\right)$. Moreover, the difference of the operators $\mathcal{L}_{\epsilon}$ and $\mathcal{L}_{b s}^{\epsilon}, \mathcal{L}_{\epsilon}-\mathcal{L}_{b s}^{\epsilon} \rightarrow 0$ in the operator norm, as $\epsilon \rightarrow 0$.

Proof. For any $\mathbf{u} \in L^{p}\left(\Omega \times[0, T] ; \mathbb{R}^{d}\right)$, the difference of the operator values $\left(\mathcal{L}_{\epsilon}-\mathcal{L}_{b s}^{\epsilon}\right) \mathbf{u}$ can be written as

$$
\left(\mathcal{L}_{\epsilon}-\mathcal{L}_{b s}^{\epsilon}\right) \mathbf{u}=J_{1}^{\epsilon} \mathbf{u}+J_{2}^{\epsilon} \mathbf{u}+J_{3}^{\epsilon} \mathbf{u}+J_{4}^{\epsilon} \mathbf{u}+J_{5}^{\epsilon} \mathbf{u}+J_{6}^{\epsilon} \mathbf{u}
$$

where using the notation $\boldsymbol{\lambda}_{2}(\boldsymbol{\xi})=\rho_{2}(\boldsymbol{\xi}) \boldsymbol{\xi}$, and the scaling $\boldsymbol{\lambda}_{2}^{\epsilon}(\boldsymbol{\xi})=\frac{1}{\epsilon^{d}} \boldsymbol{\lambda}_{2}\left(\frac{\boldsymbol{\xi}}{\epsilon}\right)$

$$
\begin{gathered}
J_{1}^{\epsilon} \mathbf{u}(\mathbf{x}, t)=\int_{\Omega} \tau_{\epsilon}\left(\mathbf{x}^{\prime}\right) \boldsymbol{\lambda}_{0}\left(\mathbf{x}^{\prime}-\mathbf{x}\right)\left(\int_{\Omega}\left(\beta\left(\frac{\mathbf{x}^{\prime}}{\epsilon}, \frac{\mathbf{z}}{\epsilon}\right) \boldsymbol{\lambda}_{2}^{\epsilon}\left(\mathbf{z}-\mathbf{x}^{\prime}\right) \cdot\left(\mathbf{u}(\mathbf{z}, t)-\mathbf{u}\left(\mathbf{x}^{\prime}, t\right)\right) \mathrm{d} \mathbf{z}\right) \mathrm{d} \mathbf{x}^{\prime}\right. \\
J_{2}^{\epsilon} \mathbf{u}(\mathbf{x}, t)=\int_{\Omega} \tau_{\epsilon}\left(\mathbf{x}^{\prime}\right)\left(\beta\left(\frac{\mathbf{x}}{\epsilon}, \frac{\mathbf{x}^{\prime}}{\epsilon}\right) \boldsymbol{\lambda}_{2}^{\epsilon}\left(\mathbf{x}^{\prime}-\mathbf{x}\right)\left(\int_{\Omega} \boldsymbol{\lambda}_{0}\left(\mathbf{z}-\mathbf{x}^{\prime}\right) \cdot\left(\mathbf{u}(\mathbf{z}, t)-\mathbf{u}\left(\mathbf{x}^{\prime}, t\right)\right) \mathrm{d} \mathbf{z}\right) \mathrm{d} \mathbf{x}^{\prime}\right. \\
J_{3}^{\epsilon} \mathbf{u}(\mathbf{x}, t)=\int_{\Omega} \tau_{\epsilon}\left(\mathbf{x}^{\prime}\right) \beta\left(\frac{\mathbf{x}}{\epsilon}, \frac{\mathbf{x}^{\prime}}{\epsilon}\right) \boldsymbol{\lambda}_{2}^{\epsilon}\left(\mathbf{x}^{\prime}-\mathbf{x}\right)\left(\int_{\Omega}\left(\beta\left(\frac{\mathbf{x}^{\prime}}{\epsilon}, \frac{\mathbf{z}}{\epsilon}\right) \boldsymbol{\lambda}_{2}^{\epsilon}\left(\mathbf{z}-\mathbf{x}^{\prime}\right) \cdot\left(\mathbf{u}(\mathbf{z}, t)-\mathbf{u}\left(\mathbf{x}^{\prime}, t\right)\right) \mathrm{d} \mathbf{z}\right) \mathrm{d} \mathbf{x}^{\prime}\right.
\end{gathered}
$$




$$
\begin{gathered}
J_{4}^{\epsilon} \mathbf{u}(\mathbf{x}, t)=\tau_{\epsilon}(\mathbf{x}) \Lambda(\mathbf{x})\left(\int_{\Omega} \beta\left(\frac{\mathbf{x}}{\epsilon}, \frac{\mathbf{z}}{\epsilon}\right) \boldsymbol{\lambda}_{2}^{\epsilon}(\mathbf{z}-\mathbf{x}) \cdot(\mathbf{u}(\mathbf{z}, t)-\mathbf{u}(\mathbf{x}, t)) \mathrm{d} \mathbf{z}\right) \\
J_{5}^{\epsilon} \mathbf{u}(\mathbf{x}, t)=\left(\tau_{\epsilon}(\mathbf{x}) \int_{\Omega} \beta\left(\frac{\mathbf{x}}{\epsilon}, \frac{\mathbf{x}^{\prime}}{\epsilon}\right) \boldsymbol{\lambda}_{2}^{\epsilon}\left(\mathbf{x}^{\prime}-\mathbf{x}\right) \mathrm{d} \mathbf{x}^{\prime}\right)\left(\int_{\Omega} \boldsymbol{\lambda}_{0}(\mathbf{z}-\mathbf{x}) \cdot(\mathbf{u}(\mathbf{z}, t)-\mathbf{u}(\mathbf{x}, t)) \mathrm{d} \mathbf{z}\right) \\
J_{6}^{\epsilon} \mathbf{u}(\mathbf{x}, t)=\left(\tau_{\epsilon}(\mathbf{x}) \int_{\Omega} \beta\left(\frac{\mathbf{x}}{\epsilon}, \frac{\mathbf{x}^{\prime}}{\epsilon}\right) \boldsymbol{\lambda}_{2}^{\epsilon}\left(\mathbf{x}^{\prime}-\mathbf{x}\right) \mathrm{d} \mathbf{x}^{\prime}\right)\left(\int_{\Omega} \beta\left(\frac{\mathbf{x}}{\epsilon}, \frac{\mathbf{z}}{\epsilon}\right) \boldsymbol{\lambda}_{2}^{\epsilon}(\mathbf{z}-\mathbf{x}) \cdot(\mathbf{u}(\mathbf{z}, t)-\mathbf{u}(\mathbf{x}, t)) \mathrm{d} \mathbf{z}\right) .
\end{gathered}
$$

Using Lemma 2.9 , it is not difficult to prove that $J_{i}^{\epsilon}$, for $i=1, \ldots, 6$ are bounded linear operators on $L^{p}\left(\Omega \times[0, T] ; \mathbb{R}^{d}\right)$. Moreover, the operator norm of each of these operators goes to 0 as $\epsilon \rightarrow 0$. In fact, after simple estimating we obtain that

$$
\sup _{\mathbf{u} \in L^{p}\left(\Omega \times[0, T] ; \mathbb{R}^{d}\right)} \frac{\left\|J_{i}^{\epsilon} \mathbf{u}\right\|_{L^{p}}}{\|\mathbf{u}\|_{L^{p}}}=\left\{\begin{array}{rl}
C \epsilon & \text { if } i=1,2,4,5 \\
C \epsilon^{2} & \text { if } i=3,6
\end{array},\right.
$$

for a constant $C$ independent of $\epsilon$.

The following lemma introduces the "limiting" operator $\mathcal{L}_{0}$ and whose boundedness and linearity is not difficult to prove.

Lemma 4.7. Define the operator $\mathcal{L}_{0}$ as

$$
\begin{aligned}
\left(\mathcal{L}_{0} \mathbf{u}\right)(\mathbf{x}, \mathbf{y})= & \alpha(\mathbf{y}) \int_{\Omega} \mathbf{K}_{0}\left(\mathbf{x}^{\prime}-\mathbf{x}\right)\left(\langle\mathbf{u}\rangle\left(\mathbf{x}^{\prime}\right)-\mathbf{u}(\mathbf{x}, \mathbf{y})\right) \mathrm{d} \mathbf{x}^{\prime} \\
& +\int_{\Omega} \mathbf{K}_{0}\left(\mathbf{x}^{\prime}-\mathbf{x}\right)\left(\langle\alpha \mathbf{u}\rangle\left(\mathbf{x}^{\prime}\right)-\langle\alpha\rangle \mathbf{u}(\mathbf{x}, \mathbf{y})\right) \mathrm{d} \mathbf{x}^{\prime} \\
& +\int_{\Omega} \boldsymbol{\lambda}\left(\mathbf{x}^{\prime}-\mathbf{x}\right) \int_{\Omega} \boldsymbol{\lambda}_{0}\left(\mathbf{z}-\mathbf{x}^{\prime}\right) \cdot\left(\bar{\tau}\left(\mathbf{x}^{\prime}\right)\langle\mathbf{u}\rangle(\mathbf{z})-\langle\tau \mathbf{u}\rangle\left(\mathbf{x}^{\prime}\right)\right) \mathrm{d} \mathbf{z} \mathrm{d} \mathbf{x}^{\prime} \\
& +\tau(\mathbf{x}, \mathbf{y}) \Lambda(\mathbf{x}) \int_{\Omega} \boldsymbol{\lambda}(\mathbf{z}-\mathbf{x}) \cdot(\langle\mathbf{u}\rangle(\mathbf{z})-\mathbf{u}(\mathbf{x}, \mathbf{y})) \mathrm{d} \mathbf{z} \\
& +\int_{B(\mathbf{y}, \delta)}\left(\alpha(\mathbf{y})+\alpha\left(\mathbf{y}^{\prime}\right)\right) \beta\left(\mathbf{y}, \mathbf{y}^{\prime}\right) \mathbf{K}_{2}\left(\mathbf{y}^{\prime}-\mathbf{y}\right)\left(\mathbf{u}\left(\mathbf{x}, \mathbf{y}^{\prime}\right)-\mathbf{u}(\mathbf{x}, \mathbf{y})\right) \mathrm{d} \mathbf{y}^{\prime}
\end{aligned}
$$

where $\mathbf{K}_{i}(\mathbf{z})=\frac{\rho_{i}(\mathbf{z})}{|\mathbf{z}|^{2}}(\mathbf{z} \otimes \mathbf{z})$ for $i=0,2$. Then $\mathcal{L}_{0}$ is a bounded linear operator on $L^{p}\left(\Omega \times Y ; \mathbb{R}^{d}\right)$. As a consequence, $\mathfrak{p}^{-1} \mathcal{L}_{0}$ is also a bounded operator on $L^{p}\left(\Omega \times Y ; \mathbb{R}^{d}\right)$.

Remark 4.8. From the definition of operator $\mathcal{L}_{0}$ and Lemma 2.9, if $\mathbf{u} \in L^{p}\left(\Omega \times Y \times[0, T] ; \mathbb{R}^{d}\right)$, then $\left(\mathcal{L}_{0} \mathbf{u}\right)(\mathbf{x}, \mathbf{y}, t) \in L^{p}\left(\Omega \times Y \times[0, T] ; \mathbb{R}^{d}\right)$. Moreover, $\mathcal{L}_{0}$ is also a bounded linear operator on $L^{p}\left(\Omega \times Y \times[0, T] ; \mathbb{R}^{d}\right)$.

Proof of Theorem 4.4. Suppose that $\mathbf{u}_{\epsilon} \stackrel{2}{\rightarrow} \mathbf{u}$ in $L^{p}\left(\Omega \times Y \times[0, T] ; \mathbb{R}^{d}\right)$. From Theorem 4.6, $\mathcal{L}_{\epsilon} \mathbf{u}_{\epsilon}$ and $\mathcal{L}_{b s}^{\epsilon} \mathbf{u}_{\epsilon}$ have the same two scale limit. Thus it suffices to find the two scale limit of the latter. The derivation of the two scale limit rests on Lemmas 4.5, and 2.10, the fact that $\mathcal{L}_{b s}^{\epsilon} \mathbf{u}_{\epsilon}$ is a finite sum of convolution-type operators and the convolution is a compact operator. We first write

$$
\mathcal{L}_{b s}^{\epsilon} \mathbf{u}_{\epsilon}=\mathcal{L}_{b s}^{\epsilon, 1} \mathbf{u}_{\epsilon}+\mathcal{L}_{b s}^{\epsilon, 2} \mathbf{u}_{\epsilon}+\mathcal{L}_{b s}^{\epsilon, 3}+\mathcal{L}_{b s}^{\epsilon, 4} \mathbf{u}_{\epsilon}
$$


and we will compute the two scale limit of each of the terms. First writing it as a sum of convolution type integrals, and then application of Lemma 4.5, Lemma 2.10 and the remark following it we see that

$$
\begin{aligned}
\mathcal{L}_{b s}^{\epsilon, 1} \mathbf{u}_{\epsilon}(\mathbf{x}, t):= & \int_{\Omega}\left(\alpha_{\epsilon}(\mathbf{x})+\alpha_{\epsilon}\left(\mathbf{x}^{\prime}\right)\right) \mathbf{K}_{0}\left(\mathbf{x}^{\prime}-\mathbf{x}\right)\left(\mathbf{u}^{\epsilon}\left(\mathbf{x}^{\prime}, t\right)-\mathbf{u}^{\epsilon}(\mathbf{x}, t)\right) \mathrm{d} \mathbf{x}^{\prime} \\
& \stackrel{2}{\rightarrow} \alpha(\mathbf{y}) \int_{\Omega} \frac{\rho_{0}\left(\mathbf{x}^{\prime}-\mathbf{x}\right)}{\left|\mathbf{x}^{\prime}-\mathbf{x}\right|^{2}}\left(\mathbf{x}^{\prime}-\mathbf{x}\right) \otimes\left(\mathbf{x}^{\prime}-\mathbf{x}\right)\left(\langle\mathbf{u}\rangle\left(\mathbf{x}^{\prime}, t\right)-\mathbf{u}(\mathbf{x}, \mathbf{y}, t)\right) \mathrm{d} \mathbf{x}^{\prime} \\
& +\int_{\Omega} \frac{\rho_{0}\left(\mathbf{x}^{\prime}-\mathbf{x}\right)}{\left|\mathbf{x}^{\prime}-\mathbf{x}\right|^{2}}\left(\mathbf{x}^{\prime}-\mathbf{x}\right) \otimes\left(\mathbf{x}^{\prime}-\mathbf{x}\right)\left(\langle\alpha \mathbf{u}\rangle\left(\mathbf{x}^{\prime}, t\right)-\langle\alpha\rangle \mathbf{u}(\mathbf{x}, \mathbf{y}, t)\right) \mathrm{d} \mathbf{x}^{\prime} .
\end{aligned}
$$

Again with a similar approach the two scale limit of $\mathcal{L}_{b s}^{\epsilon, 2} \mathbf{u}_{\epsilon}$ is computed as

$$
\begin{aligned}
\mathcal{L}_{b s}^{\epsilon, 2} \mathbf{u}_{\epsilon}(\mathbf{x}, t):= & \int_{\Omega}\left(\alpha_{\epsilon}(\mathbf{x})+\alpha_{\epsilon}\left(\mathbf{x}^{\prime}\right)\right) \beta\left(\frac{\mathbf{x}}{\epsilon}, \frac{\mathbf{x}^{\prime}}{\epsilon}\right) \frac{1}{\epsilon^{d}} \mathbf{K}_{2}\left(\frac{\mathbf{x}^{\prime}-\mathbf{x}}{\epsilon}\right)\left(\mathbf{u}^{\epsilon}\left(\mathbf{x}^{\prime}, t\right)-\mathbf{u}^{\epsilon}(\mathbf{x}, t)\right) \mathrm{d} \mathbf{x}^{\prime} \\
& \stackrel{2}{\longrightarrow} \int_{B(\mathbf{y}, \delta)}\left(\alpha(\mathbf{y})+\alpha\left(\mathbf{y}^{\prime}\right)\right) \beta\left(\mathbf{y}, \mathbf{y}^{\prime}\right) \mathbf{K}_{2}\left(\mathbf{y}^{\prime}-\mathbf{y}\right)\left(\mathbf{u}\left(\mathbf{x}, \mathbf{y}^{\prime}, t\right)-\mathbf{u}(\mathbf{x}, \mathbf{y}, t)\right) \mathrm{d} \mathbf{y}^{\prime}
\end{aligned}
$$

Indeed, write $\mathcal{L}_{b s}^{\epsilon, 2} \mathbf{u}_{\epsilon}(\mathbf{x}, t)=I_{1}^{\epsilon} \mathbf{u}_{\epsilon}(\mathbf{x}, t)-I_{2}^{\epsilon} \mathbf{u}_{\epsilon}(\mathbf{x}, t)$, where

$$
I_{1}^{\epsilon} \mathbf{u}_{\epsilon}(\mathbf{x}, t)=\int_{\Omega}\left(\alpha_{\epsilon}(\mathbf{x})+\alpha_{\epsilon}\left(\mathbf{x}^{\prime}\right)\right) \beta\left(\frac{\mathbf{x}}{\epsilon}, \frac{\mathbf{x}^{\prime}}{\epsilon}\right) \frac{\rho_{2}^{\epsilon}\left(\mathbf{x}^{\prime}-\mathbf{x}\right)}{\left|\mathbf{x}^{\prime}-\mathbf{x}\right|^{2}}\left(\mathbf{x}^{\prime}-\mathbf{x}\right) \otimes\left(\mathbf{x}^{\prime}-\mathbf{x}\right) \mathbf{u}_{\epsilon}\left(\mathbf{x}^{\prime}, t\right) \mathrm{d} \mathbf{x}^{\prime}
$$

and

$$
I_{2}^{\epsilon} \mathbf{u}_{\epsilon}(\mathbf{x}, t)=\int_{\Omega}\left(\alpha_{\epsilon}(\mathbf{x})+\alpha_{\epsilon}\left(\mathbf{x}^{\prime}\right)\right) \beta\left(\frac{\mathbf{x}}{\epsilon}, \frac{\mathbf{x}^{\prime}}{\epsilon}\right) \frac{\rho_{2}^{\epsilon}\left(\mathbf{x}^{\prime}-\mathbf{x}\right)}{\left|\mathbf{x}^{\prime}-\mathbf{x}\right|^{2}}\left(\mathbf{x}^{\prime}-\mathbf{x}\right) \otimes\left(\mathbf{x}^{\prime}-\mathbf{x}\right) \mathrm{d} \mathbf{x}^{\prime} \mathbf{u}_{\epsilon}(\mathbf{x}, t) .
$$

We will find the two scale limit of each of these terms. Let us begin with $I_{1}^{\epsilon} \mathbf{u}_{\epsilon}$. For each $\mathbf{x}$, after making the change of variables $\mathbf{x}^{\prime}=\mathbf{x}+\epsilon \mathbf{z}$, we have

$$
I_{1}^{\epsilon} \mathbf{u}_{\epsilon}(\mathbf{x}, t)=\int_{B(0, \delta)}\left(\alpha\left(\frac{\mathbf{x}}{\epsilon}\right)+\alpha\left(\frac{\mathbf{x}}{\epsilon}+\mathbf{z}\right)\right) \beta\left(\frac{\mathbf{x}}{\epsilon}, \frac{\mathbf{x}}{\epsilon}+\mathbf{z}\right) \mathbf{K}_{2}(\mathbf{z}) \overline{\mathbf{u}}_{\epsilon}(\mathbf{x}+\epsilon \mathbf{z}, t) \mathrm{d} \mathbf{z} .
$$

Now suppose that $\boldsymbol{\psi}(\mathbf{x}, \mathbf{y}, t)$, is a given smooth test function. Then for each $\mathbf{z}, \boldsymbol{\Psi}(\mathbf{x}, \mathbf{y}):=(\alpha(\mathbf{y})+\alpha(\mathbf{y}+$ $\mathbf{z})) \beta(\mathbf{y}, \mathbf{y}+\mathbf{z}) \boldsymbol{\psi}(\mathbf{x}, \mathbf{y}, t)$ is also an admissible test function. Then we have that

$$
\int_{0}^{T} \int_{\Omega} I_{1}^{\epsilon} \mathbf{u}_{\epsilon}(\mathbf{x}, t) \cdot \psi(\mathbf{x}, \mathbf{x} / \epsilon, t) \mathrm{d} \mathbf{x} \mathrm{d} t=\int_{B(0, \delta)} \frac{\rho_{2}(\mathbf{z})}{|\mathbf{z}|^{2}}\left(\int_{0}^{T} \int_{\Omega} \mathbf{z} \cdot \overline{\mathbf{u}}_{\epsilon}(\mathbf{x}+\epsilon \mathbf{z}, t) \mathbf{z} \cdot \boldsymbol{\Psi}\left(\mathbf{x}, \frac{\mathbf{x}}{\epsilon}, t\right) \mathrm{d} \mathbf{x} \mathrm{d} t\right) \mathrm{d} \mathbf{z} .
$$

Denoting the inner integral by $Q^{\epsilon}(\mathbf{z})$, and noting that $\overline{\mathbf{u}}_{\epsilon}(\mathbf{x}+\epsilon \mathbf{z}, t) \stackrel{2}{\rightarrow} \mathbf{u}(\mathbf{x}, \mathbf{y}+\mathbf{z}, t)$ and using $\boldsymbol{\Psi}$ as a test function, it follows from the two scale convergence of $\mathbf{u}^{\epsilon}$ that for each $\mathbf{z}$,

$$
Q^{\epsilon}(\mathbf{z}) \rightarrow \int_{0}^{T} \int_{\Omega \times Y}(\alpha(\mathbf{y})+\alpha(\mathbf{y}+\mathbf{z})) \beta(\mathbf{y}, \mathbf{z}+z) \mathbf{z} \cdot \mathbf{u}(\mathbf{x}, \mathbf{y}+\mathbf{z}, t) \mathbf{z} \cdot \boldsymbol{\psi}(\mathbf{x}, \mathbf{y}, t) \mathrm{d} \mathbf{y} \mathrm{d} \mathbf{x} \mathrm{d} t .
$$

Also note that for each $\mathbf{z},\left|Q^{\epsilon}(\mathbf{z})\right| \leq C\left\|\mathbf{u}_{\epsilon}\right\|_{L^{p}} \leq C$. Then applying uniform bounded convergence theorem, it follows that as $\epsilon \rightarrow 0$,

$$
\begin{aligned}
& \int_{0}^{T} \int_{\Omega} I_{1}^{\epsilon} \mathbf{u}_{\epsilon}(\mathbf{x}, t) \cdot \psi(\mathbf{x}, \mathbf{x} / \epsilon, t) \mathrm{d} \mathbf{x} \mathrm{d} t=\int_{B(0, \delta)} \frac{\rho_{2}(\mathbf{z})}{|\mathbf{z}|^{2}} Q^{\epsilon}(\mathbf{z}) \mathrm{d} \mathbf{z} \\
& \rightarrow \int_{B(0, \delta)} \frac{\rho_{2}(\mathbf{z})}{|\mathbf{z}|^{2}} \int_{0}^{T} \int_{\Omega \times Y}(\alpha(\mathbf{y})+\alpha(\mathbf{y}+\mathbf{z})) \beta(\mathbf{y}, \mathbf{y}+z) \mathbf{z} \cdot \mathbf{u}(\mathbf{x}, \mathbf{y}+\mathbf{z}, t) \mathbf{z} \cdot \psi(\mathbf{x}, \mathbf{y}, t) \mathrm{d} \mathbf{y} \mathrm{d} \mathbf{x} \mathrm{d} t \mathrm{~d} \mathbf{z}
\end{aligned}
$$


Rewriting the last limit we observe that

$$
\begin{aligned}
& \lim _{\epsilon \rightarrow 0} \int_{0}^{T} \int_{\Omega} I_{1}^{\epsilon} \mathbf{u}_{\epsilon}(\mathbf{x}, t) \cdot \psi(\mathbf{x}, \mathbf{x} / \epsilon, t) \mathrm{d} \mathbf{x} \mathrm{d} t \\
& =\int_{0}^{T} \int_{\Omega \times Y}\left(\int_{B(\mathbf{y}, \delta)}\left(\alpha(\mathbf{y})+\alpha\left(\mathbf{y}^{\prime}\right)\right) \beta\left(\mathbf{y}, \mathbf{y}^{\prime}\right) \mathbf{K}_{2}\left(\mathbf{y}^{\prime}-\mathbf{y}\right) \mathbf{u}\left(\mathbf{x}, \mathbf{y}^{\prime}, t\right) \mathrm{d} \mathbf{y}^{\prime}\right) \cdot \boldsymbol{\psi}(\mathbf{x}, \mathbf{y}, t) \mathrm{d} \mathbf{y} \mathrm{d} \mathbf{x} \mathrm{d} t .
\end{aligned}
$$

To find the two scale limit of $I_{2}^{\epsilon} \mathbf{u}_{\epsilon}$, we first observe after change of variables that

$$
I_{2}^{\epsilon} \mathbf{u}_{\epsilon}(\mathbf{x}, t)=\left(\varphi\left(\frac{\mathbf{x}}{\epsilon}\right)+\mathbf{f}_{\epsilon}(\mathbf{x})\right) \mathbf{u}^{\epsilon}(\mathbf{x}, t)
$$

where $\varphi(\mathbf{y})$ is a $Y$-periodic continuous matrix function given by change variables to obtain that

$$
\boldsymbol{\varphi}(\mathbf{y})=\int_{B(0, \delta)}(\alpha(\mathbf{y})+\alpha(\mathbf{y}+\mathbf{z})) \beta(\mathbf{y}, \mathbf{y}+\mathbf{z}) \mathbf{K}_{2}(\mathbf{z}) \mathrm{d} \mathbf{z}
$$

and for any $\mathbf{x} \in \Omega$

$$
\mathbf{f}_{\epsilon}(\mathbf{x})=\int_{B(0, \delta)}(\chi(\mathbf{x}+\epsilon \mathbf{z})-1)(\alpha(\mathbf{y})+\alpha(\mathbf{y}+\mathbf{z})) \beta(\mathbf{y}, \mathbf{y}+\mathbf{z}) \mathbf{K}_{2}(\mathbf{z}) \mathrm{d} \mathbf{z} .
$$

Clearly $\mathbf{f}_{\epsilon}(\mathbf{x}) \rightarrow 0$ strongly in $L^{p}(\Omega)$ for any $p>1$, and therefore, $I_{2}^{\epsilon} \mathbf{u}^{\epsilon}(\mathbf{x}, t)$ and $\boldsymbol{\varphi}\left(\frac{\mathbf{x}}{\epsilon}\right) \mathbf{u}^{\epsilon}$ have the same two scale limit. We now apply Lemma 4.5 to prove that as $\epsilon \rightarrow 0$,

$$
\boldsymbol{\varphi}\left(\frac{\mathbf{x}}{\epsilon}\right) \mathbf{u}^{\epsilon} \stackrel{2}{\rightarrow} \varphi(\mathbf{y}) \mathbf{u}(\mathbf{x}, \mathbf{y}, t) \text { in } L^{p}\left(\Omega \times Y \times[0, T] ; \mathbb{R}^{d}\right)
$$

We can then conclude that as $\epsilon \rightarrow 0$,

$$
I_{2}^{\epsilon} \mathbf{u}^{\epsilon}(\mathbf{x}, t) \stackrel{2}{\rightarrow} \int_{B(\mathbf{y}, \delta)}\left(\alpha(\mathbf{y})+\alpha\left(\mathbf{y}^{\prime}\right)\right) \beta\left(\mathbf{y}, \mathbf{y}^{\prime}\right) \mathbf{K}_{2}\left(\mathbf{y}^{\prime}-\mathbf{y}\right) \mathrm{d} \mathbf{y}^{\prime} \mathbf{u}(\mathbf{x}, \mathbf{y}, t) .
$$

Finally the two scale limit (up to a subsequence) of $\mathcal{L}_{b s}^{\epsilon, 3} \mathbf{u}^{\epsilon}$ and $\mathcal{L}_{b s}^{\epsilon, 4} \mathbf{u}^{\epsilon}$ can be computed as follows using Lemma 4.5 and Lemma 2.10

$$
\begin{aligned}
\mathcal{L}_{b s}^{\epsilon, 3} \mathbf{u}^{\epsilon}(\mathbf{x}, t):= & \int_{\Omega} \tau_{\epsilon}\left(\mathbf{x}^{\prime}\right) \boldsymbol{\lambda}_{0}\left(\mathbf{x}^{\prime}-\mathbf{x}\right)\left(\int_{\Omega} \boldsymbol{\lambda}_{1}\left(\mathbf{z}-\mathbf{x}^{\prime}\right) \cdot\left(\mathbf{u}^{\epsilon}(\mathbf{z}, t)-\mathbf{u}^{\epsilon}\left(\mathbf{x}^{\prime}, t\right)\right) \mathrm{d} \mathbf{z}\right) \mathrm{d} \mathbf{x}^{\prime} \\
& \stackrel{2}{\rightarrow} \int_{\Omega} \boldsymbol{\lambda}\left(\mathbf{x}^{\prime}-\mathbf{x}\right) \int_{\Omega} \boldsymbol{\lambda}_{0}\left(\mathbf{z}-\mathbf{x}^{\prime}\right) \cdot\left(\langle\tau\rangle\left(\mathbf{x}^{\prime}\right)\langle\mathbf{u}\rangle(\mathbf{z}, t)-\langle\tau \mathbf{u}\rangle\left(\mathbf{x}^{\prime}, t\right)\right) \mathrm{d} \mathbf{z} \mathrm{d} \mathbf{x}^{\prime}
\end{aligned}
$$

and

$$
\begin{aligned}
\mathcal{L}_{b s}^{\epsilon, 4} \mathbf{u}^{\epsilon}(\mathbf{x}, t) & :=\tau_{\epsilon}(\mathbf{x}) \Lambda(\mathbf{x}) \int_{\Omega} \boldsymbol{\lambda}_{0}(\mathbf{z}-\mathbf{x}) \cdot\left(\mathbf{u}^{\epsilon}(\mathbf{z}, t)-\mathbf{u}^{\epsilon}(\mathbf{x}, t)\right) \mathrm{d} \mathbf{z} \\
& \stackrel{2}{\longrightarrow} \tau(\mathbf{x}, \mathbf{y}) \Lambda(\mathbf{x}) \int_{\Omega} \boldsymbol{\lambda}(\mathbf{z}-\mathbf{x}) \cdot(\langle\mathbf{u}\rangle(\mathbf{z}, t)-\mathbf{u}(\mathbf{x}, \mathbf{y}, t)) \mathrm{d} \mathbf{z} .
\end{aligned}
$$

Finally we give the two scale limit equation of the heterogeneous peridynamic equaiton.

Theorem 4.9. Suppose that $\mathbf{u}^{\epsilon}(\mathbf{x}, t)$ is the solution to the peridynamics equation of motion (4.1) with the forcing term $\mathbf{b}^{\epsilon}$ two scale converge to $\mathbf{b}(\mathbf{x}, \mathbf{y}, t)$, the initial data $\mathbf{u}_{0}^{\epsilon}$, and $\mathbf{v}_{0}^{\epsilon}$ two scale converge in $L^{p}\left(\Omega \times Y ; \mathbb{R}^{d}\right)$ to $\mathbf{u}_{0}(\mathbf{x}, \mathbf{y})$ and $\mathbf{v}_{0}(\mathbf{x}, \mathbf{y})$ respectively. If $\mathcal{L}_{0}$ is the operator defined in Lemma 4.7, then $\mathbf{u}_{\epsilon} \stackrel{2}{\rightarrow} \mathbf{u}$ in $L^{p}(\Omega \times Y \times$ $\left.[0, T] ; \mathbb{R}^{d}\right)$ where $\mathbf{u}(\mathbf{x}, \mathbf{y}, t)$ uniquely solves the nonlocal system of linear equations

$$
\mathfrak{p}(\mathbf{y}) \partial_{t t} \mathbf{u}(\mathbf{x}, \mathbf{y}, t)=\left(\mathcal{L}_{0} \mathbf{u}\right)(\mathbf{x}, \mathbf{y}, t)+\mathbf{b}(\mathbf{x}, \mathbf{y}, t)
$$

with initial conditions

$$
\mathbf{u}(\mathbf{x}, \mathbf{y}, 0)=\mathbf{u}_{0}(\mathbf{x}, \mathbf{y}), \quad \partial_{t} \mathbf{u}(\mathbf{x}, \mathbf{y}, 0)=\mathbf{v}_{0}(\mathbf{x}, \mathbf{y})
$$


The proof of the theorem exactly parallels that of Theoem 3.1, where we make use of Lemma 2.14 and Theorem 4.4. We remark that given the linearity and the boundedness of the operator $\mathcal{L}_{0}$, the two scale limit $\mathbf{u}$ is uniquely defined by the equations (4.3)-(4.4). Moreover, if both the body force, $\mathbf{b}(\mathbf{x}, \mathbf{y}, t)$ and the initial data $\mathbf{u}_{0}(\mathbf{x}, \mathbf{y})$ and $\mathbf{v}_{0}(\mathbf{x}, \mathbf{y})$ are extended periodically with period $Y$, in the $\mathbf{y}$-variable to the whole of $\mathbb{R}^{d}$, then the periodically extended function $\mathbf{u}$ will still be a solution to (4.3)-(4.4). From here on we assume that this is the case.

\subsection{Homogenized peridynamics}

From Theorem 4.9 we obtain that the average value $\langle\mathbf{u}\rangle(\mathbf{x})$ of $\mathbf{u}(\mathbf{x}, \mathbf{y}, t)$, over period $Y$, approximates on average or the overall behavior of the actual field $\mathbf{u}^{\epsilon}$ solving (4.1). We also have and the weak convergence $\mathbf{u}_{\epsilon} \rightarrow\langle\mathbf{u}\rangle$, weakly in $L^{p}(\Omega \times[0, T])$, and that for any subdomain $V \subset \Omega$, and any interval $\left(t_{0}, t_{1}\right)$,

$$
\lim _{\epsilon \rightarrow 0} \int_{V \times\left(t_{0}, t_{1}\right)} \mathbf{u}^{\epsilon}(\mathbf{x}, t) \mathrm{d} \mathbf{x} \mathrm{d} t=\lim _{\epsilon \rightarrow 0} \int_{V \times\left(t_{0}, t_{1}\right)}\langle\mathbf{u}\rangle(\mathbf{x}, t) \mathrm{d} \mathbf{x} \mathrm{d} t .
$$

We may write the operator $\mathcal{L}_{0}$ given in $(4.2)$ as

$$
\left(\mathcal{L}_{0} \mathbf{u}\right)(\mathbf{x}, \mathbf{y})=\mathcal{L}_{\text {ave }}^{1}\langle\mathbf{u}\rangle(\mathbf{x})+\left(\mathcal{L}_{\text {osc }}^{1}\langle\mathbf{u}\rangle\right)(\mathbf{x}, \mathbf{y})+\mathcal{M}^{1}(\mathbf{u}-\langle\mathbf{u}\rangle)(\mathbf{x}, \mathbf{y})+\left(\mathcal{M}^{2} \mathbf{u}\right)(\mathbf{x}, \mathbf{y}),
$$

where the operators on the right hand side are defined by

$$
\begin{aligned}
\mathcal{L}_{\mathrm{ave}}^{1} \mathbf{u}^{H}(\mathbf{x})= & 2\langle\alpha\rangle \int_{\Omega} \frac{\rho_{0}\left(\mathbf{x}^{\prime}-\mathbf{x}\right)}{\left|\mathbf{x}^{\prime}-\mathbf{x}\right|^{2}}\left(\mathbf{x}^{\prime}-\mathbf{x}\right) \otimes\left(\mathbf{x}^{\prime}-\mathbf{x}\right)\left(\mathbf{u}^{H}\left(\mathbf{x}^{\prime}\right)-\mathbf{u}^{H}(\mathbf{x})\right) \mathrm{d} \mathbf{x}^{\prime} \\
& +\int_{\Omega}\langle\tau\rangle\left(\mathbf{x}^{\prime}\right) \boldsymbol{\lambda}_{0}\left(\mathbf{x}^{\prime}-\mathbf{x}\right) \int_{\Omega} \boldsymbol{\lambda}_{0}\left(\mathbf{z}-\mathbf{x}^{\prime}\right) \cdot\left(\mathbf{u}^{H}(\mathbf{z})-\mathbf{u}^{H}\left(\mathbf{x}^{\prime}\right)\right) \mathrm{d} \mathbf{z} \mathrm{d} \mathbf{x}^{\prime} \\
& +\langle\tau\rangle(\mathbf{x}) \Lambda(\mathbf{x}) \int_{\Omega} \boldsymbol{\lambda}_{0}(\mathbf{z}-\mathbf{x}) \cdot\left(\mathbf{u}^{H}(\mathbf{z})-\mathbf{u}^{H}(\mathbf{x})\right) \mathrm{d} \mathbf{z}, \\
\left(\mathcal{L}_{\mathrm{osc}}^{1} \mathbf{u}^{H}\right)(\mathbf{x}, \mathbf{y})= & (\alpha(\mathbf{y})-\langle\alpha\rangle) \int_{\Omega} \mathbf{K}_{0}\left(\mathbf{x}^{\prime}-\mathbf{x}\right)\left(\mathbf{u}^{H}\left(\mathbf{x}^{\prime}\right)-\mathbf{u}^{H}(\mathbf{x})\right) \mathrm{d} \mathbf{x}^{\prime} \\
& +(\tau(\mathbf{x}, \mathbf{y})-\langle\tau\rangle) \Lambda(\mathbf{x}) \int_{\Omega} \boldsymbol{\lambda}_{0}(\mathbf{z}-\mathbf{x}) \cdot\left(\mathbf{u}^{H}(\mathbf{z})-\mathbf{u}^{H}(\mathbf{x})\right) \mathrm{d} \mathbf{z}
\end{aligned}
$$

and

$$
\begin{aligned}
\left(\mathcal{M}^{1} \mathbf{w}\right)(\mathbf{x}, \mathbf{y})= & \int_{\Omega} \mathbf{K}_{0}\left(\mathbf{x}^{\prime}-\mathbf{x}\right)\langle\alpha \mathbf{w}\rangle\left(\mathbf{x}^{\prime}\right) \mathrm{d} \mathbf{x}^{\prime}-\int_{\Omega} \boldsymbol{\lambda}_{0}\left(\mathbf{x}^{\prime}-\mathbf{x}\right) \int_{\Omega} \boldsymbol{\lambda}_{0}\left(\mathbf{z}-\mathbf{x}^{\prime}\right) \cdot\langle\tau \mathbf{w}\rangle\left(\mathbf{x}^{\prime}\right) \mathrm{d} \mathbf{z} \mathrm{d} \mathbf{x}^{\prime} \\
& -(\alpha(\mathbf{y})+\langle\alpha\rangle) \mathbb{A}(\mathbf{x}) \mathbf{w}(\mathbf{x}, \mathbf{y})-\tau(\mathbf{x}, \mathbf{y}) \Lambda(\mathbf{x}) \otimes \Lambda(\mathbf{x})(\mathbf{w}(\mathbf{x}, \mathbf{y})) \\
= & \int_{\Omega} \mathbf{K}_{0}\left(\mathbf{x}^{\prime}-\mathbf{x}\right)\langle\alpha \mathbf{w}\rangle\left(\mathbf{x}^{\prime}\right) \mathrm{d} \mathbf{x}^{\prime}-\int_{\Omega} \boldsymbol{\lambda}_{0}\left(\mathbf{x}^{\prime}-\mathbf{x}\right) \Lambda\left(\mathbf{x}^{\prime}\right) \cdot\langle\tau \mathbf{w}\rangle\left(\mathbf{x}^{\prime}\right) \mathrm{d} \mathbf{x}^{\prime}-\mathbb{P}(\mathbf{x}, \mathbf{y})(\mathbf{w}(\mathbf{x}, \mathbf{y}))
\end{aligned}
$$

where

$$
\mathbb{A}(\mathbf{x})=\int_{\Omega} \mathbf{K}_{0}\left(\mathbf{x}^{\prime}-\mathbf{x}\right) \mathrm{d} \mathbf{x}^{\prime}
$$

and

$$
\mathbb{P}(\mathbf{x}, \mathbf{y})=(\alpha(\mathbf{y})+\langle\alpha\rangle) \mathbb{A}(\mathbf{x}))+\tau(\mathbf{x}, \mathbf{y}) \Lambda(\mathbf{x}) \otimes \Lambda(\mathbf{x}),
$$

is the two scale limit of $\mathbb{P}_{\epsilon}^{0}(\mathbf{x})$ which is defined as in [24] by,

$$
\mathbb{P}_{\epsilon}^{0}(\mathbf{x})=\int_{\Omega}\left(\alpha_{\epsilon}(\mathbf{x})+\alpha_{\epsilon}\left(\mathbf{x}^{\prime}\right)\right) \mathbf{K}_{0}\left(\mathbf{x}^{\prime}-\mathbf{x}\right) \mathrm{d} \mathbf{x}^{\prime}+\tau_{\epsilon}^{0}(\mathbf{x}) \Lambda(\mathbf{x}) \otimes \Lambda(\mathbf{x}) .
$$


$\mathbb{P}_{\epsilon}^{0}(\mathbf{x})$ is the so-called stability matrix and it has been shown to be uniformly positive definite [24]. And finally,

$$
\mathcal{M}^{2} \mathbf{w}(\mathbf{x}, \mathbf{y})=\int_{B(\mathbf{y}, \delta)}\left(\alpha(\mathbf{y})+\alpha\left(\mathbf{y}^{\prime}\right)\right) \beta\left(\mathbf{y}, \mathbf{y}^{\prime}\right) \mathbf{K}_{2}\left(\mathbf{y}^{\prime}-\mathbf{y}\right)\left(\mathbf{w}\left(\mathbf{x}, \mathbf{y}^{\prime}\right)-\mathbf{w}(\mathbf{x}, \mathbf{y})\right) \mathrm{d} \mathbf{y}^{\prime} .
$$

Defining $\mathbf{u}^{H}(\mathbf{x})=\langle\mathbf{u}\rangle(\mathbf{x})$, and write $\mathbf{u}(\mathbf{x}, \mathbf{y}, t)=\mathbf{u}^{H}(\mathbf{x}, t)+\mathbf{w}(\mathbf{x}, \mathbf{y}, t)$. Then $\mathbf{w} \in L^{p}\left(\Omega \times Y \times[0, T] ; \mathbb{R}^{d}\right)$ and $\langle\mathbf{w}\rangle(\mathbf{x}, t)=0$ for all $(\mathbf{x}, t) \in \Omega \times[0, T]$. We now present a means of obtaining the functions $\mathbf{u}^{H}$ and $\mathbf{w}(\mathbf{x}, \mathbf{y}, t)$. Notice that the two scale dynamic equation (4.3) can be expressed as

$$
\begin{aligned}
\mathbf{u}_{t t}^{H}+\mathbf{w}_{t t}= & \mathfrak{p}^{-1}(\mathbf{y}) \mathcal{L}_{\text {ave }}^{1} \mathbf{u}^{H}(\mathbf{x}, t)+\mathfrak{p}^{-1}(\mathbf{y})\left(\mathcal{L}_{\mathrm{osc}}^{1} \mathbf{u}^{H}\right)(\mathbf{x}, \mathbf{y}, t) \\
& +\mathfrak{p}^{-1}(\mathbf{y})(\mathcal{M} \mathbf{w})(\mathbf{x}, \mathbf{y}, t)+\mathfrak{p}^{-1}(\mathbf{y}) \mathbf{b}(\mathbf{x}, \mathbf{y}, t)
\end{aligned}
$$

where $(\mathcal{M} \mathbf{w})(\mathbf{x}, \mathbf{y})=\left(\mathcal{M}^{1} \mathbf{w}\right)(\mathbf{x}, \mathbf{y})+\mathcal{M}^{2} \mathbf{w}(\mathbf{x}, \mathbf{y})$. Integrating over $Y$ and using the fact that $\langle\mathbf{w}\rangle=0$, we obtain the equation for all $(\mathbf{x}, t) \in \Omega \times[0, T]$,

$$
\mathbf{u}_{t t}^{H}(\mathbf{x}, t)=\left\langle\mathfrak{p}^{-1}\right\rangle \mathcal{L}_{\text {ave }}^{1} \mathbf{u}^{H}(\mathbf{x}, t)+\left\langle\mathfrak{p}^{-1} \mathcal{L}_{\mathrm{osc}}^{1} \mathbf{u}^{H}\right\rangle(\mathbf{x}, t)+\left\langle\mathfrak{p}^{-1} \mathcal{M} \mathbf{w}\right\rangle(\mathbf{x}, t)+\left\langle\mathfrak{p}^{-1} \mathbf{b}\right\rangle(\mathbf{x}, t) .
$$

We now plug in (4.7) in (4.6), we obtain that for all $(\mathbf{x}, \mathbf{y}) \in \Omega \times Y$ and $t>0$

$$
\mathbf{w}_{t t}(\mathbf{x}, \mathbf{y}, t)=\left(\mathcal{P}_{\mathfrak{p}} \mathbf{u}^{H}\right)(\mathbf{x}, \mathbf{y}, t)+\mathcal{K}_{\mathfrak{p}} \mathbf{w}(\mathbf{x}, \mathbf{y}, t)+\mathbf{B}_{\mathfrak{p}}(\mathbf{x}, \mathbf{y}, t),
$$

where the operator

$$
\left(\mathcal{P}_{\mathfrak{p}} \mathbf{u}^{H}\right)(\mathbf{x}, \mathbf{y}, t)=\left(\mathfrak{p}^{-1}(\mathbf{y})-\left\langle\mathfrak{p}^{-1}\right\rangle\right) \mathcal{L}_{\text {ave }}^{1} \mathbf{u}^{H}(\mathbf{x}, t)+\mathfrak{p}^{-1}(\mathbf{y})\left(\mathcal{L}_{\text {osc }}^{1} \mathbf{u}^{H}\right)(\mathbf{x}, \mathbf{y}, t)-\left\langle\mathfrak{p}^{-1} \mathcal{L}_{\text {osc }}^{1} \mathbf{u}^{H}\right\rangle(\mathbf{x}, t)
$$

and

$$
\mathcal{K}_{\mathfrak{p}} \mathbf{w}(\mathbf{x}, \mathbf{y}, t):=\mathfrak{p}^{-1}(\mathbf{y}) \mathcal{L}_{0} \mathbf{w}(\mathbf{x}, \mathbf{y}, t)-\left\langle\mathfrak{p}^{-1} \mathcal{L}_{0} \mathbf{w}\right\rangle(\mathbf{x}, t),
$$

is defined over the set of functions $\mathbf{w}(\mathbf{x}, \mathbf{y}, t)$ such that $\langle\mathbf{w}\rangle=0$ and the function $\mathbf{B}_{\mathfrak{p}}(\mathbf{x}, \mathbf{y}, t):=\mathfrak{p}^{-1}(\mathbf{y}) \mathbf{b}(\mathbf{x}, \mathbf{y}, t)-$ $\left\langle\mathfrak{p}^{-1} \mathbf{b}\right\rangle(\mathbf{x}, t)$. The coupled system (4.7) and (4.8) together with the initial data

$$
\begin{aligned}
\mathbf{u}^{H}(\mathbf{x}, 0) & =\left\langle\mathbf{u}_{0}\right\rangle(\mathbf{x}), \mathbf{u}_{t}^{H}(\mathbf{x}, 0)=\left\langle\mathbf{v}_{0}\right\rangle(\mathbf{x}), \\
\mathbf{w}(\mathbf{x}, \mathbf{y}, 0) & =\mathbf{u}_{0}(\mathbf{x}, \mathbf{y})-\left\langle\mathbf{u}_{0}\right\rangle(\mathbf{x}) \text { and } \mathbf{w}_{t}(\mathbf{x}, \mathbf{y}, 0)=\mathbf{v}(\mathbf{x}, \mathbf{y})-\left\langle\mathbf{v}_{0}\right\rangle
\end{aligned}
$$

may be used to solve for both $\mathbf{u}^{H}$ and $\mathbf{w}$ simultaneously. As discussed earlier, one may utilize the variation of constant formula to decouple the equations for $\mathbf{u}^{H}$ and $\mathbf{w}$. In the presence of nontrivial heterogeneity, that is when $\mathfrak{p}_{\epsilon}, \alpha_{\epsilon}$ and $k_{\epsilon}$ are all nonconstant functions there is the expected history dependence in the final equation for $\mathbf{u}^{H}$.

\subsection{Strong approximation to heterogeneous peridynamics}

In this subsection we give a strong approximation for $\mathbf{u}^{\epsilon}$ using the two scale limit function $\mathbf{u}(\mathbf{x}, \mathbf{y}, t)$ that is uniquely determined by the system. We do this in the event of a smoother initial two scale data. Below is the main theorem we would like to prove.

Theorem 4.10. Suppose that $\mathbf{u}_{0}(\mathbf{x}, \mathbf{y})$, and $\mathbf{v}_{0}(\mathbf{x}, \mathbf{y})$ are in $L^{p}\left(\Omega ; C_{\mathrm{per}}\left(Y ; \mathbb{R}^{d}\right)\right)$, and that $\mathbf{b}(\mathbf{x}, \mathbf{y}, t) \in$ $C\left([0, T] ; L^{p}\left(\Omega ; C_{\mathrm{per}}\left(Y ; \mathbb{R}^{d}\right)\right)\right)$. Suppose also that $\mathbf{u}^{\epsilon}(\mathbf{x}, t)$ is the solution to the peridynamics equation of motion (4.1) with the forcing term $\mathbf{b}^{\epsilon}=\mathbf{b}\left(\mathbf{x}, \frac{\mathbf{x}}{\epsilon}, t\right)$ and the initial data $\mathbf{u}_{0}^{\epsilon}(\mathbf{x})=\mathbf{u}_{0}\left(\mathbf{x}, \frac{\mathbf{x}}{\epsilon}\right)$, and $\mathbf{v}_{0}^{\epsilon}(\mathbf{x})=\mathbf{v}_{0}\left(\mathbf{x}, \frac{\mathbf{x}}{\epsilon}\right)$. Then the unique solution to the nonlocal system of linear equations (4.3)-(4.4) $\mathbf{u}$ is in $C^{2}\left([0, T] ; L^{p}\left(\Omega ; C_{\mathrm{per}}\left(Y ; \mathbb{R}^{d}\right)\right)\right)$. Moreover, for each $t \in[0, T]$,

$$
\lim _{\epsilon \rightarrow 0}\left\|\mathbf{u}^{\epsilon}(\cdot, t)-\mathbf{u}\left(\mathbf{x}, \frac{\mathbf{x}}{\epsilon}, t\right)\right\|_{L^{p}\left(\Omega ; \mathbb{R}^{d}\right)}=0 .
$$


Proof. Given that the two scale initial data $\mathbf{u}_{0}$ and $\mathbf{v}_{0}$ belong to $L^{p}\left(\Omega ; C_{\mathrm{per}}\left(Y ; \mathbb{R}^{d}\right)\right)$, the conclusion that the solution $\mathbf{u}$ to the two scale limit equation is unique and belongs to $C^{2}\left([0, T] ; L^{p}\left(\Omega ; C_{\mathrm{per}}\left(Y ; \mathbb{R}^{d}\right)\right)\right)$ follows from standard semi group theory, provided we can show that $\mathfrak{p}^{-1} \mathcal{L}_{0}$ is a bounded operator on $L^{p}\left(\Omega, C_{\mathrm{per}}\left(Y ; \mathbb{R}^{d}\right)\right.$. The later can be proved using the fact that $\mathfrak{p}, \alpha, k$ and $\beta$ belong to $C_{\mathrm{per}}(Y)$. Indeed, it is not difficult to show from the definition of the operator $\mathcal{L}_{0}$ given in Lemma 4.4 , for any $\mathbf{u} \in L^{p}\left(\Omega ; C_{\mathrm{per}}\left(Y ; \mathbb{R}^{d}\right)\right)$, and any $\mathbf{x} \in \Omega$, $\mathcal{L}_{0} \mathbf{u}(\mathbf{x}, \cdot) \in C_{\text {per }}(Y)$. Moreover, with tedious estimates we can find a positive constant $C$ (that may depend on the bounds of $\mathfrak{p}, \alpha, k$ and also on $\rho_{0}$ and $\left.\rho_{2}\right)$ such that for any $\mathbf{u} \in L^{p}\left(\Omega ; C_{\mathrm{per}}\left(Y ; \mathbb{R}^{d}\right)\right)$,

$$
\left(\int_{\Omega}\left\|\mathcal{L}_{0} \mathbf{u}(\mathbf{x}, \cdot)\right\|_{L^{\infty}(Y)}^{p}\right)^{1 / p} \leq C\left[\|\langle\mathbf{u}\rangle\|_{L^{p}(\Omega)}+\left(\int_{\Omega}\|\mathbf{u}(\mathbf{x}, \cdot)\|_{L^{\infty}(Y)}^{p} \mathrm{~d} \mathbf{x}\right)^{1 / p}\right] .
$$

Noting that for any $\mathbf{u} \in L^{p}\left(\Omega ; C_{\mathrm{per}}\left(Y ; \mathbb{R}^{d}\right)\right)$,

$$
\|\langle\mathbf{u}\rangle\|_{L^{p}(\Omega)} \leq\left(\int_{\Omega}\|\mathbf{u}(\mathbf{x}, \cdot)\|_{L^{\infty}(Y)}^{p} \mathrm{~d} \mathbf{x}\right)^{1 / p}
$$

we have that

$$
\left\|\mathcal{L}_{0} \mathbf{u}\right\|_{L^{p}\left(\Omega ; C_{\mathrm{per}}\left(Y ; \mathbb{R}^{d}\right)\right)} \leq C\|\mathbf{u}\|_{L^{p}\left(\Omega ; C_{\mathrm{per}}\left(Y ; \mathbb{R}^{d}\right)\right)} .
$$

To prove the strong approximation given in (4.9), we follow the argument used in the proof of Theorem 3.3. Denoting the error $\mathbf{e}^{\epsilon}(\mathbf{x}, t)=\mathbf{u}^{\epsilon}(\mathbf{x}, t)-\mathbf{u}\left(\mathbf{x}, \frac{\mathbf{x}}{\epsilon}, t\right)$, we see that it solves the evolution equation

$$
\left\{\begin{array}{l}
\mathbf{e}_{t t}^{\epsilon}(\mathbf{x}, t)=\mathfrak{p}_{\epsilon}^{-1} \mathcal{L}_{\epsilon} \mathbf{e}^{\epsilon}(\mathbf{x}, t)+\mathbf{D}_{\epsilon}(\mathbf{x}, t), \quad \mathbf{x} \in \Omega, t \in[0, T] \\
\mathbf{e}^{\epsilon}(\mathbf{x}, 0)=0 \quad \mathbf{x} \in \Omega \\
\mathbf{e}_{t}^{\epsilon}(\mathbf{x}, 0)=0 \quad \mathbf{x} \in \Omega
\end{array}\right.
$$

where $\mathbf{D}_{\epsilon}(\mathbf{x}, t)=\mathfrak{p}_{\epsilon}^{-1}\left[\mathcal{L}^{\epsilon}\left(\mathbf{u}\left(\mathbf{x}, \frac{\mathbf{x}}{\epsilon}, t\right)-\left(\mathcal{L}_{0} \mathbf{u}\right)\left(\mathbf{x}, \frac{\mathbf{x}}{\epsilon}, t\right)\right]\right.$. If we show that for each $\epsilon>0, \mathbf{D}^{\epsilon}(\mathbf{x}, t) \in$ $C\left([0, T], L^{p}\left(\Omega ; \mathbb{R}^{d}\right)\right)$, we may use $(2.5)$ in Theorem 2.2 to write $\mathbf{e}^{\epsilon}$ in terms of $\mathbf{D}^{\epsilon}$ as

$$
\mathbf{e}^{\epsilon}(t)=\int_{0}^{t} \mathbf{S}\left((t-\tau) \mathcal{P}_{\epsilon}\right) \mathbf{D}^{\epsilon}(\tau) \mathrm{d} \tau
$$

where $\mathcal{P}_{\epsilon}=\mathfrak{p}_{\epsilon}^{-1} \mathcal{L}^{\epsilon}$, and the operator $\mathrm{S}$ is as defined in Section 2. From the above formula, using the same argument as in the proof of Theorem 3.3, we conclude that for each $t,\left\|\mathbf{e}^{\epsilon}\right\|_{L^{p}} \rightarrow 0$, as $\epsilon \rightarrow 0$. The following lemma proofs all assertions in connection with $\mathbf{D}^{\epsilon}$ to complete the proof of the theorem.

Lemma 4.11. For $1<p<\infty$, assume that the two scale vector function $\mathbf{u}(\mathbf{x}, \mathbf{y}, t)$ belongs to $C\left([0, T] ; L^{p}\left(\Omega ; C_{\mathrm{per}}\left(Y ; \mathbb{R}^{d}\right)\right)\right)$. Then

i) for each $\epsilon>0, \mathbf{D}^{\epsilon}(\mathbf{x}, t) \in C\left([0, T], L^{p}\left(\Omega ; \mathbb{R}^{d}\right)\right)$,

ii)

$$
\sup _{\epsilon>0} \sup _{t \in[0, T]}\left\|\mathbf{D}^{\epsilon}(\cdot, t)\right\|_{L^{p}(\Omega)}<\infty, \text { and }
$$

iii) for all $t \in[0, T]$,

$$
\lim _{\epsilon \rightarrow 0}\left\|\mathbf{D}^{\epsilon}(\cdot, t)\right\|_{L^{p}(\Omega)}=0 .
$$

Proof. (Part i) and ii)) Noting the fact that the operators $\mathcal{L}^{\epsilon}$ and $\mathcal{L}_{0}$ are not intrinsically dependent in the time variable and using their uniform boundedness as linear operators, the inequality (4.10) is not difficult to show. To prove part i) we will drop the multiplier $\mathfrak{p}^{-1}$ as it independent of $t$ and is uniformly bounded by $\frac{1}{p_{0}}$. It then 
suffices to show that both $\mathcal{L}^{\epsilon}\left(\mathbf{u}\left(\mathbf{x}, \frac{\mathbf{x}}{\epsilon}, t\right)\right)$ and $\left(\mathcal{L}_{0} \mathbf{u}\right)\left(\mathbf{x}, \frac{\mathbf{x}}{\epsilon}, t\right)$ are in $C\left([0, T] ; L^{p}\left(\Omega ; \mathbb{R}^{d}\right)\right.$. To that end, let us pick $t_{1}, t_{2} \in[0, T]$ and estimate as

$$
\begin{aligned}
\left\|\mathcal{L}^{\epsilon}\left(\mathbf{u}\left(\cdot, \dot{\bar{\epsilon}}, t_{1}\right)\right)-\mathcal{L}^{\epsilon}\left(\mathbf{u}\left(\cdot, \frac{\dot{\bar{\epsilon}}}{\epsilon}, t_{2}\right)\right)\right\|_{L^{p}(\Omega)} & =\left\|\mathcal{L}^{\epsilon}\left(\mathbf{u}\left(\cdot, \frac{\dot{\bar{\epsilon}}}{\epsilon}, t_{1}\right)-\mathbf{u}\left(\cdot, \frac{\dot{\bar{\epsilon}}}{\epsilon}, t_{2}\right)\right)\right\|_{L^{p}(\Omega)} \\
& \leq C\left\|\mathbf{u}\left(\cdot, \frac{\dot{\epsilon}}{\epsilon}, t_{1}\right)-\mathbf{u}\left(\cdot, \dot{-}, t_{2}\right)\right\|_{L^{p}(\Omega)} \\
& \leq C\left\|\mathbf{u}\left(\cdot, \cdot, t_{1}\right)-\mathbf{u}\left(\cdot, \cdot, t_{2}\right)\right\|_{L^{p}(\Omega \times Y)}
\end{aligned}
$$

where we used the uniform boundedness of the operator $\mathcal{L}^{\epsilon}$ in the first inequality and used the property of the space $L^{p}\left(\Omega ; C_{\text {per }}\left(Y ; \mathbb{R}^{d}\right)\right)$ in the second inequality. Now continuity follows from the fact that $\mathbf{u} \in C\left([0, T] ; L^{p}\left(\Omega ; C_{\mathrm{per}}\left(Y ; \mathbb{R}^{d}\right)\right)\right)$.

Next we will work on $\left(\mathcal{L}_{0} \mathbf{u}\right)\left(\mathbf{x}, \frac{\mathbf{x}}{\epsilon}, t\right)$. By definition,

$$
\left(\mathcal{L}_{0} \mathbf{u}\right)\left(\mathbf{x}, \frac{\mathbf{x}}{\epsilon}, t\right)=\mathcal{L}_{\text {ave }}^{1}\langle\mathbf{u}\rangle(\mathbf{x}, t)+\left(\mathcal{L}_{\text {osc }}^{1}\langle\mathbf{u}\rangle\right)\left(\mathbf{x}, \frac{\mathbf{x}}{\epsilon}, t\right)+\mathcal{M}^{1}(\mathbf{u}-\langle\mathbf{u}\rangle)\left(\mathbf{x}, \frac{\mathbf{x}}{\epsilon}, t\right)+\left(\mathcal{M}^{2} \mathbf{u}\right)\left(\mathbf{x}, \frac{\mathbf{x}}{\epsilon}, t\right)
$$

We show that each term in the above expression belongs to the space. For that we merely apply the boundness of the operators together with the following list of elementary facts. Suppose that $\mathbf{u} \in C\left([0, T] ; L^{p}\left(\Omega, C_{\mathrm{per}}\left(Y ; \mathbb{R}^{d}\right)\right)\right)$. Then the following are true.

a) $\langle\mathbf{u}\rangle(\mathbf{x}, t) \in C\left([0, T] ; L^{p}\left(\Omega ; \mathbb{R}^{d}\right)\right)$.

b) $\forall \epsilon>0, \mathbf{u}\left(\mathbf{x}, \frac{\mathbf{x}}{\epsilon}, t\right) \in C\left([0, T] ; L^{p}\left(\Omega ; \mathbb{R}^{d}\right)\right)$, and so are $\mathbb{P}\left(\mathbf{x}, \frac{\mathbf{x}}{\epsilon}\right) \mathbf{u}\left(\mathbf{x}, \frac{\mathbf{x}}{\epsilon}, t\right), \mathbb{P}\left(\mathbf{x}, \frac{\mathbf{x}}{\epsilon}\right)\langle\mathbf{u}\rangle(\mathbf{x}, t)$.

c) $\left(\mathcal{M}^{2} \mathbf{u}\right)\left(\mathbf{x}, \frac{\mathbf{x}}{\epsilon}, t\right) \in C\left([0, T] ; L^{p}\left(\Omega ; \mathbb{R}^{d}\right)\right)$.

Indeed, part a) can be shown as

$$
\left\|\langle\mathbf{u}\rangle\left(\cdot, t_{1}\right)-\langle\mathbf{u}\rangle\left(\cdot, t_{2}\right)\right\|_{L^{p}} \leq \int_{Y}\left\|\mathbf{u}\left(\cdot, \mathbf{y}, t_{1}\right)-\mathbf{u}\left(\cdot, \mathbf{y}, t_{2}\right)\right\|_{L^{p}} \mathrm{~d} \mathbf{y} \leq\left\|\sup _{\mathbf{y} \in Y}\left\{\mathbf{u}\left(\cdot, \mathbf{y}, t_{1}\right)-\mathbf{u}\left(\cdot, \mathbf{y}, t_{2}\right)\right\}\right\|_{L^{p}}
$$

where the first inequality is obtained applying Minkowski's inequality. Part b) follows from the estimate that

$$
\begin{aligned}
\left\|\mathbf{u}\left(\cdot, \frac{\dot{-}}{\epsilon}, t_{1}\right)-\mathbf{u}\left(\cdot, \frac{\dot{-}}{\epsilon}, t_{2}\right)\right\|_{L^{p}(\Omega)} & \leq C\left\|\mathbf{u}\left(\cdot, \cdot, t_{1}\right)-\mathbf{u}\left(\cdot, \cdot, t_{2}\right)\right\|_{L^{p}(\Omega \times Y)} \\
& \leq C\left\|\sup _{\mathbf{y} \in Y}\left\{\mathbf{u}\left(\cdot, \mathbf{y}, t_{1}\right)-\mathbf{u}\left(\cdot, \mathbf{y}, t_{2}\right)\right\}\right\|_{L^{p}} .
\end{aligned}
$$

Part c) follows from the estimate

$$
\left\|\left(\mathcal{M}^{2} \mathbf{u}\right)\left(\cdot, \frac{\dot{\epsilon}}{\epsilon}, t_{1}\right)-\left(\mathcal{M}^{2} \mathbf{u}\right)\left(\cdot, \frac{\dot{-}}{\epsilon}, t_{2}\right)\right\|_{L^{p}} \leq 4\|\alpha\|_{L^{\infty}}\|\beta\|_{L^{\infty}}\left\|\rho_{2}\right\|_{L^{1}}\left\|\sup _{\mathbf{y} \in Y}\left\{\mathbf{u}\left(\cdot, \mathbf{y}, t_{1}\right)-\mathbf{u}\left(\cdot, \mathbf{y}, t_{2}\right)\right\}\right\|_{L^{p}} .
$$

Now, we move to prove the Part iii) of the lemma. Recall for a fixed $t$ and $\mathbf{v}(\mathbf{x})=\mathbf{u}\left(\mathbf{x}, \frac{\mathbf{x}}{\epsilon}, t\right)$,

$$
\begin{aligned}
\mathcal{L}^{\epsilon} \mathbf{v}(\mathbf{x}):= & \int_{\Omega}\left(\alpha_{\epsilon}(\mathbf{x})+\alpha_{\epsilon}\left(\mathbf{x}^{\prime}\right)\right) \frac{\rho_{\epsilon}\left(\mathbf{x}, \mathbf{x}^{\prime}-\mathbf{x}\right)}{\left|\mathbf{x}^{\prime}-\mathbf{x}\right|^{2}}\left(\mathbf{x}^{\prime}-\mathbf{x}\right) \otimes\left(\mathbf{x}^{\prime}-\mathbf{x}\right)\left(\mathbf{v}\left(\mathbf{x}^{\prime}\right)-\mathbf{u}(\mathbf{x})\right) \mathrm{d} \mathbf{x}^{\prime} \\
& +\int_{\Omega} \tau_{\epsilon}\left(\mathbf{x}^{\prime}\right) \boldsymbol{\lambda}_{0}\left(\mathbf{x}^{\prime}-\mathbf{x}\right)\left(\int_{\Omega} \boldsymbol{\lambda}_{1}\left(\mathbf{z}-\mathbf{x}^{\prime}\right) \cdot\left(\mathbf{v}(\mathbf{z})-\mathbf{v}\left(\mathbf{x}^{\prime}\right)\right) \mathrm{d} \mathbf{z}\right) \mathrm{d} \mathbf{x}^{\prime} \\
& +\tau_{\epsilon}(\mathbf{x}) \Lambda(\mathbf{x}) \int_{\Omega} \boldsymbol{\lambda}_{0}(\mathbf{z}-\mathbf{x}) \cdot(\mathbf{v}(\mathbf{z})-\mathbf{v}(\mathbf{x}) \mathrm{d} \mathbf{z}+\mathcal{R}(\mathbf{v}(\mathbf{x}))
\end{aligned}
$$


Moreover,

$$
\begin{aligned}
\left(\mathcal{L}_{0} \mathbf{u}\right)\left(\mathbf{x}, \frac{\mathbf{x}}{\epsilon}, t\right)= & \alpha\left(\frac{\mathbf{x}}{\epsilon}\right) \int_{\Omega} \mathbf{K}_{1}\left(\mathbf{x}^{\prime}-\mathbf{x}\right)\left(\langle\mathbf{u}\rangle\left(\mathbf{x}^{\prime}, t\right)-\mathbf{u}\left(\mathbf{x}, \frac{\mathbf{x}}{\epsilon}, t\right)\right) \mathrm{d} \mathbf{x}^{\prime} \\
& +\int_{\Omega} \mathbf{K}_{1}\left(\mathbf{x}^{\prime}-\mathbf{x}\right)\left(\langle\alpha \mathbf{u}\rangle\left(\mathbf{x}^{\prime}, t\right)-\langle\alpha\rangle \mathbf{u}\left(\mathbf{x}, \frac{\mathbf{x}}{\epsilon}, t\right)\right) \mathrm{d} \mathbf{x}^{\prime} \\
& +\int_{\Omega} \boldsymbol{\lambda}\left(\mathbf{x}^{\prime}-\mathbf{x}\right) \int_{\Omega} \boldsymbol{\lambda}_{0}\left(\mathbf{z}-\mathbf{x}^{\prime}\right)\left[\langle\tau\rangle\left(\mathbf{x}^{\prime}\right)\langle\mathbf{u}\rangle(\mathbf{z}, t)-\langle\tau \mathbf{u}\rangle\left(\mathbf{x}^{\prime}, t\right)\right] \mathrm{d} \mathbf{z} \mathrm{d} \mathbf{x}^{\prime} \\
& +\tau\left(\mathbf{x}, \frac{\mathbf{x}}{\epsilon}\right) \Lambda(\mathbf{x}) \int_{\Omega} \boldsymbol{\lambda}(\mathbf{z}-\mathbf{x})\left(\langle\mathbf{u}\rangle(\mathbf{z}, t)-\mathbf{u}\left(\mathbf{x}, \frac{\mathbf{x}}{\epsilon}, t\right)\right) \mathrm{d} \mathbf{z} \\
& +\int_{B(0, \delta)}\left(\alpha\left(\frac{\mathbf{x}}{\epsilon}\right)+\alpha\left(\frac{\mathbf{x}}{\epsilon}+\mathbf{z}\right)\right) \beta\left(\frac{\mathbf{x}}{\epsilon}, \frac{\mathbf{x}}{\epsilon}+\mathbf{z}\right) \mathbf{K}_{2}(\mathbf{z})\left(\mathbf{u}\left(\mathbf{x}, \frac{\mathbf{x}}{\epsilon}+\mathbf{z}, t\right)-\mathbf{u}\left(\mathbf{x}, \frac{\mathbf{x}}{\epsilon}, t\right)\right) \mathrm{d} \mathbf{z} .
\end{aligned}
$$

The difference $\mathcal{L}^{\epsilon}\left(\mathbf{u}\left(\mathbf{x}, \frac{\mathbf{x}}{\epsilon}, t\right)-\left(\mathcal{L}_{0} \mathbf{u}\right)\left(\mathbf{x}, \frac{\mathbf{x}}{\epsilon}, t\right)\right.$ can be written as

$$
\mathcal{L}^{\epsilon}\left(\mathbf{u}\left(\mathbf{x}, \frac{\mathbf{x}}{\epsilon}, t\right)-\left(\mathcal{L}_{0} \mathbf{u}\right)\left(\mathbf{x}, \frac{\mathbf{x}}{\epsilon}, t\right)=\sum_{k=1}^{8} \mathbf{d}_{k}^{\epsilon}(\mathbf{x}, t)\right.
$$

where

$$
\begin{aligned}
& \mathbf{d}_{1}^{\epsilon}(\mathbf{x}, t)=\alpha_{\epsilon}(\mathbf{x}) \int_{\Omega} \mathbf{K}_{0}\left(\mathbf{x}^{\prime}-\mathbf{x}\right)\left(\mathbf{u}\left(\mathbf{x}^{\prime}, \frac{\mathbf{x}^{\prime}}{\epsilon}, t\right)-\langle\mathbf{u}\rangle\left(\mathbf{x}^{\prime}, t\right)\right) \mathrm{d} \mathbf{x}^{\prime} \\
& \mathbf{d}_{2}^{\epsilon}(\mathbf{x}, t)= \int_{\Omega} \mathbf{K}_{0}\left(\mathbf{x}^{\prime}-\mathbf{x}\right)\left(\alpha_{\epsilon}\left(\mathbf{x}^{\prime}\right) \mathbf{u}\left(\mathbf{x}^{\prime}, \frac{\mathbf{x}^{\prime}}{\epsilon}, t\right)-\langle\alpha \mathbf{u}\rangle\left(\mathbf{x}^{\prime}, t\right)\right) \mathrm{d} \mathbf{x}^{\prime} \\
& \mathbf{d}_{3}^{\epsilon}(\mathbf{x}, t)=\int_{\Omega} \mathbf{K}_{0}\left(\mathbf{x}^{\prime}-\mathbf{x}\right)\left(\alpha_{\epsilon}\left(\mathbf{x}^{\prime}\right)\langle\mathbf{u}\rangle\left(\mathbf{x}^{\prime}, t\right)-\langle\alpha\rangle\langle\mathbf{u}\rangle\left(\mathbf{x}^{\prime}, t\right)\right) \mathrm{d} \mathbf{x}^{\prime} \\
& \mathbf{d}_{4}^{\epsilon}(\mathbf{x}, t)=\int_{\Omega}\left(\langle\alpha\rangle-\alpha_{\epsilon}\left(\mathbf{x}^{\prime}\right)\right) \mathbf{K}_{0}\left(\mathbf{x}^{\prime}-\mathbf{x}\right) \mathbf{u}\left(\mathbf{x}, \frac{\mathbf{x}}{\epsilon}, t\right) \mathrm{d} \mathbf{x}^{\prime} \\
& \mathbf{d}_{5}^{\epsilon}(\mathbf{x}, t)= \int_{\Omega} \tau_{\epsilon}\left(\mathbf{x}^{\prime}\right) \boldsymbol{\lambda}_{0}\left(\mathbf{x}^{\prime}-\mathbf{x}\right)\left(\int_{\Omega} \boldsymbol{\lambda}_{0}\left(\mathbf{z}-\mathbf{x}^{\prime}\right) \cdot\left(\mathbf{u}\left(\mathbf{z}, \frac{\mathbf{z}}{\epsilon}, t\right)-\mathbf{u}\left(\mathbf{x}^{\prime}, \frac{\mathbf{x}^{\prime}}{\epsilon}, t\right)\right) \mathrm{d} \mathbf{z}\right) \mathrm{d} \mathbf{x}^{\prime} \\
&-\int_{\Omega} \boldsymbol{\lambda}_{0}\left(\mathbf{x}^{\prime}-\mathbf{x}\right) \int_{\Omega} \boldsymbol{\lambda}_{0}\left(\mathbf{z}-\mathbf{x}^{\prime}\right)\left[\langle\tau\rangle\left(\mathbf{x}^{\prime}\right)\langle\mathbf{u}\rangle(\mathbf{z}, t)-\langle\tau \mathbf{u}\rangle\left(\mathbf{x}^{\prime}, t\right)\right] \mathrm{d} \mathbf{z} \mathrm{d} \mathbf{x}^{\prime} \\
& \mathbf{d}_{6}^{\epsilon}(\mathbf{x}, t)= \tau_{\epsilon}(\mathbf{x}) \Lambda(\mathbf{x}) \int_{\Omega} \boldsymbol{\lambda}_{0}(\mathbf{z}-\mathbf{x}) \cdot\left(\mathbf{u}\left(\mathbf{z}, \frac{\mathbf{z}}{\epsilon}, t\right)-\langle\mathbf{u}\rangle(\mathbf{z}, t)\right) \mathrm{d} \mathbf{z} \\
& \mathbf{d}_{7}^{\epsilon}(\mathbf{x}, t)= \mathcal{R}\left(\mathbf{u}\left(\mathbf{x}, \frac{\mathbf{x}}{\epsilon}, t\right)\right)
\end{aligned}
$$

and

$$
\begin{aligned}
\mathbf{d}_{8}^{\epsilon}(\mathbf{x}, t)= & \int_{B(0, \delta)}\left[\chi_{\Omega}(\mathbf{x}+\epsilon \mathbf{z})\left(\alpha\left(\frac{\mathbf{x}}{\epsilon}\right)+\alpha\left(\frac{\mathbf{x}}{\epsilon}+\mathbf{z}\right)\right) \beta\left(\frac{\mathbf{x}}{\epsilon}, \frac{\mathbf{x}}{\epsilon}+\mathbf{z}\right)\right. \\
& \left.\times \mathbf{K}_{2}(\mathbf{z})\left(\mathbf{u}\left(\mathbf{x}+\epsilon \mathbf{z}, \frac{\mathbf{x}}{\epsilon}+\mathbf{z}, t\right)-\mathbf{u}\left(\mathbf{x}, \frac{\mathbf{x}}{\epsilon}, t\right)\right)\right] \mathrm{d} \mathbf{z} \\
& -\int_{B(0, \delta)}\left(\alpha\left(\frac{\mathbf{x}}{\epsilon}\right)+\alpha\left(\frac{\mathbf{x}}{\epsilon}+\mathbf{z}\right)\right) \beta\left(\frac{\mathbf{x}}{\epsilon}, \frac{\mathbf{x}}{\epsilon}+\mathbf{z}\right) \mathbf{K}_{2}(\mathbf{z})\left(\mathbf{u}\left(\mathbf{x}, \frac{\mathbf{x}}{\epsilon}+\mathbf{z}, t\right)-\mathbf{u}\left(\mathbf{x}, \frac{\mathbf{x}}{\epsilon}, t\right)\right) \mathrm{d} \mathbf{z}
\end{aligned}
$$

We show that for $i=1, \ldots, 8,\left\|\mathbf{d}_{i}^{\epsilon}(\cdot, t)\right\|_{L^{p}} \rightarrow 0$ as $\epsilon \rightarrow 0$. The strong convergence of $\mathbf{d}_{i}^{\epsilon}(\cdot, t)$ to 0 for $i=1, \ldots, 6$ follows from the fact that the "operators" are of convolution type and applying Lemma 4.5. $\mathbf{d}_{7}^{\epsilon}(\cdot, t)$ can be 
estimated as follows.

$$
\left\|\mathbf{d}_{7}^{\epsilon}(\cdot, t)\right\|_{L^{p}}=\|\mathcal{R}(\mathbf{u}(\mathbf{x}, \mathbf{x} / \epsilon, t))\|_{L^{p}} \leq \epsilon C\|\mathbf{u}(\mathbf{x}, \mathbf{x} / \epsilon, t)\|_{L^{p}} \leq \epsilon C\|\mathbf{u}\|_{L^{p}(\Omega \times Y)} .
$$

To estimate $\mathbf{d}_{8}^{\epsilon}(\cdot, t)$, we denote $\eta\left(\mathbf{y}, \mathbf{y}^{\prime}\right)=\left(\alpha(\mathbf{y})+\alpha\left(\mathbf{y}^{\prime}\right)\right) \beta\left(\mathbf{y}, \mathbf{y}^{\prime}\right)$ and write $\mathbf{d}_{8}^{\epsilon}(\cdot, t)=\mathbf{d}_{8}^{\epsilon, 1}(\cdot, t)+\mathbf{d}_{8}^{\epsilon, 2}(\cdot, t)$ with

$$
\mathbf{d}_{8}^{\epsilon, 1}(\mathbf{x}, t)=\int_{B(0, \delta)} \chi_{\Omega}(\mathbf{x}+\epsilon \mathbf{z}) \eta\left(\frac{\mathbf{x}}{\epsilon}, \frac{\mathbf{x}}{\epsilon}+\mathbf{z}\right) \mathbf{K}_{2}(\mathbf{z})\left(\mathbf{u}\left(\mathbf{x}+\epsilon \mathbf{z}, \frac{\mathbf{x}}{\epsilon}+\mathbf{z}, t\right)-\mathbf{u}\left(\mathbf{x}, \frac{\mathbf{x}}{\epsilon}+\mathbf{z}, t\right)\right) \mathrm{d} \mathbf{z}
$$

and

$$
\mathbf{d}_{8}^{\epsilon, 2}(\mathbf{x}, t)=\int_{B(0, \delta)}\left(\chi_{\Omega}(\mathbf{x}+\epsilon \mathbf{z})-1\right) \eta\left(\frac{\mathbf{x}}{\epsilon}, \frac{\mathbf{x}}{\epsilon}+\mathbf{z}\right) \mathbf{K}_{2}(\mathbf{z})\left(\mathbf{u}\left(\mathbf{x}, \frac{\mathbf{x}}{\epsilon}+\mathbf{z}, t\right)-\mathbf{u}\left(\mathbf{x}, \frac{\mathbf{x}}{\epsilon}, t\right)\right) \mathrm{d} \mathbf{z} .
$$

Let show the strong convergence of each of this terms. Using Minkowski's inequality,

$$
\left\|\mathbf{d}_{8}^{\epsilon, 2}(\cdot, t)\right\|_{L^{p}(\Omega)} \leq C \int_{B(0, \delta)} \rho_{2}(\mathbf{z})\left(\int_{\Omega}\left(\chi_{\Omega}(\mathbf{x}+\epsilon \mathbf{z})-1\right)^{p}\|\mathbf{u}(\mathbf{x}, \cdot)\|_{L^{\infty}(Y)}^{p} \mathrm{~d} \mathbf{x}\right)^{1 / p} \mathrm{~d} \mathbf{z}
$$

For each $\mathbf{z} \in B(0, \delta)$,

$$
\int_{\Omega}\left(\chi_{\Omega}(\mathbf{x}+\epsilon \mathbf{z})-1\right)^{p}\|\mathbf{u}(\mathbf{x}, \cdot)\|_{L^{\infty}(Y)}^{p} \mathrm{~d} \mathbf{x} \rightarrow 0
$$

from which it follows that as $\epsilon \rightarrow 0,\left\|\mathbf{d}_{8}^{\epsilon, 2}(\cdot, t)\right\|_{L^{p}(\Omega)} \rightarrow 0$. Moreover,

$$
\left\|\mathbf{d}_{8}^{\epsilon, 1}(\cdot, t)\right\|_{L^{p}(\Omega)} \leq C \int_{B(0, \delta)} \rho_{2}(\mathbf{z})\left(\int_{\Omega} \chi_{\Omega}(\mathbf{x}+\epsilon \mathbf{z})\|\mathbf{u}(\mathbf{x}+\epsilon \mathbf{z}, \cdot, t)-\mathbf{u}(\mathbf{x}, \cdot, t)\|_{L^{\infty}(Y)}^{p} \mathrm{~d} \mathbf{x}\right)^{1 / p} \mathrm{~d} \mathbf{z}
$$

which, by Lemma 5.2 of [25], goes to 0 , as $\epsilon \rightarrow 0$. For completeness, Lemma 5.2 of [25] states that if $p \in(1, \infty)$, then for any $u \in L^{p}\left(\Omega, C_{\text {per }}\left(Y ; \mathbb{R}^{d}\right)\right)$, we have

$$
\lim _{\|\mathbf{h}\| \rightarrow 0} \int_{\Omega}\|\mathbf{u}(\mathbf{x}+\mathbf{h}, \cdot)-\mathbf{u}(\mathbf{x}, \cdot)\|_{L^{\infty}(Y)}^{p} \mathrm{~d} \mathbf{x}=0 .
$$

\section{Conclusions}

In this work we have studied the homogenized evolution and corrector theory for linear evolution equations associated with integral bounded operators defined over a heterogeneous medium. Our approach uses two-scale operator convergence and captures the oscillations of a periodic medium in the high frequency limit. The ideas have been illustrated in the concrete settings of bounded linear non-local operators described by state based peridynamics and non-local linear convection-diffusion equations. In all settings the solution $\mathbf{u}^{\epsilon}(\mathbf{x}, t)$ of the evolution equation is represented by a strong $L^{p}$ approximation of the form $\mathbf{u}^{H}(\mathbf{x}, t)+\mathbf{w}^{\epsilon}(\mathbf{x}, t)$. Here $\mathbf{u}^{H}$ is the homogenized evolution and the corrector $\mathbf{w}^{\epsilon}$ is described by the coupled evolution $\mathbf{w}(\mathbf{x}, \mathbf{y}, t)$ that directly encodes the effects of local oscillations in the medium. For state based peridynamic evolutions these oscillations are given by the highly oscillatory short range forces and we recover a bottom up multi-scale analysis for the evolution. A similar analysis is carried out for the non-local linear convection-diffusion evolution where the oscillations correspond to highly oscillatory material heterogeneities of the medium. The results derived for the two representative nonlocal continuum models provide good illustration to more general settings and they shed light on how one may improve the effectiveness of nonlocal modeling of multiscale mechanical problems and heterogeneous stochastic processes. We note that the heterogeneities remain when the nonlocal effect diminishes so that the results derived here are not only valid for nonlocal models but are also consistent with the classical homogenization theory for time dependent PDEs in the local limit.

Acknowledgements. The authors would like to thank the referees for their careful reading and their valuable comments that improved the paper. 


\section{REFERENCES}

[1] A. Aguiar and R. Fosdick, A constitutive model for a linearly elastic peridynamic body. Math. Mech. Solids 19 (2013) 502-523.

[2] B. Alali and R. Lipton, Multiscale Analysis of Heterogeneous Media in the Peridynamic formulation. J. Elasticity 106 (2012) 71-103.

[3] G. Allaire, Homogenization and two-scale convergence. SIAM J. Math. Anal. 23 (1992) 1482-1518.

[4] F. Andreu-Vaillo, J.M. Mazen, J.D. Rossi and J.J. Toledo-Melero, Nonlocal Diffusion Problems. In vol. 165 of Math. Surveys Monogr. AMS (2010).

[5] P.W. Bates, P.C. Fife, X. Ren and X. Wang, Traveling waves in a convolution model for phase transitions. Arch. Ration. Mech. Anal. 138 (1997) 105-136.

[6] F. Bobaru and S.A. Silling, Peridynamic 3D problems of nanofiber networks and carbon nanotube-reinforced composites. Materials and Design: Proceedings of Numiform. Amer. Institute Phys. (2004) 1565-1570.

[7] F. Bobaru, S.A. Silling and H. Jiang, Peridynamic fracture and damage modeling of membranes and nanofiber networks. Vol. 5748 of Proc. of the XI International Conference on Fracture. Turin, Italy (2005) 1-6.

[8] H. Brezis, Functional Analysis, Sobolev Spaces and Partial Differential Equations. Springer (2011).

[9] G.W. Clark and R.E. Showalter, Two-scale convergence of a model for flow in a partially fissured medium. Electron. J. Differ. Eq. 1999 (1999) 1-20.

[10] Q. Du, Nonlocal calculus of variations and well-posedness of peridynamics. In Handbook of Peridynamic Modeling, edited by F. Bobaru, J. Foster, P. Geubelle and S. Silling. CRC Press (2016).

[11] Q. Du, M. Gunzburger, R.B. Lehoucq and K. Zhou, Analysis and approximation of nonlocal diffusion problems with volume constraints. SIAM Rev. 54 (2012) 667-696.

[12] Q. Du, M. Gunzburger, R.B. Lehoucq and K. Zhou, Analysis of the volume-constrained peridynamic Navier equation of linear elasticity. J. Elasticity 113 (2013) 193-217.

[13] Q. Du, Z. Huang and R. Lehoucq, Nonlocal convection-diffusion volume-constrained problems and jump processes. Disc. Cont. Dyn. Sys. B 19 (2014) 373-389.

[14] A. Visintin, Towards a two-scale calculus. ESAIM: COCV 12 (2006) 371-397.

[15] E, Weinan, Homogenization of linear and nonlinear transport equations. Comm. Pure Appl. Math. 45 (1992) $301-326$.

[16] E. Emmrich, R.B. Lehoucq and D. Puhst, Peridynamics: A Nonlocal Continuum Theory. Meshfree Methods for Partial Differential Equations VI. Lect. Notes Comput Sci Eng. 89 (2013) 45-65.

[17] K.-J. Engel and R. Nagel, One-Parameter Semigroups for Linear Evolution Equations. Springer Verlag, New York (2000).

[18] W. Gerstle, N. Sau and S.A. Silling, Peridynamic modeling of plain and reinforced concrete structures. SMiRT18: 18th lnt. Conf. Struct. Mech. React. Technol. Beijing (2005).

[19] W. Hu, Y.D. Ha and F. Bobaru, Modeling dynamic fracture and damage in a fiber-reinforced composite lamina with peridynamics. Int. J. Multiscale Comput. Eng. 9 (2011) 707-726.

[20] L. Ignat and J.D. Rossi, A nonlocal convection-diffusion equation. J. Funct. Anal. 251 (2007) 399-437.

[21] B. Kilic, A. Agwai and E. Madenci, Peridynamic theory for progressive damage prediction in center-cracked composite laminates. Compos. Struct. 90 (2009) 141-151.

[22] R.B. Lehoucq and S.A. Silling, Force flux and the peridynamic stress tensor. J. Mech. Phys. Solids 56 (2008) $1566-1577$.

[23] D. Lukkassen, G. Nguetseng and P. Wall, Two-scale convergence. Int. J. Pure Appl. Math. 2 (2002) 35-86.

[24] T. Mengesha and Q. Du, Nonlocal constrained value problems for a linear peridynamic Navier equation. J. Elasticity 116 (2014) 27-51.

[25] T. Mengesha and Q. Du, Multiscale analysis of a linear peridynamics solid. Commun. Math. Sci. 13 (2015) 1193 -1218.

[26] F. Neubrander, Integrated semigroups and their application to the abstract Cauchy problem. Pacific J. Math. 135 (1988) 111-157.

[27] G. Nguetseng, A general convergence result for a functional related to the theory of homogenization. SIAM J. Math. Anal. 20 (1989) 608-623.

[28] A. Pazy, Semigroups of Linear Operators and Applications to Partial Differential Equations. Springer Verlag (1983).

[29] B. Schweizer and Veneroni, Homogenization of plasticity equations with two-scale convergence methods. Appl. Anal. Int. J. 94 (2015) 375-398.

[30] R.E. Showalter, Distributed microstructure models of porous media. Flow in Porous Media: proceedings of the Oberwolfach Conference (1992), edited by U. Hornung. Vol. 114 of International Series of Numerical Mathematics. Birkhauser Verlag Basel (1993) 21-27.

[31] S.A. Silling, Reformulation of elasticity theory for discontinuities and long-range forces. J. Mech. Phys. Solids 48 (2000) 175-209.

[32] S.A. Silling, Linearized theory of peridynamic states. J. Elast. 99 (2010) 85-111.

[33] S.A. Silling, M. Epton, O. Weckner, J. Xu and E. Askari, Peridynamic states and constitutive modeling. J. Elast. 88 (2007) 151-184.

[34] L. Tartar, Memory effects and homogenization. Arch. Rational Mech. Anal. 111 (1990) 121-133. 